CP3-14-17

\title{
Effective field theory approach to top-quark decay at next-to-leading order in QCD
}

\author{
Cen Zhang \\ Centre for Cosmology, Particle Physics and Phenomenology, \\ Université Catholique de Louvain, B-1348 Louvain-la-Neuve, Belgium
}

\begin{abstract}
We present analytical results for top-quark decay processes, in an effective field theory beyond the Standard Model, at next-to-leading order in QCD. We parametrize new physics effects using dimension-six operators, and consider all operators that give rise to non-standard interactions of the top quark. We investigate both the flavor-conserving and flavor-changing decay modes, including their two-body and three-body semi-leptonic final states. The QCD mixing among relevant operators are also taken into account. These results provide all information needed for a complete modelindependent study of top-quark decay at next-to-leading order accuracy, paving the way to global analyses for new physics effects in an effective field theory approach.

PACS numbers: 12.38.Bx,14.65.Ha,14.80.Bn
\end{abstract}

\section{INTRODUCTION}

The discovery of the Higgs boson at the LHC finally completes the Standard Model (SM) [1,2]. The absence of any resonant signal of new physics up to several hundreds of $\mathrm{GeV}$ allows one to parametrize the effects of any possible new physics using an effective field theory (EFT) approach [3-5]. In this approach one assumes that the new physics decouples from the SM in the limit that the energy scale $\Lambda$, which characterizes the new particles and interactions goes to infinity, and at the electroweak scale the deviations from the SM are parameterized by higherdimensional operators that involve only the SM fields. In this paper we also assume that the Higgs boson observed at the LHC is the SM Higgs boson, and that the electroweak $S U(2)_{L} \times U(1)_{Y}$ gauge symmetry is linearly realized.

On the other hand, the top quark continues to be the heaviest particle known, serving as a window to new physics. Measurements on top quark production and decay processes can provide key information on physics beyond the SM. Model independent analyses of the top quark have been perfermed using the EFT approach, see for example Refs. [6-46].

As the precision level of experimental measurements continue to increase, it becomes more and more important to have theoretical predictions at a same or better precision level. As a colored particle, any process involving a top-quark can potentially suffer from a large uncertainty at the leading order (LO) accuracy in QCD. For this reason it is desirable to have predictions in the EFT framework at a next-to-leading order (NLO) accuracy in QCD.

Fortunately, despite being a "non-renormalizable theory", an EFT indeed provides a framework in which radiative corrections can be consistently handeled, see for example Refs. [23, 24, 47-49]. Nevertheless going to the NLO accuracy is not a trivial task, for at least two reasons. First, at the NLO accuracy level more operators start to contribute, and including only a subset of oper- ators is not justified. Second, operators involving quark or gluon fields will in general evolve and mix with each other. As a result extracting information on new physics effects is complicated by the large number of operators. In order to determine or constrain their coefficients, one has to perform a global analysis, taking into account all available data and all relevant operators, a task that, indeed, needs a dedicated effort.

The aim of this paper is to give contribution to this goal, by providing the complete analytical results for topquark decay processes, in the EFT framework at NLO in QCD, including all relevant dimension-six operators. Top-quark decays provide the best places to probe the weak coupling of top quark [50], as well as to discover its flavor-changing neutral couplings [51]. Previous results on QCD corrections to top-quark decays are available in the literature. The $t \rightarrow b W$ decay and $W$-helicity fractions in the SM have been computed at NLO [52] and at NNLO [53]. Decay of a polarized top quark has been considered in Refs. [54, 55]. Ref. [56] has studied the same decay mode but with anomalous $t b W$ couplings. The flavor-changing decays $t \rightarrow u_{i} V$, where $u_{i}$ is $u$ or $c$ and $V$ can be $Z, \gamma$ or $g$, have been studied in Ref. [57$59]$, in terms of dimension-four and five operators (these operators do not have explicit $S U(2)_{L}$ symmetry). The $t \rightarrow u_{i} h$ decay was originally calculated in Ref. [60], and more recently in [42] including the contributions from flavor-changing color-dipole operators.

In this work we complete the analytical results of topquark decay in an EFT framework. We consider the main decay channel $t \rightarrow b W$, the flavor changing decays $t \rightarrow$ $u_{i} V$ and $t \rightarrow u_{i} h$, as well as their corresponding semileptonic three-body final states. Apart from checking existing results, we provide new results, including:

1. The contribution of the top color-dipole operator $O_{t G}$ in the main decay channel.

2. The helicity fraction of $Z$ in $t \rightarrow u_{i} Z$.

3. Differential decay rates for semi-leptonic decays 
(and thus also the finite-width effect of $W$ and $Z$ ), including invariant mass and angular distribution.

4. Contributions from four-fermion operators at NLO.

We also provide the operator mixing at order $\alpha_{s}$.

The paper is organized as follows. In section II we present our formalism and explain the calculation strategies. In section III we summarize the $\mathcal{O}\left(\alpha_{s}\right)$ running and mixing among all relevant operators. We then give our analytical results in section IV. In section $\mathrm{V}$ we discuss some numerical results, and we summarize in section VI.

\section{APPROACH}

\section{A. Operator list}

Assuming electroweak $S U(2)_{L} \times U(1)_{Y}$ gauge symmetry and baryon and lepton number conservation (see Ref. [61] for a discussion about baryon number violation in top quark decay), the higher-dimensional operators least suppressed by inverse powers of $\Lambda$ are the ones with mass dimension six. They are suppressed by $1 / \Lambda^{2}$. In this work we only consider dimension-six operators. The EFT can be written as

$$
\mathcal{L}_{\mathrm{EFT}}=\mathcal{L}_{\mathrm{SM}}+\sum_{i} \frac{C_{i} O_{i}+C_{i}^{*} O_{i}^{\dagger}}{\Lambda^{2}}+\cdots
$$

where $O_{i}$ are the dimension-six operators, and $C_{i}$ are dimensionless coefficients. The ellipsis refers to operators of dimension eight or higher, which we neglect. The complete list of dimension-six operators was first given in Ref. [62], and gradually evolved to an independent operator basis with 59 operators [63]. Here we use the same basis as in Ref. [63]. We will use the following notation for quark fields:

$$
\begin{array}{ll}
Q: & \text { 3rd-generation left-handed quark doublet } \\
q: & \text { 1st-generation left-handed quark doublet } \\
t: & \text { right-handed top quark } \\
b: & \text { right-handed bottom quark } \\
u: & \text { right-handed up and charm quark }
\end{array}
$$

We first list the operators for two-body decay processes. The relevant ones for $t \rightarrow b W$ are flavor diagonal. They are

$$
\begin{aligned}
& O_{\varphi Q}^{(3)}=i \frac{1}{2} y_{t}^{2}\left(\varphi^{\dagger} \overleftrightarrow{D}_{\mu}^{I} \varphi\right)\left(\bar{Q} \gamma^{\mu} \tau^{I} Q\right) \\
& O_{t W}=y_{t} g_{W}\left(\bar{Q} \sigma^{\mu \nu} \tau^{I} t\right) \tilde{\varphi} W_{\mu \nu}^{I} \\
& O_{\varphi \varphi}=i y_{t}^{2}\left(\tilde{\varphi}^{\dagger} D_{\mu} \varphi\right)\left(\bar{t} \gamma^{\mu} b\right) \\
& O_{b W}=y_{t} g_{W}\left(\bar{Q} \sigma^{\mu \nu} \tau^{I} b\right) \varphi W_{\mu \nu}^{I} \\
& O_{t G}=y_{t} g_{s}\left(\bar{Q} \sigma^{\mu \nu} T^{A} t\right) \tilde{\varphi} G_{\mu \nu}^{A} \\
& O_{t \varphi}=-y_{t}^{3}\left(\varphi^{\dagger} \varphi\right)(\bar{Q} t) \varphi
\end{aligned}
$$

where $\tau^{I}$ are the Pauli matrices, $\varphi$ is the Higgs doublet, and $\tilde{\varphi}=i \tau^{2} \varphi^{*}$. The covariant derivative $D_{\mu}$ is defined as

$$
D_{\mu}=\partial_{\mu}-i g_{s} T^{A} G_{\mu}^{A}-i g_{W} \frac{1}{2} \tau^{I} W_{\mu}^{I}-i g_{Y} Y B_{\mu} .
$$

The relevant operators for $t \rightarrow u_{i} V$ and $t \rightarrow u_{i} h$ are flavor off-diagonal. They are

$$
\begin{aligned}
& O_{\varphi q}^{(3,1+3)}=i \frac{1}{2} y_{t}^{2}\left(\varphi^{\dagger} \overleftrightarrow{D}_{\mu}^{I} \varphi\right)\left(\bar{q} \gamma^{\mu} \tau^{I} Q\right) \\
& O_{\varphi q}^{(1,1+3)}=i \frac{1}{2} y_{t}^{2}\left(\varphi^{\dagger} \overleftrightarrow{D}_{\mu} \varphi\right)\left(\bar{q} \gamma^{\mu} Q\right) \\
& O_{\varphi u}^{(1+3)}=i \frac{1}{2} y_{t}^{2}\left(\varphi^{\dagger} \overleftrightarrow{D}_{\mu} \varphi\right)\left(\bar{u} \gamma^{\mu} t\right) \\
& O_{u B}^{(13)}=y_{t} g_{Y}\left(\bar{q} \sigma^{\mu \nu} t\right) \tilde{\varphi} B_{\mu \nu} \\
& O_{u W}^{(13)}=y_{t} g_{W}\left(\bar{q} \sigma^{\mu \nu} \tau^{I} t\right) \tilde{\varphi} W_{\mu \nu}^{I} \\
& O_{u G}^{(13)}=y_{t} g_{s}\left(\bar{q} \sigma^{\mu \nu} T^{A} t\right) \tilde{\varphi} G_{\mu \nu}^{A} \\
& O_{u \varphi}^{(13)}=-y_{t}^{3}\left(\varphi^{\dagger} \varphi\right)(\bar{q} t) \tilde{\varphi}
\end{aligned}
$$

where superscript $(1+3)$ and $(13)$ denotes the flavor structure. For operators with (13) superscript [Eqs. (12)(15)], a similar set of operators with (31) flavor structure can be obtained by interchanging (13) $\leftrightarrow(31), t \leftrightarrow u$ and $Q \leftrightarrow q$. The Hermitian conjugation of these (31) operators will contribute to $t \rightarrow u_{i} V$ or $t \rightarrow u_{i} h$ in a similar way, but with chirality structures opposite to those from the (13) operators. On the other hand, for the operators with superscript $(1+3)$ superscript [Eqs. (9)-(11)], interchanging the first and the third generation simply gives the Hermitian conjugation of themselves, therefore needs not to be considered separately. Replacing the up quark field with the charm quark field will give the same set of operators for $(2+3),(23)$ and (32) flavor structures.

We have normalized these operators by adding factors of $y_{t}, g_{W}, g_{Y}$ and $g_{s}$. Here $y_{t}$ is the top-quark Yukawa coupling, $g_{W}$ is the weak gauge coupling, $g_{Y}$ is the hypercharge gauge coupling, and $g_{s}$ is the strong coupling. More specifically, we attach a $y_{t}$ for each Higgs field, a $g_{W}\left(g_{Y}\right)$ for each $W(B)$ field, and a $g_{s}$ for each gluon field. This is helpful in determining the order of operator mixing. In general, the order of mixing between two operators depends on the normalization factors of both operators, and so its definition itself has some ambiguity. In this work we are interested in NLO effects in QCD, and we consider them as any corrections coming from a virtual gluon in a loop or a real gluon emission (and gluon splitting in $t \rightarrow u_{i} g$ ). With the above normalization factors, these corrections are automatically of order $\mathcal{O}\left(\alpha_{s}\right)$, and thus it is convenient to present results using these conventions. These normalization factors are also consistent with the naive dimension analysis [64], and the presented operators are constructed in the following form:

$$
f^{2} \Lambda^{2}\left(\frac{\psi}{f \sqrt{\Lambda}}\right)^{2}\left(\frac{y_{t} h}{\Lambda}\right)^{a}\left(\frac{D}{\Lambda}\right)^{b}\left(\frac{g X}{\Lambda^{2}}\right)^{c}
$$


with $\Lambda \sim 4 \pi f$ and $X$ represents any gauge-field strength tensor. It has been shown in Ref. [65] that with this convention the anomalous dimension for the operator coefficients depends on products of powers of $\lambda /(4 \pi)^{4}$, $g^{2} /(4 \pi)^{2}$, and $y^{2} /(4 \pi)^{2}$ as expected. In this work we are interested in the $g_{s}^{2} /(4 \pi)^{2}$ part, which is related to a virtual or real gluon correction, and is expected to be important in top-quark related processes. The $y_{t}$ involved in the normalization is defined by the on-shell top-quark mass:

$$
y_{t}=\frac{\sqrt{2} m_{t}}{v},
$$

where $v$ is the vacuum expectation value of the Higgs field. This is just for simplicity. As a result, it does not contribute to the anomalous dimension of the operators at order $\alpha_{s}$.

Following Refs. [63, 66], we have defined the Hermitian derivative terms:

$$
\begin{aligned}
& \left(\varphi^{\dagger} \overleftrightarrow{D}_{\mu} \varphi\right) \equiv i \varphi^{\dagger}\left(D_{\mu}-\overleftarrow{D}_{\mu}\right) \varphi \\
& \left(\varphi^{\dagger} \overleftrightarrow{D}_{\mu}^{I} \varphi\right) \equiv i \varphi^{\dagger}\left(\tau^{I} D_{\mu}-\overleftarrow{D}_{\mu} \tau^{I}\right) \varphi
\end{aligned}
$$

A relative plus sign between the two terms on the r.h.s. would give rise to redundant operators (in the sense that they can be reduced to other operators like $O_{t \varphi}$ or $O_{u \varphi}^{(13)}$ by equations of motion). The advantage of using these definitions is that these terms do not involve a Higgs field, therefore the number of relevant operators in $t \rightarrow u_{i} h$ is reduced. The flavor diagonal operator $O_{\varphi Q}^{(3)}$ defined in this way is Hermitian, so we can ignore the imaginary part of its coefficient. Also note that we always add a Hermitian conjugation of an operator to the Lagrangian, even if the operator is Hermitian by itself.

The operator $O_{t \varphi}$ does not affect the $t \rightarrow b W$ decay. We include it here only because in principle this operator is required as a counterterm to render the top-leg correction from $O_{t G}$ finite. In practice this can be avoided, as we will discuss in Section II C. Similarly, the operator $O_{u \varphi}^{(13,31)}$ is required as a counterterm to regulate the oneloop $\bar{u} t$ mixing from $O_{u G}^{(13,31)}$ and from itself. They are important in $t \rightarrow u_{i} h$ decay, but can be safely ignored in $t \rightarrow u_{i} V$, as has been done in previous calculations.

We now list the four-fermion operators, relevant in semi-leptonic decays [9]. These operators have two quark fields and two lepton fields. We again choose the operator basis as presented in Ref. [63], where these operators are Fierzed into the form of a quark current times a lepton current. This has the advantage that the QCD correction can be factorized, and for vector and scalar current it is essentially the same as in the two-body decays, for a given invariant mass of the lepton pair. This allows us to infer some results from two-body decays. In addition, these operators do not mix at order $\alpha_{s}$, because QCD correction does not affect the lepton current.

We divide the four-fermion operators into three classes, according to their Lorentz structures: the vector-vector operator $(\mathrm{V}-\mathrm{V})$, the scalar-scalar operator $(\mathrm{S}-\mathrm{S})$, and the tensor-tensor operator (T-T). The operators that contribute to flavor-conserving decay $t \rightarrow b \nu e^{+}$are: $\mathrm{V}-\mathrm{V}$

$$
O_{l Q}^{(3)}=\left(\bar{l} \gamma_{\mu} \tau^{I} l\right)\left(\bar{Q} \gamma^{\mu} \tau^{I} Q\right)
$$

S-S

$$
\begin{aligned}
& O_{l e b Q}=(\bar{l} e)(\bar{b} Q) \\
& O_{l e Q t}^{(1)}=(\bar{l} e) \varepsilon(\bar{Q} t)
\end{aligned}
$$

$\mathrm{T}-\mathrm{T}$

$$
O_{l e Q t}^{(3)}=\left(\bar{l} \sigma_{\mu \nu} e\right) \varepsilon\left(\bar{Q} \sigma^{\mu \nu} t\right)
$$

where $\varepsilon=i \tau^{2}$. The following ones contribute to FCNC decay, $t \rightarrow u l^{+} l^{-}$:

$\mathrm{V}-\mathrm{V}$

$$
\begin{aligned}
& O_{l q}^{(1,1+3)}=\left(\bar{l} \gamma_{\mu} l\right)\left(\bar{q} \gamma^{\mu} Q\right) \\
& O_{l q}^{(3,1+3)}=\left(\bar{l} \gamma_{\mu} \tau^{I} l\right)\left(\bar{q} \gamma^{\mu} \tau^{I} Q\right) \\
& O_{l u}^{(1+3)}=\left(\bar{l} \gamma_{\mu} l\right)\left(\bar{u} \gamma^{\mu} t\right) \\
& O_{q e}^{(1+3)}=\left(\bar{q} \gamma_{\mu} Q\right)\left(\bar{e} \gamma^{\mu} e\right) \\
& O_{e u}^{(1+3)}=\left(\bar{e} \gamma_{\mu} e\right)\left(\bar{u} \gamma^{\mu} t\right)
\end{aligned}
$$

S-S

$$
\begin{aligned}
& O_{l e q u}^{(1,13)}=(\bar{l} e) \varepsilon(\bar{q} t) \\
& O_{l e q u}^{(1,31)}=(\bar{l} e) \varepsilon(\bar{Q} u)
\end{aligned}
$$

$\mathrm{T}-\mathrm{T}$

$$
\begin{aligned}
& O_{l e q u}^{(3,13)}=\left(\bar{l} \sigma_{\mu \nu} e\right) \varepsilon\left(\bar{q} \sigma^{\mu \nu} t\right) \\
& O_{l e q u}^{(3,31)}=\left(\bar{l} \sigma_{\mu \nu} e\right) \varepsilon\left(\bar{Q} \sigma^{\mu \nu} u\right) .
\end{aligned}
$$

\section{B. Calculation strategy}

In this work we compute the top decay processes at NLO in QCD but at LO in $C / \Lambda^{2}$. Calculation of higher orders in $C / \Lambda^{2}$ requires a complete knowledge of dimension-eight operators, and is beyond the scope of this paper. For the FCNC decays, LO in $C / \Lambda^{2}$ means order $\left(C / \Lambda^{2}\right)^{2}$ since there is not SM contribution. For $t \rightarrow b W$, with left-handed or longitudinal polarization of $W$, the LO result is of order $C / \Lambda^{2}$. The right-handed helicity vanishes at the tree level in the zero bottom-quark mass limit, and LO contributions may come from order $\left(C / \Lambda^{2}\right)^{2}, \alpha_{s} C / \Lambda^{2}$, or $C m_{b} / \Lambda^{2} m_{t}$. The non-zero bottomquark mass effect at tree level in $t \rightarrow b W$ has been given in Ref. [50]. We will take this effect into account when presenting numerical results, but we will not show the analytical expressions.

For $t \rightarrow b W$ and $t \rightarrow u_{i} Z$ we compute three helicity states separately for $W$ and $Z$. This is done 
by using the helicity projection operators described in Ref. [52]. The helicity fractions can then be used to derive the differential decay rate of $t \rightarrow b W^{*} \rightarrow b \nu e^{+}$and $t \rightarrow u Z^{*}, u \gamma^{*} \rightarrow u l^{+} l^{-}$. By considering three-body final states we are also including the off-shellness and the finite width effects from $W$ and $Z$. For three-body decays mediated by four-fermion S-S and V-V operators, the differential decay rates can be inferred from two-body results at the NLO level. For T-T operators (and their interference with S-S operators), we use a different set of projection operators to decompose the tensor interaction. We discuss this at the end of Section II D.

We use dimensional regularization [67] to regulate both UV and IR divergences. Whenever $\gamma^{5}$ is present in our computation, we use the following prescription based on the 't Hooft-Veltman scheme [68, 69]:

$$
\begin{aligned}
& \gamma^{5} \rightarrow\left(1-8 a_{s}\right) \frac{i}{4} \varepsilon_{\nu_{1} \nu_{2} \nu_{3} \nu_{4}} \gamma^{\nu_{1}} \gamma^{\nu_{2}} \gamma^{\nu_{3}} \gamma^{\nu_{4}} \\
& \gamma_{\mu} \gamma^{5} \rightarrow\left(1-4 a_{s}\right) \frac{i}{3 !} \epsilon_{\mu \nu_{1} \nu_{2} \nu_{3}} \gamma^{\nu_{1}} \gamma^{\nu_{2}} \gamma^{\nu_{3}} \\
& \sigma_{\mu \nu} \gamma^{5} \rightarrow-\frac{i}{2} \epsilon_{\mu \nu \alpha \beta} \sigma^{\alpha \beta},
\end{aligned}
$$

where $a_{s}=C_{F} \alpha_{s} /(4 \pi)$.

The calculation procedure for each process can be divided into two steps:

1. UV-divergent part, which gives rise to operator mixing and RG equations.

2. UV-finite part, which gives the finite part of the matrix element.

In the first step, we calculate the UV-divergent part arising from the loop diagrams, and identify the UV counterterms by applying the $\overline{M S}$ scheme and requiring that the UV-divergent terms in the matrix element cancel. The outcome of this procedure is a set of counterterms for dimension-six operators. We then proceed to work out the $\mathcal{O}\left(\alpha_{s}\right)$ anomalous dimension and the $\mathrm{RG}$ equations of the operator coefficients. These equations characterize the $\mathcal{O}\left(\alpha_{s}\right)$ running and mixing of the coefficients and can be used to evolve them from a higher scale down to the scale of the top quark mass. We summarize them in Section III.

In the second step we calculate the UV-finite part. The IR-divergences are canceled by including the real gluon emission (and for $t \rightarrow u_{i} g$ also the gluon splitting) corrections. The final result is given in terms of the coefficients of these operators defined at the scale of the top quark mass. We present these results in Section IV.

For the flavor-changing operators, we will only consider the $(1+3),(13)$ and (31) structure, keeping in mind that similar results for $t \rightarrow c V$ and $t \rightarrow c h$ can be obtained by replacing $C_{i}^{(1+3),(13),(31)} \rightarrow C_{i}^{(2+3),(23),(32)}$.

Throughout this paper, we ignore the bottom quark and other light quark masses, and assume that the CKM matrix is identity.

\section{Operator renormalization}

We first discuss a few issues in the operator renormalization procedure. For the SM we use a scheme which subtracts the massless modes according to $\overline{M S}$, and the massive ones at zero momentum [70]. For light quark and gluon we use:

$\delta Z_{2}^{(q)}=-\frac{\alpha_{s}}{3 \pi} D_{\epsilon}\left(\frac{1}{\epsilon_{U V}}-\frac{1}{\epsilon_{I R}}\right)$
$\delta Z_{2}^{(g)}=-\frac{\alpha_{s}}{2 \pi} D_{\epsilon}\left(\frac{N_{f}}{3}-\frac{5}{2}\right)\left(\frac{1}{\varepsilon_{U V}}-\frac{1}{\varepsilon_{I R}}\right)-\frac{\alpha_{s}}{6 \pi} D_{\epsilon} \frac{1}{\epsilon_{U V}}$,

and for the strong coupling:

$\delta Z_{g_{s}}=\frac{\alpha_{s}}{4 \pi} \Gamma(1+\epsilon)(4 \pi)^{\epsilon}\left(\frac{N_{f}}{3}-\frac{11}{2}\right) \frac{1}{\epsilon_{U V}}+\frac{\alpha_{s}}{12 \pi} D_{\epsilon} \frac{1}{\epsilon_{U V}}$

where

$$
D_{\epsilon} \equiv \Gamma(1+\epsilon)\left(\frac{4 \pi \mu^{2}}{m_{t}^{2}}\right)^{\epsilon}
$$

We consider five light flavors in the running of $\alpha_{s}$. We then apply the $\overline{M S}$ scheme to the dimension-six operators. The operators considered here will only mix with other dimension-six operators, and the counterterms are given by

$$
C_{i}^{0} \rightarrow Z_{i, j} C_{j}=(\mathbf{1}+\delta Z)_{i, j} C_{j}
$$

In general they could also affect the running of SM parameters [65], but this is not an $\mathcal{O}\left(\alpha_{s}\right)$ effect and is irrelevant for this study.

Special care needs to be taken for the renormalization of top-quark field. This is because the operator $O_{t G}$ contribute to the top quark self energy through the diagrams in Figure 1. If one follows the standard on-shell renormalization condition, the divergence in the mass term will require a mass counterterm from the operator $O_{t \varphi}$. On the other hand, the wavefunction part needs to be canceled by other operators that are redundant in our operator basis. This makes the problem more complicated because these redundant operators will also contribute to $t \rightarrow b W$. Furthermore, since we use $\overline{M S}$ scheme for the operators, only the pole is canceled in leg corrections, and the remaining finite part needs to be taken into account.
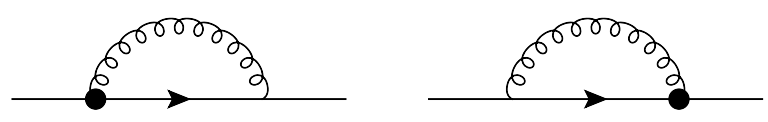

FIG. 1: Top-quark self-energy correction from $O_{t G}$.

The easiest way to avoid the complication, is to replace the $\varphi^{\dagger} \varphi$ part in all operators by $\left(\varphi^{\dagger} \varphi-v^{2} / 2\right)$. This removes the dimension-four terms in a dimension-six operator. It corresponds to a redefinition of a SM term, 
and thus has no physical effects. The only differences are that some vertices involving Higgs field will be shifted, and that the two-point counterterms are provided only by SM terms. Therefore one can adjust the SM counterterms to have on-shell subtraction including dimension-six terms. The dimension-six counterterms will then be fixed only by requiring that the matrix element is finite.

If we assume no $C P$-violation, then the coefficient $C_{t G}$ is real. Following the above strategy we obtain the following counterterms for the top quark:

$$
\begin{aligned}
\delta Z_{2}^{(t)}= & -\frac{\alpha_{s}}{3 \pi} D_{\epsilon}\left(\frac{1}{\varepsilon_{U V}}+\frac{2}{\varepsilon_{I R}}+4\right) \\
& +\operatorname{Re} C_{t G} \frac{m_{t}^{2}}{\Lambda^{2}}\left(-\frac{2 \alpha_{s}}{\pi}\right) D_{\epsilon}\left(\frac{1}{\varepsilon_{U V}}+\frac{1}{3}\right) \\
\frac{\delta m_{t}}{m_{t}}= & -\frac{\alpha_{s}}{3 \pi} D_{\epsilon}\left(\frac{3}{\varepsilon_{U V}}+4\right) \\
& +\operatorname{Re} C_{t G} \frac{m_{t}^{2}}{\Lambda^{2}}\left(-\frac{4 \alpha_{s}}{\pi}\right) D_{\epsilon}\left(\frac{1}{\varepsilon_{U V}}+\frac{1}{3}\right) .
\end{aligned}
$$

These results would apply even if $C_{t G}$ is complex. In this case there will be terms proportional to $i \gamma^{5}$ left in the top-quark self-energy correction. They only give a phase to the amplitude of $t \rightarrow b W$, which will not interfere with the SM amplitude, and so these terms have no effect at $\mathcal{O}\left(\alpha_{s}\right)$

We use the same strategy to deal with the two-point $u-t$ function arising at one-loop, from the operator $O_{u G}^{(13,31)}$. We redefine the operator $O_{u \varphi}^{(13)}$ by

$$
O_{u \varphi}^{(13)}=-y_{t}^{3}\left(\varphi^{\dagger} \varphi-\frac{v^{2}}{2}\right)(\bar{q} t) \tilde{\varphi}
$$

and in a similar way for $O_{u \varphi}^{(31)}$. Note the $u t h$ vertex is rescaled by a factor of $2 / 3$. We then introduce the following $u-t$ counterterms:

$$
\begin{aligned}
u_{L, 0} & =\left(1+\frac{1}{2} \delta Z_{2}^{(q)}\right) u_{L}+\frac{1}{2} \delta Z_{u t}^{L} t_{L} \\
u_{R, 0} & =\left(1+\frac{1}{2} \delta Z_{2}^{(q)}\right) u_{R}+\frac{1}{2} \delta Z_{u t}^{R} t_{R} \\
t_{L, 0} & =\left(1+\frac{1}{2} \delta Z_{2}^{(t)}\right) t_{L}+\frac{1}{2} \delta Z_{u t}^{L} u_{L} \\
t_{R, 0} & =\left(1+\frac{1}{2} \delta Z_{2}^{(t)}\right) t_{R}+\frac{1}{2} \delta Z_{u t}^{R} u_{R}
\end{aligned}
$$

with

$$
\begin{aligned}
& \frac{1}{2} \delta Z_{u t}^{L}=\frac{\alpha_{s}}{\pi} \frac{C_{u G}^{(13)} m_{t}^{2}}{\Lambda^{2}} D_{\epsilon}\left(\frac{1}{\varepsilon_{U V}}-\frac{2}{3}-i \pi\right) \\
& \frac{1}{2} \delta Z_{t u}^{L *}=-\frac{2 \alpha_{s}}{\pi} \frac{C_{u G}^{(13)} m_{t}^{2}}{\Lambda^{2}} D_{\epsilon}\left(\frac{1}{\varepsilon}+\frac{1}{3}\right) \\
& \frac{1}{2} \delta Z_{u t}^{R}=\frac{\alpha_{s}}{\pi} \frac{C_{u G}^{(31) *} m_{t}^{2}}{\Lambda^{2}} D_{\epsilon}\left(\frac{1}{\varepsilon}-\frac{2}{3}-i \pi\right) \\
& \frac{1}{2} \delta Z_{t u}^{R *}=-\frac{2 \alpha_{s}}{\pi} \frac{C_{u G}^{(31) *} m_{t}^{2}}{\Lambda^{2}} D_{\epsilon}\left(\frac{1}{\varepsilon}+\frac{1}{3}\right) .
\end{aligned}
$$

These counterterms are adjusted such that the two-point $u-t$ function is exactly canceled both at the up-quark shell and the top-quark shell. This is convenient because one does not need to include any leg correction diagrams in flavor-changing processes.

\section{Three-body final states}

We briefly outline our approach for three-body decays, i.e. $t \rightarrow b \nu e^{+}$and $t \rightarrow u l^{+} l^{-}$. We consider two cases. The first is that a two-fermion operator contribute to a two-body decay $t \rightarrow q+X$, and the latter is followed by $X \rightarrow l^{+} l^{-}$. The second case is that a direct contribution comes from a contact tqll interaction.

The contributions from two-fermion operators can be completely factorized for a given invariant mass of lepton pair, therefore one can make use of the NLO two-body decay results to infer the three-body decay rates. The same approach is often used in $t$ and $B$ decays, nevertheless we sketch out the steps here, so that we can easily include any non-standard interactions and four-fermion operators later on.

Consider $t \rightarrow q+W^{*}$ followed by $W^{*} \rightarrow e^{+} \nu$ as an example. Here $W^{*}$ is an off-shell $W$ with virtuality $Q^{2}$. The amplitude of the three-body final state can be obtained from the two-body ones simply by replacing the $W$ polarization vector with a lepton current

$$
\begin{aligned}
& M_{\mu}^{(b W)}\left(m_{W}^{2}\right) \epsilon^{\mu *} \\
& \rightarrow M_{\mu}^{(b W)}\left(Q^{2}\right) \frac{g}{\sqrt{2}} \bar{\nu}\left(k_{1}\right) \gamma^{\mu} P_{L} e\left(k_{2}\right) D\left(Q^{2}, m_{W}, \Gamma_{W}\right)^{-1}
\end{aligned}
$$

where $k_{1}$ and $k_{2}$ are the momenta of final state leptons, $M_{\mu}^{(b W)}\left(Q^{2}\right)$ is the two-body amplitude with the $W$ mass replaced by the virtuality of the off-shell $W$, and

$$
D\left(Q^{2}, m_{W}, \Gamma_{W}\right)=Q^{2}-m_{W}^{2}+i m_{W} \Gamma_{W} .
$$

Note this procedure can be applied both at LO and NLO in QCD, as the QCD corrections do not affect lepton current. $\Gamma_{W}$ can be a function of $Q^{2}$.

The resulting three-body amplitude can further be decomposed with the three polarization vectors of the virtual $W$ :

$$
\begin{aligned}
M^{\left(b e^{+} \nu\right)}= & -\sum_{i=+, 0,-} M_{\mu}^{(b W)}\left(Q^{2}\right) \epsilon_{i}^{\mu *} \epsilon_{i}^{\nu} \\
& \times \frac{g}{\sqrt{2}} \bar{\nu}\left(k_{1}\right) \gamma_{\nu} P_{L} e\left(k_{2}\right) D\left(Q^{2}, m_{W}, \Gamma_{W}\right)^{-1} \\
\equiv & -\sum_{i=+, 0,-} M_{i}^{(b W)}\left(Q^{2}\right) \frac{g}{\sqrt{2}} L_{i}^{L} D\left(Q^{2}, m_{W}, \Gamma_{W}\right)^{-1}
\end{aligned}
$$

where $M_{i}^{(b W)}$ is the two-body amplitude with a specific polarization $i$, and $L_{i}^{L}$ denotes the final state left-handed lepton current polarized to the same state. We use $(\theta, \phi)$ 
to denote the direction of the $e^{+}$three-momentum in the $W$ rest frame, relative to the $W$ momentum in the topquark rest frame. Then one can square the amplitude and integrate over the $\phi$ angle:

$$
\begin{aligned}
\int \frac{\mathrm{d} \phi}{4 \pi}\left|M^{b e^{+} \nu}\right|^{2} & =\left(M_{i}^{(b W)}\left(Q^{2}\right) M_{j}^{*(b W)}\left(Q^{2}\right)\right) \\
& \times \frac{g^{2}}{2}\left|D\left(Q^{2}, m_{W}, \Gamma_{W}\right)\right|^{-2} \int \frac{\mathrm{d} \phi}{4 \pi}\left(L_{i}^{L} L_{j}^{L *}\right) .
\end{aligned}
$$

Only the diagonal terms $(i=j)$ in $L_{i}^{L} L_{j}^{L *}$ survive after integration:

$$
\int \frac{\mathrm{d} \phi}{4 \pi}\left(L_{i}^{L} L_{j}^{L *}\right)=Q^{2} \delta_{i j} f_{i}(\cos \theta)
$$

where

$$
\begin{aligned}
& f_{+}(\cos \theta)=\frac{1}{4}(1+\cos \theta)^{2} \\
& f_{0}(\cos \theta)=\frac{1}{2} \sin ^{2} \theta \\
& f_{-}(\cos \theta)=\frac{1}{4}(1-\cos \theta)^{2},
\end{aligned}
$$

so in the end one only needs $\left|M_{i}^{(b W)}\left(Q^{2}\right)\right|^{2}$, i.e. the twobody squared amplitude for a given $W^{*}$ helicity.

Next we consider phase space. One can always write a $n$-body phase space as a $(n-1)$ - body phase space times a two-body phase space. For $n=3$,

$$
\begin{aligned}
\frac{\mathrm{d} \Phi^{\left(b e^{+} \nu\right)}}{\mathrm{d} Q^{2}} & =\frac{1}{2 \pi} \Phi^{(b W)}\left(Q^{2}\right) \times \Phi^{\left(e^{+} \nu\right)} \\
& =\frac{1}{16 \pi^{2}} \Phi^{(b W)}\left(Q^{2}\right) \int \mathrm{d} \cos \theta \int \frac{d \phi}{4 \pi} .
\end{aligned}
$$

The same can be done for $n=4$ i.e. $b e^{+} \nu g$ final state, so the real corrections can be taken into account in the same way. Putting all pieces together, we have the result for $t \rightarrow b e^{+} \nu$ at NLO in terms of $t \rightarrow b W$ partial widths:

$$
\begin{aligned}
& \frac{\mathrm{d} \Gamma_{b e^{+} \nu}}{\mathrm{d} Q^{2} \mathrm{~d} \cos \theta}=\frac{Q^{2}}{16 \pi^{2}} \frac{g^{2}}{2}\left|D\left(Q^{2}, m_{W}, \Gamma_{W}\right)\right|^{-2} \times \\
& {\left[\Gamma_{b W}^{(+)} f_{+}(\cos \theta)+\Gamma_{b W}^{(0)} f_{0}(\cos \theta)+\Gamma_{b W}^{(-)} f_{-}(\cos \theta)\right] .}
\end{aligned}
$$

In the case of $t \rightarrow u l^{+} l^{-}$the situation is more complicated, because of the interference between $t \rightarrow u Z^{*}$ and $t \rightarrow u \gamma^{*}$ with the same semi-leptonic final state. We leave the details to Section IV, where the analytical results will be given.

The contributions from S-S and V-V types of fourfermion operators can be incorporated in a same way. In each case, the amplitude from these four-fermion operators mimics the amplitude of $t \rightarrow q X^{*} \rightarrow q l^{+} l^{-}$, at both LO and NLO, up to an overall factor and an $X$ propagator, where $X$ can be a scalar or a vector. Since the propagator is a pure number at any given $Q^{2}$, the problem can be converted to the NLO correction to twobody decays, and previous results can be used, with some operator coefficients shifted to include the effect of fourfermion operators. ${ }^{1}$ In this way the interference between two-body decays and four-fermion operators is automatically included.

For T-T type of four-fermion operators, and their interference with S-S operators, the results cannot be inferred from two-body decays, but the differential decay rates can be computed in a similar way, using a set of projection operators to decompose the tensor interaction and to convert the problem to a two-body decay:

$$
P_{i, j}^{\mu \nu \mu^{\prime} \nu^{\prime}}(p, k)=\epsilon_{i}^{\mu \nu}(p, k) \epsilon_{j}^{\mu^{\prime} \nu^{\prime}}(p, k)
$$

where $p$ is the top-quark momentum, $k$ is the momentum of the lepton-pair system, and $\epsilon_{i}^{\mu \nu}$ is a complete basis for antisymmetric tensors:

$$
\sum_{i} \epsilon_{i}^{\mu \nu} \epsilon_{i}^{\mu^{\prime} \nu^{\prime}}=\frac{1}{2}\left(g^{\mu \mu^{\prime}} g^{\nu \nu^{\prime}}-g^{\mu \nu^{\prime}} g^{\nu \mu^{\prime}}\right) .
$$

$\epsilon_{i}^{\mu \nu}$ can be constructed from $k^{\mu}$ and its three polarization vectors $\epsilon_{i}^{\mu}(k)$. This effectively allows us to convert the problem to a two-body decay $t \rightarrow q+X^{*}$, where $X$ is some fictitious tensor particle which doesn't propagate, similar to what has been done for $t \rightarrow b W^{*} \rightarrow b e^{+} \nu$. The angular distribution of the lepton-pair can then be derived from the polarization state of $X$. The derivation of $P_{i, j}^{\mu \nu \mu^{\prime} \nu^{\prime}}$ is straightforward, but the results are tedious, so we will not display them here.

Finally, there is no interference between V-V and S-S or $\mathrm{V}-\mathrm{V}$ and $\mathrm{T}-\mathrm{T}$ operators, due to chirality suppression of the leptons.

\section{OPERATOR MIXINGS}

In this section we summarize the operator running and mixing at $\mathcal{O}\left(\alpha_{s}\right)$ that are relevant in our analysis. We will also extend a bit and include all two-quark operators that involve a third-generation quark. A complete calculation of the anomalous dimension matrix for all dimension-six operators can be found in Ref. [65, 71, 72]. Our results are in agreement with the $\mathcal{O}\left(\alpha_{s}\right)$ terms in the complete results.

The anomalous dimension matrix $\gamma$ is defined such that

$$
\mu \frac{\mathrm{d} C_{i}(\mu)}{\mathrm{d} \mu}=\gamma_{i j} C_{j}(\mu)
$$

\footnotetext{
1 This implies that the corresponding counterterms can be obtained with the same shift, which is expected because QCD corrections only affect the quark current.
} 


\section{A. Flavor-conserving operators}

We consider bilinear quark operators that involve the third generation quarks $t$ and $b$. First of all, the following operators are relevant in our calculation:

$$
\begin{aligned}
& O_{t G}=y_{t} g_{s}\left(\bar{Q} \sigma^{\mu \nu} T^{A} t\right) \tilde{\varphi} G_{\mu \nu}^{A} \\
& O_{t W}=y_{t} g_{W}\left(\bar{Q} \sigma^{\mu \nu} \tau^{I} t\right) \tilde{\varphi} W_{\mu \nu}^{I} \\
& O_{t B}=y_{t} g_{Y}\left(\bar{Q} \sigma^{\mu \nu} t\right) \tilde{\varphi} B_{\mu \nu} \\
& O_{t \varphi}=-y_{t}^{3}\left(\varphi^{\dagger} \varphi\right)(\bar{Q} t) \tilde{\varphi} .
\end{aligned}
$$

Among these operators $O_{t W}$ and $O_{t G}$ contribute to $t \rightarrow$ $b W$ decay. $O_{t \varphi}$ is needed to absorb the UV divergence in the top mass correction from $O_{t G}$. We include $O_{t B}$ only for completeness. The anomalous dimension matrix, for $C_{t G}, C_{t W}, C_{t B}$ and $C_{t \varphi}$, is

$$
\gamma=\frac{2 \alpha_{s}}{\pi}\left(\begin{array}{cccc}
\frac{1}{6} & 0 & 0 & 0 \\
\frac{1}{3} & \frac{1}{3} & 0 & 0 \\
\frac{5}{9} & 0 & \frac{1}{3} & 0 \\
-4 & 0 & 0 & -1
\end{array}\right)
$$

We can see that the mixing effects are from operator $O_{t G}$, which renormalizes all the other operators. Note that the above anomalous dimension matrix is not "closed", in the sense that $O_{t G}$ will be renormalized by for example $O_{G}=g_{s} f^{A B C} G_{\mu}^{A \nu} G_{\nu}^{B \rho} G_{\rho}^{C \mu}$ at order $\alpha_{s}[73]$.

The following four operators are similar to $O_{t G}, O_{t W}$, $O_{t B}$ and $O_{t \varphi}$, and can be obtained by $t \rightarrow b$ and $\tilde{\varphi} \rightarrow \varphi$.

$$
\begin{aligned}
& O_{b G}=y_{t} g_{s}\left(\bar{Q} \sigma^{\mu \nu} T^{A} b\right) \varphi G_{\mu \nu}^{A} \\
& O_{b W}=y_{t} g_{W}\left(\bar{Q} \sigma^{\mu \nu} \tau^{I} b\right) \varphi W_{\mu \nu}^{I} \\
& O_{b B}=y_{t} g_{Y}\left(\bar{Q} \sigma^{\mu \nu} b\right) \varphi B_{\mu \nu} \\
& O_{b \varphi}=-y_{t}^{3}\left(\varphi^{\dagger} \varphi\right)(\bar{Q} b) \varphi
\end{aligned}
$$

The operator $O_{b W}$ contributes to $t \rightarrow b W$ decay, but only at order $\Lambda^{-4}$ if the bottom-quark mass is ignored. Their anomalous dimension matrix is

$$
\gamma=\frac{2 \alpha_{s}}{\pi}\left(\begin{array}{cccc}
\frac{1}{6} & 0 & 0 & 0 \\
\frac{1}{3} & \frac{1}{3} & 0 & 0 \\
-\frac{1}{9} & 0 & \frac{1}{3} & 0 \\
0 & 0 & 0 & -1
\end{array}\right)
$$

Comparing with Eq. (69), there are two different components, $\gamma_{31}$ and $\gamma_{41}$. The difference in $\gamma_{31}$ is due to the different hypercharges of the top quark and the bottom quark, as this term is proportional to $Y_{t}+Y_{Q}$ for $O_{t W}$, but to $Y_{b}+Y_{Q}$ for $O_{b W}$, where $Y_{t}=2 / 3, Y_{b}=-1 / 3$ are the hypercharges of the right handed top quark and bottom quark, while $Y_{Q}=1 / 6$ is the hypercharge of the left-handed quark doublet. The difference in $\gamma_{41}$ is simply because we are normalizing these operators with $y_{t}$ instead of $y_{b}$ (which we neglect), and so this component is suppressed by $\left(y_{b} / y_{t}\right)^{2}$.
Finally, the following operators do not have anomalous dimension:

$$
\begin{aligned}
O_{\varphi Q}^{(3)} & =i \frac{1}{2} y_{t}^{2}\left(\varphi^{\dagger} \overleftrightarrow{D}_{\mu}^{I} \varphi\right)\left(\bar{Q} \gamma^{\mu} \tau^{I} Q\right) \\
O_{\varphi Q}^{(1)} & =i \frac{1}{2} y_{t}^{2}\left(\varphi^{\dagger} \overleftrightarrow{D}_{\mu} \varphi\right)\left(\bar{Q} \gamma^{\mu} Q\right) \\
O_{\varphi t} & =i \frac{1}{2} y_{t}^{2}\left(\varphi^{\dagger} \overleftrightarrow{D}_{\mu} \varphi\right)\left(\bar{t} \gamma^{\mu} t\right) \\
O_{\varphi b} & =i \frac{1}{2} y_{t}^{2}\left(\varphi^{\dagger} \overleftrightarrow{D}_{\mu} \varphi\right)\left(\bar{b} \gamma^{\mu} b\right) \\
O_{\varphi \varphi} & =i y_{t}^{2}\left(\tilde{\varphi}^{+} D_{\mu} \varphi\right)\left(\bar{t} \gamma^{\mu} b\right)
\end{aligned}
$$

due to current conservation. Here $O_{\varphi Q}^{(3)}$ and $O_{\varphi \varphi}$ contribute to top-quark decay.

To illustrate the mixing effects, we plot the RG evolution of $C_{t W}$ and $C_{t G}$ in Figure 2. These two operators affect the $W$-helicity fractions in $t \rightarrow b W$ decay. Their evolution at order $\mathcal{O}\left(\alpha_{s}\right)$ is determined by the anomalous dimension presented in Eq. (68), and is given here by solving the RG equations:

$$
\begin{aligned}
C_{t W}(\mu)= & C_{t W}\left(m_{t}\right)\left(\frac{\alpha_{s}(\mu)}{\alpha_{s}\left(m_{t}\right)}\right)^{-\frac{4}{3 \beta_{0}}} \\
& -2 C_{t G}\left(m_{t}\right)\left[\left(\frac{\alpha_{s}(\mu)}{\alpha_{s}\left(m_{t}\right)}\right)^{-\frac{2}{3 \beta_{0}}}-\left(\frac{\alpha_{s}(\mu)}{\alpha_{s}\left(m_{t}\right)}\right)^{-\frac{4}{3 \beta_{0}}}\right] \\
C_{t G}(\mu)= & C_{t G}\left(m_{t}\right)\left(\frac{\alpha_{s}(\mu)}{\alpha_{s}\left(m_{t}\right)}\right)^{-\frac{2}{3 \beta_{0}}}
\end{aligned}
$$

where $\beta_{0}=11-2 N_{f} / 3$. The second term on the r.h.s of the first equation implies that there is a mixing effect between these two operators. The left plot in Figure 2 shows the direction of the $\mathrm{RG}$ flows in the $C_{t W}-C_{t G}$ plane, as well as the distance between $\left(C_{t W}(\mu), C_{t G}(\mu)\right)$ at $\mu=m_{t}$ and at $\mu=2 \mathrm{TeV}$.

\section{B. Flavor Changing operators}

We consider bilinear quark operators that involve both first and third generation quarks. An interesting feature is that the $\mathcal{O}\left(\alpha_{s}\right)$ mixing will not change the flavor superscripts, due to the chirality of the massless light quarks. More specifically, operators with a (13) flavor indices will not mix with those with $(31)$, and operators with $(1+3)$ are current operators and do not have any anomalous dimension. For this reason the anomalous dimension matrix can be divided into blocks that are closed under RG evolution at $\mathcal{O}\left(\alpha_{s}\right)$, according to the type and chirality of the light quark involved in the bilinear quark operator.

1. Operators with left-handed massless up quark The following four operators mix with $O_{u G}^{(13)}$, and 

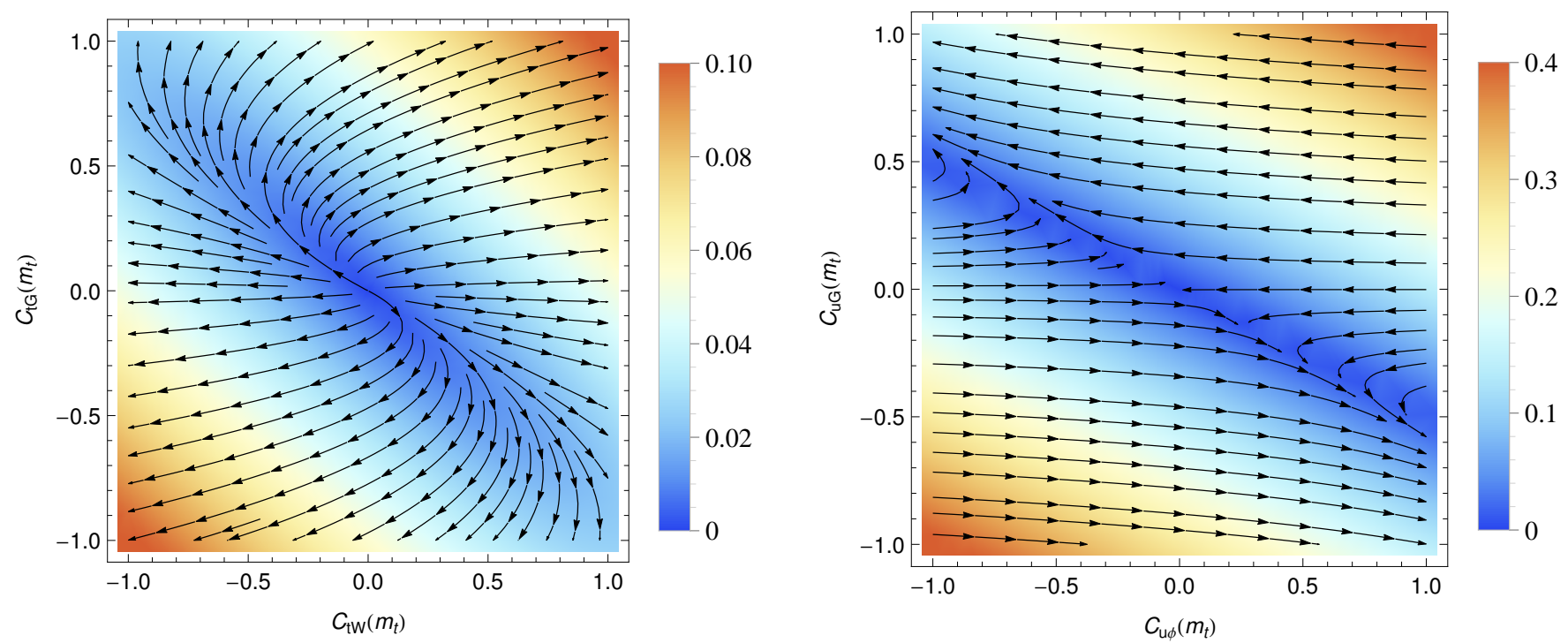

FIG. 2: Left: RG evolution of $C_{t W}$ and $C_{t G}$. The arrows represent the direction of the flow when $\mu$ increases. The color shows the distance between $\left(C_{t W}\left(m_{t}\right), C_{t G}\left(m_{t}\right)\right)$ and $\left(C_{t W}(\mu), C_{t G}(\mu)\right)$ for $\mu=2 \mathrm{TeV}$, i.e. $\left[\left(C_{t W}\left(m_{t}\right)-C_{t W}(\mu)\right)^{2}+\left(C_{t G}\left(m_{t}\right)-C_{t G}(\mu)\right)^{2}\right]^{1 / 2}$. Right: same plot but for $C_{u \varphi}$ and $C_{u G}$.

contribute to either $t \rightarrow u V$ or $t \rightarrow u h$, or both:

$$
\begin{aligned}
& O_{u G}^{(13)}=y_{t} g_{s}\left(\bar{q} \sigma^{\mu \nu} T^{A} t\right) \tilde{\varphi} G_{\mu \nu}^{A} \\
& O_{u W}^{(13)}=y_{t} g_{W}\left(\bar{q} \sigma^{\mu \nu} \tau^{I} t\right) \tilde{\varphi} W_{\mu \nu}^{I} \\
& O_{u B}^{(13)}=y_{t} g_{Y}\left(\bar{q} \sigma^{\mu \nu} t\right) \tilde{\varphi} B_{\mu \nu} \\
& O_{u \varphi}^{(13)}=-y_{t}^{3}\left(\varphi^{\dagger} \varphi\right)(\bar{q} t) \tilde{\varphi} .
\end{aligned}
$$

Their mixing is

$$
\gamma=\frac{2 \alpha_{s}}{\pi}\left(\begin{array}{cccc}
\frac{1}{6} & 0 & 0 & 0 \\
\frac{1}{3} & \frac{1}{3} & 0 & 0 \\
\frac{5}{9} & 0 & \frac{1}{3} & 0 \\
-2 & 0 & 0 & -1
\end{array}\right) .
$$

Comparing with Eq. (69), only the component $\gamma_{41}$ is different. This is because $O_{t G}$ is flavorconserving, and its contribution to the anomalous dimension comes from two diagrams, each proportional to the Yukawa coupling $y_{t}$, while for $O_{u G}^{(13)}$ one of the two diagrams would be proportional to $y_{u}$, which we neglect.

2. Operators with left-handed massless down quark

The following four operators mix with $O_{d G}^{(13)}$. They are irrelevant for top decay, but we list them for completeness:

$$
\begin{aligned}
& O_{d G}^{(13)}=y_{t} g_{s}\left(\bar{q} \sigma^{\mu \nu} T^{A} b\right) \varphi G_{\mu \nu}^{A} \\
& O_{d W}^{(13)}=y_{t} g_{W}\left(\bar{q} \sigma^{\mu \nu} \tau^{I} b\right) \varphi W_{\mu \nu}^{I} \\
& O_{d B}^{(13)}=y_{t} g_{Y}\left(\bar{q} \sigma^{\mu \nu} b\right) \varphi B_{\mu \nu} \\
& O_{d \varphi}^{(13)}=-y_{t}^{3}\left(\varphi^{\dagger} \varphi\right)(\bar{q} b) \varphi
\end{aligned}
$$

Their mixing is is given by Eq. (74).

3. Operators with right-handed massless up quark

The following four operators mix with $O_{u G}^{(31)}$, and contribute to either $t \rightarrow u V$ or $t \rightarrow u h$, or both:

$$
\begin{aligned}
& O_{u G}^{(31)}=y_{t} g_{s}\left(\bar{Q} \sigma^{\mu \nu} T^{A} u\right) \tilde{\varphi} G_{\mu \nu}^{A} \\
& O_{u W}^{(31)}=y_{t} g_{W}\left(\bar{Q} \sigma^{\mu \nu} \tau^{I} u\right) \tilde{\varphi} W_{\mu \nu}^{I} \\
& O_{u B}^{(31)}=y_{t} g_{Y}\left(\bar{Q} \sigma^{\mu \nu} u\right) \tilde{\varphi} B_{\mu \nu} \\
& O_{u \varphi}^{(31)}=-y_{t}^{3}\left(\varphi^{\dagger} \varphi\right)(\bar{Q} u) \tilde{\varphi} .
\end{aligned}
$$

Their mixing is is given by Eq. (86).

4. Operators with right-handed massless down quark 
The following four operators mix with $O_{d G}^{(31)}$ :

$$
\begin{aligned}
& O_{d G}^{(31)}=y_{t} g_{s}\left(\bar{Q} \sigma^{\mu \nu} T^{A} d\right) \varphi G_{\mu \nu}^{A} \\
& O_{d W}^{(31)}=y_{t} g_{W}\left(\bar{Q} \sigma^{\mu \nu} \tau^{I} d\right) \varphi W_{\mu \nu}^{I} \\
& O_{d B}^{(31)}=y_{t} g_{Y}\left(\bar{Q} \sigma^{\mu \nu} d\right) \varphi B_{\mu \nu} \\
& O_{d \varphi}^{(31)}=-y_{t}^{3}\left(\varphi^{\dagger} \varphi\right)(\bar{Q} d) \varphi .
\end{aligned}
$$

Note the first two contribute to flavor-changing charged-current top decay: $t \rightarrow d W$. Though we didn't study this case explicitly, it is essentially the same as $t \rightarrow b W$ and the results can be inferred. Their mixing is given by Eq. (74).

Finally, the following operators do not have anomalous dimension:

$$
\begin{aligned}
& O_{\varphi q}^{(3,1+3)}=i \frac{1}{2} y_{t}^{2}\left(\varphi^{\dagger} \overleftrightarrow{D}_{\mu}^{I} \varphi\right)\left(\bar{q} \gamma^{\mu} \tau^{I} Q\right) \\
& O_{\varphi q}^{(1,1+3)}=i \frac{1}{2} y_{t}^{2}\left(\varphi^{\dagger} \overleftrightarrow{D}_{\mu} \varphi\right)\left(\bar{q} \gamma^{\mu} Q\right) \\
& O_{\varphi u}^{(1+3)}=i \frac{1}{2} y_{t}^{2}\left(\varphi^{\dagger} \overleftrightarrow{D}_{\mu} \varphi\right)\left(\bar{t} \gamma^{\mu} u\right) \\
& O_{\varphi d}^{(1+3)}=i \frac{1}{2} y_{t}^{2}\left(\varphi^{\dagger} \overleftrightarrow{D}_{\mu} \varphi\right)\left(\bar{b} \gamma^{\mu} d\right) \\
& O_{\varphi \varphi}^{(13)}=i y_{t}^{2}\left(\tilde{\varphi}^{+} D_{\mu} \varphi\right)\left(\bar{t} \gamma^{\mu} d\right) \\
& O_{\varphi \varphi}^{(31)}=i y_{t}^{2}\left(\varphi^{+} D_{\mu} \tilde{\varphi}\right)\left(\bar{b} \gamma^{\mu} u\right) .
\end{aligned}
$$

The first three contribute to $t \rightarrow u Z$.

For illustration we plot the RG evolution of the flavorchanging Yukawa and color-dipole operators, $O_{u \varphi}$ and $O_{u G}$ (the anomalous dimension is the same for flavor structure (13) and (31) so we omit the superscript) in Figure 2. These two operators will contribute to the decay mode $t \rightarrow u_{i} h$. Their mixing effect is much larger than that of $O_{t W}$ and $O_{t G}$.

\section{Four-fermion operators with two quarks and two leptons}

Now we consider operators that contribute to semileptonic top quark decays. These operators do not mix at order $\alpha_{s}$. Their mixing with bilinear quark operators is not an order $\alpha_{s}$ effect so we neglect. In the following we give the anomalous dimensions. The flavor indices do not matter, so we will omit them. (For example, $O_{l Q}^{(3)}$ and $O_{l q}^{(3,1+3)}$ will have the same anomalous dimension at $\mathcal{O}\left(\alpha_{s}\right)$, and so here we simply write them as $O_{l q}^{(3)}$, etc.)

The V-V operators are not renormalized because of current conservation. These include

$$
\begin{aligned}
O_{l q}^{(1)} & =\left(\bar{l} \gamma_{\mu} l\right)\left(\bar{q} \gamma^{\mu} q\right) \\
O_{l q}^{(3)} & =\left(\bar{l} \gamma_{\mu} \gamma^{I} l\right)\left(\bar{q} \gamma^{\mu} \tau^{I} q\right) \\
O_{e u} & =\left(\bar{e} \gamma_{\mu} e\right)\left(\bar{u} \gamma^{\mu} u\right) \\
O_{e d} & =\left(\bar{e} \gamma_{\mu} e\right)\left(\bar{d} \gamma^{\mu} d\right) \\
O_{l u} & =\left(\bar{l} \gamma_{\mu} l\right)\left(\bar{u} \gamma^{\mu} u\right) \\
O_{l d} & =\left(\bar{l} \gamma_{\mu} l\right)\left(\bar{d} \gamma^{\mu} d\right) \\
O_{q e} & =\left(\bar{q} \gamma_{\mu} q\right)\left(\bar{e} \gamma^{\mu} e\right) .
\end{aligned}
$$

The S-S operators with a scalar quark current have anomalous dimension

$$
\gamma=-2 \frac{\alpha_{s}}{\pi}
$$

These include

$$
\begin{aligned}
& O_{\text {ledq }}=(\bar{l} e)(\bar{d} q) \\
& O_{\text {lequ }}^{(1)}=(\bar{l} e) \varepsilon(\bar{q} u) .
\end{aligned}
$$

Finally, the T-T operators with a tensor quark current have anomalous dimension

$$
\gamma=\frac{2 \alpha_{s}}{3 \pi} .
$$

There is only one such operator:

$$
O_{l e q u}^{(3)}=\left(\bar{l} \sigma_{\mu \nu} e\right) \varepsilon\left(\bar{q} \sigma^{\mu \nu} u\right) .
$$

\section{ANALYTICAL RESULTS}

\section{A. Decay mode: $t \rightarrow b W$}

In this decay mode, the fractions of $W$ bosons produced with certain helicities are sensitive to the structure of $t b W$ vertex. Measurements on helicity fractions can provide information about new physics. In Ref. [56], the QCD corrections to the $W$ boson helicity fractions with a general anomalous $t b W$ vertex are calculated. Our results on the partial decay width are in agreement with Ref. [56]. Here we will present these results, and also include one additional operator, $O_{t G}$. This operator has no tree level contribution and has been ignored in previous calculations. However, it gives rise to a chromo-magnetic moment of the top quark, and thus modifies the standard QCD correction to the SM $t \rightarrow b W$ decay process. It also has a mixing with the other operators, and so needs to be included.

In the following we present the full NLO results for topquark decay to bottom and $W$ boson in a certain helicity state. The Feynman diagrams are given in Figure 3. We give expressions for $\Gamma^{(t o t)}, \Gamma^{(L)}, \Gamma^{(T)}$ and $\Gamma^{(F)}$, where the superscripts represent the total width, longitudinal partial width, transverse partial width, and the difference 


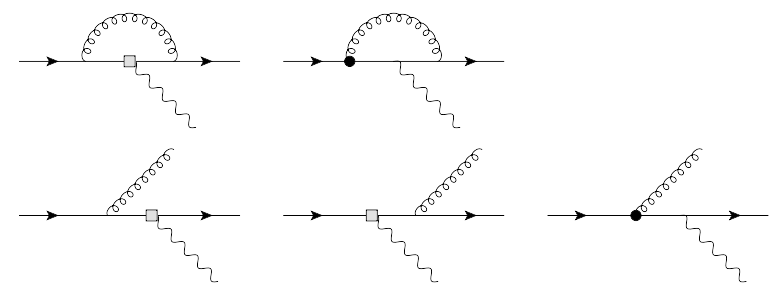

FIG. 3: Virtual and real corrections for $t \rightarrow b W$. The black dots represent interactions arising from color-dipole operator $O_{t G}$, while squares represent interactions from all the other operators, which modify the $t b W$ vertex. There will be additional diagrams if one includes a color-dipole operator for the bottom quark. These diagrams will not interfere with the SM contribution in the limit of $m_{b}=0$. between transverse positive and transverse negative partial widths. The decay width of each helicity state (which we denote by + , - and 0 ) is thus given by

$$
\begin{aligned}
& \Gamma^{(+)}=\frac{\Gamma^{(T)}+\Gamma^{(F)}}{2} \\
& \Gamma^{(0)}=\Gamma^{(L)} \\
& \Gamma^{(-)}=\frac{\Gamma^{(T)}-\Gamma^{(F)}}{2} .
\end{aligned}
$$

For later convenience, we write the full results as functions of the $W$-boson mass and the coefficients of dimension-six operators:

$$
\begin{aligned}
\Gamma_{b W}^{(t o t, L, T)} & \equiv \Gamma_{b W}^{(t o t, L, T)}\left(x, C_{\varphi Q}^{(3)}, C_{\varphi \varphi}, C_{t W}, C_{b W}, C_{t G}\right) \\
& =\Gamma_{\mathrm{SM}}^{(t o t, L, T)}(x)+\Gamma_{1}^{(t o t, L, T)}\left(x, C_{\varphi Q}^{(3)}, C_{t W}, C_{t G}\right)+\Gamma_{2}^{(t o t, L, T)}\left(x, C_{\varphi Q}^{(3)}, C_{t W}\right)+\Gamma_{2}^{(t o t, L, T)}\left(x, C_{\varphi \varphi} / 2, C_{b W}\right) \\
\Gamma_{b W}^{(F)} & \equiv \Gamma_{b W}^{(F)}\left(x, C_{\varphi Q}^{(3)}, C_{\varphi \varphi}, C_{t W}, C_{b W}, C_{t G}\right) \\
& =\Gamma_{\mathrm{SM}}^{(F)}(x)+\Gamma_{1}^{(F)}\left(x, C_{\varphi Q}^{(3)}, C_{t W}, C_{t G}\right)+\Gamma_{2}^{(F)}\left(x, C_{\varphi Q}^{(3)}, C_{t W}\right)-\Gamma_{2}^{(F)}\left(x, C_{\varphi \varphi} / 2, C_{b W}\right)
\end{aligned}
$$

where $x=m_{W} / m_{t}, \Gamma_{\mathrm{SM}}$ represents the SM contribution, $\Gamma_{1}$ is the contribution from the interference of SM and dimension-six operators, and $\Gamma_{2}$ is the squared contribution from dimension-six operators. We include the coefficient $C_{t G}$ only in the interference term $\Gamma_{1}$. A complete calculation of $\mathcal{O}\left(C_{t G}^{2}\right)$ effects requires counterterms from dimensioneight operators (for example, the diagram in Figure 1 but with two dots representing $O_{t G}$ may need to be regulated by a dimension-eight counterterm), and so we will not consider in this work. The functions $\Gamma_{\mathrm{SM}, 1,2}$ are given by

$$
\begin{gathered}
\Gamma_{\mathrm{SM}}^{(i)}(x)=\frac{\alpha m_{t}}{4 s^{2}} \Gamma_{V, 0}^{(i)}(x)\left(1+\alpha_{s} \delta_{V}^{i}(x)\right) \\
\Gamma_{1}^{(i)}\left(x, c_{V}, c_{T}, c_{G}\right)=\frac{\alpha m_{t}^{3}}{s^{2} \Lambda^{2}}\left[\operatorname{Re}\left(c_{V}\right) \Gamma_{V, 0}^{(i)}(x)\left(1+\alpha_{s} \delta_{V}^{i}(x)\right)+\operatorname{Re}\left(c_{T}\right) \Gamma_{V T, 0}^{(i)}(x)\left(1+\alpha_{s} \delta_{V T}^{i}(x)\right)\right. \\
\left.+\operatorname{Re}\left(c_{G}\right) \Gamma_{V T, 0}(x)^{(i)}\left(\alpha_{s} \delta_{V G}^{i}(x)\right)\right] \\
\Gamma_{2}^{(i)}\left(x, c_{V}, c_{T}\right)=\frac{\alpha m_{t}^{5}}{s^{2} \Lambda^{4}}\left[\left|c_{V}\right|^{2} \Gamma_{V, 0}^{(i)}(x)\left(1+\alpha_{s} \delta_{V}^{i}(x)\right)+\left|c_{T}\right|^{2} \Gamma_{T, 0}^{(i)}(x)\left(1+\alpha_{s} \delta_{T}^{i}(x)\right)\right. \\
\left.+2 \operatorname{Re}\left(c_{V} c_{T}^{*}\right) \Gamma_{V T, 0}(x)^{(i)}\left(1+\alpha_{s} \delta_{V T}^{i}(x)\right)\right]
\end{gathered}
$$

where $s=\sin \theta_{W}$ is the sine of the weak angle $\theta_{W}$, and the functions $\Gamma_{j, 0}^{(i)}(x)$ represent tree level contributions:

$$
\begin{aligned}
\Gamma_{V, 0}^{(t o t)}(x) & =\frac{\left(x^{2}-1\right)^{2}\left(2 x^{2}+1\right)}{4 x^{2}} \\
\Gamma_{V T, 0}^{(t o t)}(x) & =\frac{3}{2}\left(x^{2}-1\right)^{2} \\
\Gamma_{T, 0}^{t o t}(x) & =\left(x^{2}-1\right)^{2}\left(x^{2}+2\right)
\end{aligned}
$$




$$
\begin{gathered}
\Gamma_{V, 0}^{(T)}(x)=\frac{1}{2}\left(x^{2}-1\right)^{2} \\
\Gamma_{V T, 0}^{(T)}(x)=\left(x^{2}-1\right)^{2} \\
\Gamma_{T, 0}^{(T)}(x)=2\left(x^{2}-1\right)^{2} \\
\Gamma_{V, 0}^{(F)}(x)=-\frac{1}{2}\left(x^{2}-1\right)^{2} \\
\Gamma_{V T, 0}^{(F)}(x)=-\left(x^{2}-1\right)^{2} \\
\Gamma_{T, 0}^{(F)}(x)=-2\left(x^{2}-1\right)^{2},
\end{gathered}
$$

and the functions $\delta_{V, T, V T, V G}^{i}(x)$ represent $\mathrm{O}\left(\alpha_{s}\right)$ corrections. Their expressions are given in Appendix A. Note that these results apply even if the $W$-boson is off shell, with $x=m_{W^{*}} / m_{t}$.

\section{B. Decay mode $t \rightarrow u_{i} V$}

In this section we consider the flavor-changing decay mode $t \rightarrow u_{i} V$ mediated by dimension-six operators. In the SM the flavor-changing neutral couplings involving the top quark are loop-induced and have a strong Glashow-Iliopoulos-Maiani mechanism suppression, leading to negligible branching ratios [74-76]. Therefore the observation of such processes will provide a clear signal of new physics.

The QCD corrections have been studied in the literature. In Ref. [59], the contributions of dipole couplings to $t \rightarrow u_{i}+\gamma / Z$ and $t \rightarrow u_{i}+g$ have been investigated at NLO in QCD. The vector-type couplings for the $t u Z$ vertex are considered in Refs. [57, 58]. Furthermore, Refs. $[57,58]$ also presented the anomalous dimensions, in terms of dimension-four and dimension-five effective Lagrangian, (hence without explicit $S U(2)_{L} \times U(1)_{Y}$ symmetry). For $t \rightarrow u_{i}+Z$, only the total width, i.e. with all polarization states summed over, is available in these works. The Feynman diagrams for these processes are shown in Figure 4 and 5.

Here we give the partial decay width of $t \rightarrow u Z^{*}$ for each helicity state of an off-shell $Z$ boson, in terms of dimension-six operators. The helicity decay rates are needed to derive the differential rates of the three-body decays. Our total widths are in agreement with previous results in Refs. [57, 58], only if we change the sign of $\hat{a}$ for the contributions from the $O_{u G}^{(13)}$ operator. Again, for later convenience we write the full results as functions of the $Z$-boson mass and the coefficients of dimension-six operators:

$\Gamma_{u Z}^{(t o t, L, T, F)} \equiv \Gamma_{u Z}^{(t o t, L, T, F)}\left(x, \hat{v}, \hat{a} ; C_{\varphi q}^{(1,1+3)}-C_{\varphi q}^{(3,1+3)},-s^{2} C_{u B}^{(13)}+c^{2} C_{u W}^{(13)}, C_{u G}^{(13)} ; C_{\varphi u}^{(1+3)},-s^{2} C_{u B}^{(31) *}+c^{2} C_{u W}^{(31) *}, C_{u G}^{(31) *}\right)$

where $\hat{v}=1 / 2-4 s^{2} / 3, \hat{a}=1 / 2$ are the vector and axial-vector coupling constants of $Z u \bar{u}, c=\cos \theta_{W}$, and the function $\Gamma_{u Z}^{(i)}$ is defined as

$$
\begin{aligned}
\Gamma_{u Z}^{(t o t, L, T)}\left(x, v, a ; c_{V}^{L}, c_{T}^{L}, c_{G}^{L} ; c_{V}^{R}, c_{T}^{R}, c_{G}^{R}\right) & =\Gamma_{V}^{(t o t, L, T)}\left(x, v, a ; c_{V}^{L}, c_{T}^{L}, c_{G}^{L}\right)+\Gamma_{V}^{(t o t, L, T)}\left(x, v,-a ; c_{V}^{R}, c_{T}^{R}, c_{G}^{R}\right) \\
\Gamma_{u Z}^{(F)}\left(x, v, a ; c_{V}^{L}, c_{T}^{L}, c_{G}^{L} ; c_{V}^{R}, c_{T}^{R}, c_{G}^{R}\right) & =\Gamma_{V}^{(F)}\left(x, v, a ; c_{V}^{L}, c_{T}^{L}, c_{G}^{L}\right)-\Gamma_{V}^{(F)}\left(x, v,-a ; c_{V}^{R}, c_{T}^{R}, c_{G}^{R}\right),
\end{aligned}
$$

where the functions $\Gamma_{V}^{(i)}$ are given by

$$
\begin{aligned}
& \Gamma_{V}^{(i)}\left(x, v, a ; c_{V}, c_{T}, c_{G}\right) \\
= & \frac{\alpha m_{t}^{5}}{2 s^{2} c^{2} \Lambda^{4}}\left\{\frac{1}{4}\left|c_{V}\right|^{2} \Gamma_{V, 0}^{(i)}(x)\left[1+\alpha_{s} \delta_{V}^{i}(x)\right]+\left|c_{T}\right|^{2} \Gamma_{T, 0}^{(i)}(x)\left[1+\alpha_{s} \delta_{T}^{i}(x)\right]-\operatorname{Re}\left(c_{V} c_{T}^{*}\right) \Gamma_{V T, 0}^{(i)}\left[1+\alpha_{s} \delta_{V T}^{i}(x)\right]\right. \\
& -\operatorname{Re}\left(c_{V} c_{G}^{*}\right) \Gamma_{V T, 0}^{(i)} \alpha_{s}\left[\delta_{V G, r}^{i}(x, \operatorname{Re}(v), a)-\delta_{V G, i}^{i}(x, \operatorname{Im}(v), 0)\right]-\operatorname{Im}\left(c_{V} c_{G}^{*}\right) \Gamma_{V T, 0}^{(i)} \alpha_{s}\left[\delta_{V G, i}^{i}(x, \operatorname{Re}(v), a)+\delta_{V G, r}^{i}(x, \operatorname{Im}(v), 0)\right] \\
& +2 \operatorname{Re}\left(c_{T} c_{G}^{*}\right) \Gamma_{T, 0}^{(i)} \alpha_{s}\left[\delta_{T G, r}^{i}(x, \operatorname{Re}(v), a)-\delta_{T G, i}^{i}(x, \operatorname{Im}(v), 0)\right]+2 \operatorname{Im}\left(c_{T} c_{G}^{*}\right) \Gamma_{T, 0}^{(i)} \alpha_{s}\left[\delta_{T G, i}^{i}(x, \operatorname{Re}(v), a)+\delta_{T G, r}^{i}(x, \operatorname{Im}(v), 0)\right] \\
& \left.+\left|c_{G}\right|^{2} \Gamma_{T, 0}^{(i)}(x) \alpha_{s}\left[\delta_{G 2}^{i}(x, \operatorname{Re}(v), a)+\delta_{G 2}^{i}(x, \operatorname{Im}(v), 0)\right]\right\}
\end{aligned}
$$

where the tree level contributions, $\Gamma_{j, 0}^{(i)}$ are given in Eq. (125)-(136), and the NLO corrections, $\delta^{i}(x)$ 's, are given in Appendix A. Note that in the above formula we allow for a complex value of $\hat{v}$, even though $\hat{v}$ is real in the SM. This will be useful when we consider four-fermion operators in the next section.

We have also checked the available results for $t \rightarrow u_{i} g$ in Ref. [59] and for $t \rightarrow u_{i} \gamma$ in Ref. [57, 58], and find 


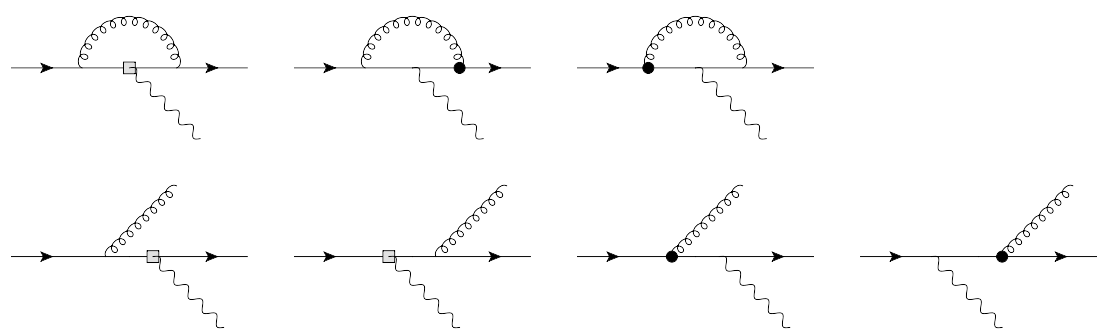

FIG. 4: Feynman diagrams for $t \rightarrow u+\gamma / Z$. The black dots represent interactions arising from color-dipole operators $O_{u G}^{(13,31)}$, while squares represent interactions from all the other operators, which modify the $t u V$ vertex.
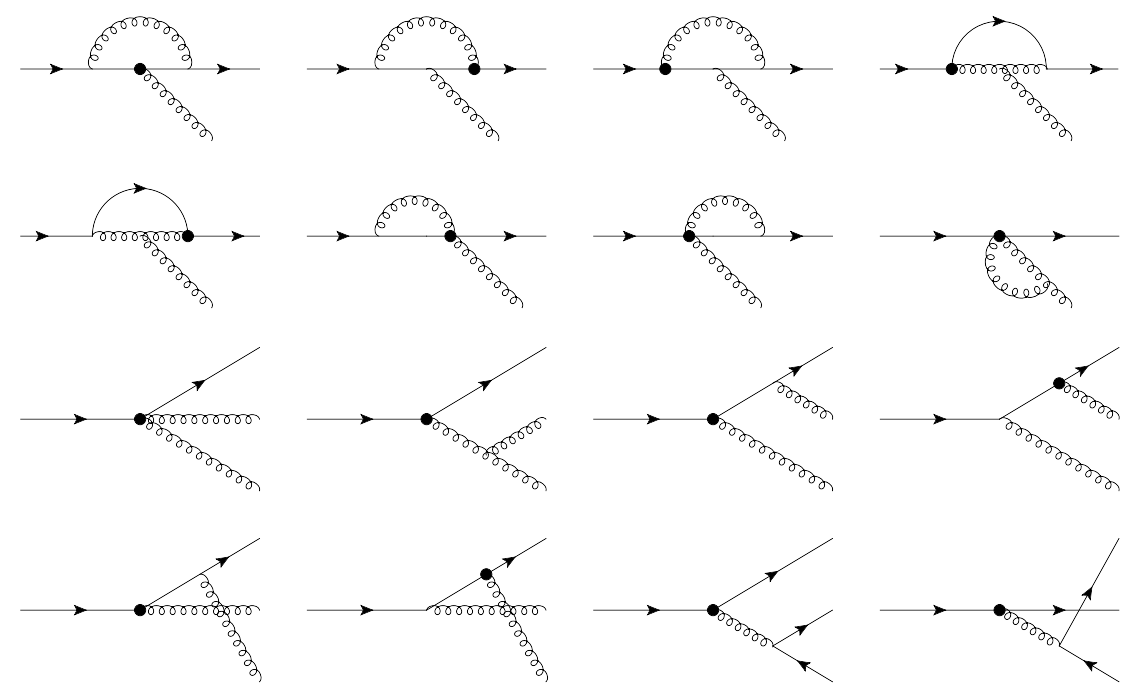

FIG. 5: Feynman diagrams for $t \rightarrow u+g$. The black dots represent interactions arising from color-dipole operators $O_{u G}^{(13,31)}$.

agreement. For the sake of completeness, we present these results here using our formalism.

For $t \rightarrow u_{i} g$ :

$$
\Gamma_{u g}=\frac{4 \alpha_{s} m_{t}^{5}}{3 \Lambda^{4}}\left(\left|C_{u G}^{(13)}\right|^{2}+\left|C_{u G}^{(31)}\right|^{2}\right)\left(1+\alpha_{s} \delta_{G}^{g}\right)
$$

with

$\delta_{G}^{g}=-\frac{1}{72 \pi}\left[6\left(29-2 N_{f}\right) \log \frac{m_{t}^{2}}{\mu^{2}}+36 N_{f}-749+38 \pi^{2}\right]$,

where $N_{f}=5$ is the number of running flavors in $g_{s}$. For $t \rightarrow u_{i} \gamma$ :

$$
\begin{aligned}
\Gamma_{u \gamma} & =\Gamma_{\gamma}\left(\hat{x}, \hat{y} ; C_{u W}^{(13)}+C_{u B}^{(13)}, C_{u G}^{(13)}\right) \\
& +\Gamma_{\gamma}\left(\hat{x}, \hat{y} ; C_{u W}^{(31) *}+C_{u B}^{(31) *}, C_{u G}^{(31) *}\right),
\end{aligned}
$$

where $\hat{x}$ and $\hat{y}$ are kinematic cuts on the photon energy and the jet-photon separation, required to remove the photon soft-collinear divergences:

$$
\begin{array}{r}
1-\mathbf{p}_{\gamma} \cdot \mathbf{p}_{u_{i}} / E_{\gamma} E_{u_{i}}>\hat{x} \\
2 E_{\gamma} / m_{t}>\hat{y},
\end{array}
$$

where the energy $E_{\gamma}, E_{u_{i}}$ and three-momenta $\mathbf{p}_{\gamma}, \mathbf{p}_{u_{i}}$ are defined in the top-quark rest frame, see [58] for details. The function $\Gamma_{\gamma}$ is defined as 


$$
\begin{aligned}
& \Gamma_{\gamma}\left(\hat{x}, \hat{y} ; c_{T}, c_{G}\right) \\
= & \frac{\alpha m_{t}^{5}}{\Lambda^{4}}\left\{\left|c_{T}\right|^{2}\left[1+\alpha_{s} \delta_{T}^{\gamma}(\hat{x}, \hat{y})\right]+2 \operatorname{Re}\left(c_{T} c_{G}^{*}\right) \alpha_{s} \delta_{T G, r}^{\gamma}(\hat{x}, \hat{y})+2 \operatorname{Im}\left(c_{T} c_{G}^{*}\right) \alpha_{s} \delta_{T G, i}^{\gamma}(\hat{x}, \hat{y})+\left|c_{G}\right|^{2} \alpha_{s} \delta_{G}^{i}(\hat{x}, \hat{y})\right\},
\end{aligned}
$$

where the NLO corrections, $\delta_{i}^{\gamma}(\hat{x}, \hat{y})$, are given in Appendix A. Note that in both $t \rightarrow u Z$ and $t \rightarrow u \gamma$, the contributions from color-dipole operators $O_{u G}^{(13,31)}$ are pure NLO effects.

\section{Decay mode: $t \rightarrow u_{i} h$}

The QCD correction to $t \rightarrow u_{i} h$ decay through Yukawa operators was first computed in Ref. [60]. The process considered there is the charged Higgs decay of the top quark, but the QCD correction is the same (after taking into account the difference between renormalization schemes). More recently we presented a calculation for $t \rightarrow u_{i} h$ in Ref. [42], adding the contributions from the color-dipole operators $O_{u G}^{(13,31)}$, and their interferences with the Yukawa operators $O_{u \varphi}^{(13,31)}$. The Feynman diagrams are listed in Figure 6. For completeness we present here the full results for this decay mode:

$$
\begin{aligned}
\Gamma_{u h}= & \Gamma_{S}\left(x, C_{u \varphi}^{(13)}, C_{u G}^{(13)}\right) \\
& +\Gamma_{S}\left(x, C_{u \varphi}^{(31)}, C_{u G}^{(31)}\right),
\end{aligned}
$$

where the function $\Gamma_{S}$ is defined as

$\Gamma_{S}\left(x, c_{S}, c_{G}\right) \equiv\left(\frac{G_{F} m_{t}^{7}}{4 \sqrt{2} \pi \Lambda^{4}}\right)\left\{\left|c_{S}\right|^{2} \Gamma_{S, 0}(x)\left[1+\alpha_{s} \delta_{S}(x)\right]+2 \operatorname{Re}\left(c_{S} c_{G}^{*}\right) \Gamma_{S, 0}(x)\left[\alpha_{s} \delta_{S G}(x)\right]+\left|c_{G}\right|^{2} \Gamma_{S, 0}(x)\left[\alpha_{s} \delta_{G 3}(x)\right]\right\}$

where $x=m_{h} / m_{t}$, and

$$
\Gamma_{S, 0}(x) \equiv\left(1-x^{2}\right)^{2}
$$

represents the LO contribution. The $\delta_{S, S G, G 3}(x)$ functions are the NLO corrections, and are given in Appendix A. Again, here the contributions the color-dipole operators are pure NLO effects.

\section{Three-body final state}

In this section we present results for three-body final states, i.e. $t \rightarrow b e^{+} \nu$ and $t \rightarrow u l^{+} l^{-}$. The final state leptons will have the same chirality if the contributions come from two-fermion operators or $\mathrm{V}-\mathrm{V}$ operators, or opposite chiralities if they come from S-S or T-T operators. Since there is no interference between the two cases, the most convenient way of presenting our results is to consider them separately.

We first present results for same chirality leptons. We will give expressions for the differential decay rate, $\mathrm{d} \Gamma / \mathrm{d} Q^{2} \mathrm{~d} \cos \theta$, in terms of the two-body NLO results. Here $Q^{2}$ is the invariant mass of the lepton pair, and $\theta$ is the angle between the three-momentum of the antilepton in the $W$ rest frame and the $W$ momentum in top-quark rest frame. We will make use of the functions $\Gamma_{b W}$ and $\Gamma_{u V}$ defined in section IV A and IV B.

For the charged-current decay $t \rightarrow b e^{+} \nu$, the result follows immediately from section II D:

$$
\begin{aligned}
& \frac{\mathrm{d} \Gamma_{b e^{+} \nu}}{\mathrm{d} Q^{2} \mathrm{~d} \cos \theta}=\frac{Q^{2}}{16 \pi^{2}} \frac{g^{2}}{2}\left|D\left(Q^{2}, m_{W}, \Gamma_{W}\right)\right|^{-2} \times \\
& \sum_{i=+, 0,-} \Gamma_{b W}^{(i)}\left(x, C_{\varphi Q}^{(3)}{ }^{\prime}, C_{\varphi \varphi}, C_{t W}, C_{b W}, C_{t G}\right) f_{i}(\cos \theta),
\end{aligned}
$$

where

$$
x^{2}=Q^{2} / m_{t}^{2}
$$

and

$$
C_{\varphi Q}^{(3)^{\prime}}=C_{\varphi Q}^{(3)}+\frac{4}{m_{t}^{2} g^{2}} C_{l Q}^{(3)} D\left(Q^{2}, m_{W}, \Gamma_{W}\right)
$$

The above substitution takes into account the fourfermion operator $O_{l q}^{(3)}$.

Now we turn to the neutral current case. This can be derived from the two-body decay results for $t \rightarrow u Z^{*}$ and $t \rightarrow u \gamma^{*}$, where $t \rightarrow u Z^{*}$ has been given in the previous section, and $t \rightarrow u \gamma^{*}$ can be written as: 

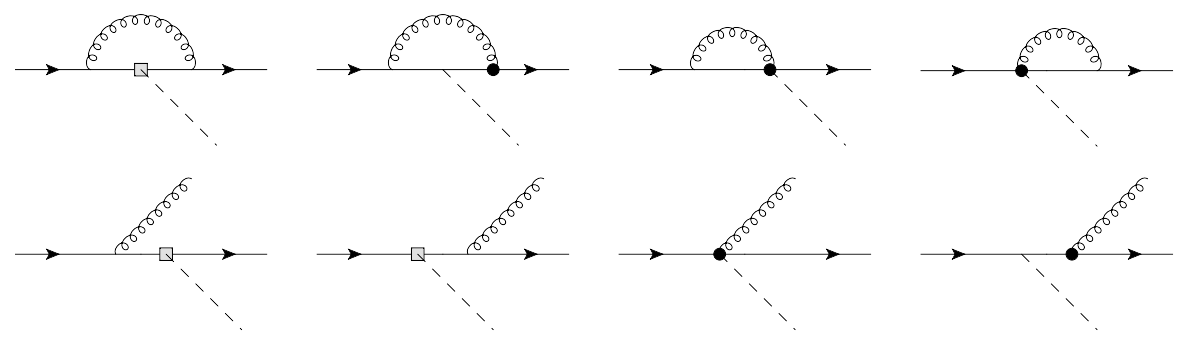

FIG. 6: Virtual and real corrections for $t \rightarrow u h$. The black dots represent interactions arising from color-dipole operators $O_{u G}^{(13,31)}$, while squares represent interactions from Yukawa operators $O_{u \varphi}^{(13,31)}$.

$$
\Gamma_{u \gamma^{*}}^{(+, 0,-)}=\Gamma_{u Z}^{(+, 0,-)}\left(Q^{2}, \frac{4}{3} s c, 0 ; 0, s c\left(C_{u B}^{(13)}+C_{u W}^{(31)}\right), C_{u G}^{(13)} ; 0, s c\left(C_{u B}^{(31) *}+C_{u W}^{(31) *}\right), C_{u G}^{(31) *}\right)
$$

However the situation is complicated by the interference between $t \rightarrow u \gamma^{*}$ and $t \rightarrow u Z^{*}$, due to their common semi-leptonic final state. The factorization of the decay amplitude can be done in a similar way as for $t \rightarrow b W^{*}$, but one needs to consider the sum of $M^{(u Z)}$ and $M^{(u \gamma)}$ :

$$
\begin{aligned}
M^{\left(u l^{+} l^{-}\right)}= & -\sum_{i=+, 0,-}\left[M_{i}^{(u Z)}\left(Q^{2}\right) \frac{g}{c}\left(T_{3}^{l}-s_{W}^{2} Q^{l}\right) D^{-1}\left(Q^{2}, m_{Z}, \Gamma_{Z}\right)+M_{i}^{(u \gamma)}\left(Q^{2}\right) e Q^{l} D^{-1}\left(Q^{2}, 0,0\right)\right] \times L_{i}^{L} \\
& -\sum_{i=+, 0,-}\left[M_{i}^{(u Z)}\left(Q^{2}\right) \frac{g}{c}\left(-s_{W}^{2} Q^{l}\right) D^{-1}\left(Q^{2}, m_{Z}, \Gamma_{Z}\right)+M_{i}^{(u \gamma)}\left(Q^{2}\right) e Q^{l} D^{-1}\left(Q^{2}, 0,0\right)\right] \times L_{i}^{R},
\end{aligned}
$$

where $T_{3}^{l}$ and $Q^{l}$ are the isospin and electric charge of the final state leptons $l^{+} l^{-}$, and $L_{i}^{L, R}$ are the polarized lepton currents. Note that there is no interference between $L_{i}^{L}$ and $L_{i}^{R}$, and the square of $L_{i}^{R}$ is

$$
\int \frac{\mathrm{d} \phi}{4 \pi}\left(L_{i}^{R} L_{j}^{R *}\right)=Q^{2} \delta_{i j} f_{i}(-\cos \theta) .
$$

For the final state with left-handed leptons, one needs the square of the combination

$$
M_{i}^{(u Z)}\left(Q^{2}\right)+M_{i}^{(u \gamma)}\left(Q^{2}\right) \frac{e Q^{l} D\left(Q^{2}, m_{Z}, \Gamma_{Z}\right)}{\frac{g}{c}\left(T_{3}^{l}-s_{W}^{2} Q^{l}\right) D\left(Q^{2}, 0,0\right)} .
$$

For the right-handed leptons the same is true if we set $T_{3}^{l}=0$. There is no need to compute the interference between $M_{i}^{(u Z)}$ and $M_{i}^{(u \gamma)}$ : we can write $M_{i}^{(u \gamma)}$ in terms of $M_{i}^{(u Z)}$, as in Eq. (153), and directly combine the couplings. This is because $M_{i}^{(u Z)}$ is a linear function of $v C_{u G}^{(13,31)}, a C_{u G}^{(13,31)}$, and other operators coefficients. In the meantime, the contributions from the four-fermion operators can be included by a shift in suitable couplings. The final result is 


$$
\begin{aligned}
\frac{\mathrm{d} \Gamma_{u l^{+} l^{-}}}{\mathrm{d} Q^{2} \mathrm{~d} \cos \theta}= & \frac{Q^{2}}{16 \pi^{2}}\left|D\left(Q^{2}, m_{Z}, \gamma_{Z}\right)\right|^{-2} \times \\
& \left\{\left[\frac{g}{c}\left(T_{3}^{l}-s_{W}^{2} Q^{l}\right)\right]^{2} \sum_{i=+, 0,-} f^{i}(\cos \theta) \times \Gamma_{u Z}^{(i)}\left(x, v^{\prime}, a ; c_{V}^{L \prime}, c_{T}^{L \prime}, c_{G}^{L} ; c_{V}^{R \prime}, c_{T}^{R \prime}, c_{G}^{R}\right)\right. \\
& \left.+\left[\frac{g}{c}\left(-s_{W}^{2} Q^{l}\right)\right]^{2} \sum_{i=+, 0,-} f^{i}(-\cos \theta) \times \Gamma_{u Z}^{(i)}\left(x, v^{\prime \prime}, a ; c_{V}^{L \prime \prime}, c_{T}^{L \prime \prime}, c_{G}^{L} ; c_{V}^{R \prime \prime}, c_{T}^{R \prime \prime}, c_{G}^{R}\right)\right\},
\end{aligned}
$$

where $x^{2}=Q^{2} / m_{t}^{2}$, and

$$
\begin{aligned}
c_{V}^{L \prime}= & C_{\varphi q}^{(1,1+3)}-C_{\varphi q}^{(3,1+3)}-\frac{2 c^{2}}{m_{t}^{2} g^{2}} \frac{D\left(Q^{2}, m_{Z}, \Gamma_{Z}\right)}{T_{3}^{l}-s_{W}^{2} Q^{l}} \\
& \times\left(C_{l q}^{(1,1+3)}+2 T_{3}^{l} C_{l q}^{(3,1+3)}\right) \\
c_{V}^{R \prime}= & C_{\varphi u}^{(1+3)}-\frac{2 c^{2}}{m_{t}^{2} g^{2}} \frac{D\left(Q^{2}, m_{Z}, \Gamma_{Z}\right)}{T_{3}^{l}-s_{W}^{2} Q^{l}} C_{l u}^{(1+3)} \\
c_{T}^{L \prime}= & -s^{2} C_{u B}^{(13)}+c^{2} C_{u W}^{(13)}+r_{L}\left(Q^{2}\right)\left(C_{u B}^{(13)}+C_{u W}^{(13)}\right) \\
c_{T}^{R \prime}= & -s^{2} C_{u B}^{(31) *}+c^{2} C_{u W}^{(31) *}+r_{L}\left(Q^{2}\right)\left(C_{u B}^{(31) *}+C_{u W}^{(31) *}\right) \\
c_{V}^{L \prime \prime}= & C_{\varphi q}^{(1,1+3)}-C_{\varphi q}^{(3,1+3)}-\frac{2 c^{2}}{m_{t}^{2} g^{2}} \frac{D\left(Q^{2}, m_{Z}, \Gamma_{Z}\right)}{-s_{W}^{2}} Q^{l} C_{q e}^{(1+3)} \\
c_{V}^{R \prime \prime}= & C_{\varphi u}^{(1+3)}-\frac{2 c^{2}}{m_{t}^{2} g^{2}} \frac{D\left(Q^{2}, m_{Z}, \Gamma_{Z}\right)}{-s_{W}^{2}} Q^{l} C_{e u}^{(1+3)} \\
c_{T}^{L \prime \prime}= & -s^{2} C_{u B}^{(13)}+c^{2} C_{u W}^{(13)}+r_{R}\left(Q^{2}\right)\left(C_{u B}^{(13)}+C_{u W}^{(13)}\right) \\
c_{T}^{R \prime \prime}= & -s^{2} C_{u B}^{(31) *}+c^{2} C_{u W}^{(31) *}+r_{R}\left(Q^{2}\right)\left(C_{u B}^{(31) *}+C_{u W}^{(31) *}\right)
\end{aligned}
$$

$$
\begin{aligned}
c_{G}^{L} & =C_{u G}^{(13)} \\
c_{G}^{R} & =C_{u G}^{(31) *} \\
v^{\prime} & =\frac{1}{2}-\frac{4}{3} s_{W}^{2}+\frac{4}{3} r_{L}\left(Q^{2}\right) \\
v^{\prime \prime} & =\frac{1}{2}-\frac{4}{3} s_{W}^{2}+\frac{4}{3} r_{R}\left(Q^{2}\right) \\
a & =\frac{1}{2} \\
r_{L}\left(Q^{2}\right) & =\frac{s^{2} c^{2} Q^{l}}{T_{3}^{l}-s^{2} Q^{l}} \frac{D\left(Q^{2}, m_{Z}, \Gamma_{Z}\right)}{D\left(Q^{2}, 0,0\right)} \\
r_{R}\left(Q^{2}\right) & =-c^{2} \frac{D\left(Q^{2}, m_{Z}, \Gamma_{Z}\right)}{D\left(Q^{2}, 0,0\right)} .
\end{aligned}
$$

This formula includes contributions from two-fermion operators with $u t \gamma, u t Z$ and $u t g$ couplings, and from fourfermions operators, as well as their interferences.

Now we move on to the case where the final state leptons have opposite chiralities.

The scalar mediated case is straightforward, as there is no angular correlation between $t \rightarrow u h^{*}$ and $h^{*} \rightarrow X$, where $X$ is some Higgs decay final state. In general, if $X$ does not involve any colored state, one can factorize the decay rate:

$$
\frac{\mathrm{d} \Gamma_{u X}}{\mathrm{~d} Q^{2}}=\frac{\sqrt{Q^{2}}}{\pi} \Gamma_{t \rightarrow u h^{*}} \Gamma_{h^{*} \rightarrow X} \mid D\left(Q^{2}, m_{h},\left.\Gamma_{h}\right|^{-2} .\right.
$$

There is no angular distribution in the $h^{*}$ rest frame. Furthermore, for semi-leptonic final states, there is no contribution from two-body decay, because the Higgs does not couple to leptons in the massless limit. Thus we only need to consider the contributions from four-fermion operators, S-S operators $O_{\text {lebQ }}, O_{\text {leQt }}^{(1)}, O_{\text {lequ }}^{(1,13)}$ and $O_{\text {lequ }}^{(1,31)}$, and T-T operators $O_{\text {leQt }}^{(3)}, C_{\text {lequ }}^{(3,13)}$ and $C_{\text {lequ }}^{(3,31)}$. Their anomalous dimensions are $-2 \alpha_{s} / \pi$ and $2 \alpha_{s} /(3 \pi)$ respectively, and there is no mixing among them. There are also contributions from the interference of S-S and T-T operators, i.e. the interferences between operators $O_{l e Q t}^{(3)}$ and $O_{\text {leQt }}^{(1)}, O_{\text {lequ }}^{(3,13)}$ and $O_{\text {lequ }}^{(1,13)}$, and $O_{\text {lequ }}^{(3,31)}$ and $O_{\text {lequ }}^{(1,31)}$. Other interferences vanish due to zero $b$ or $u$ quark mass.

The full results from these four-fermion operators are: 
$t \rightarrow b e^{+} \nu:$

$$
\frac{\mathrm{d} \Gamma_{b e^{+} \nu}}{\mathrm{d} Q^{2} \mathrm{~d} \cos \theta}=\Gamma_{S+T}\left(x, \cos \theta ; C_{l e Q t}^{(1)}, C_{l e Q t}^{(3)}\right)+\Gamma_{S+T}\left(x, \cos \theta ; C_{l e b Q}, 0\right)
$$

$t \rightarrow u e^{+} e^{-}:$

$$
\frac{\mathrm{d} \Gamma_{u e^{+} e^{-}}}{\mathrm{d} Q^{2} \mathrm{~d} \cos \theta}=\Gamma_{S+T}\left(x, \cos \theta ; C_{\text {lequ }}^{(1,13)}, C_{\text {lequ }}^{(3,13)}\right)+\Gamma_{S+T}\left(x, \cos \theta ; C_{\text {lequ }}^{(1,31) *}, C_{\text {lequ }}^{(3,31) *}\right),
$$

where $x^{2}=Q^{2} / m_{t}^{2}$, and there is no contributions to $t \rightarrow u \nu \bar{\nu}$ because a scalar or a tensor current always involves right-handed neutrino. The function $\Gamma_{S+T}$ is defined as:

$$
\begin{aligned}
\Gamma_{S+T}\left(x, \cos \theta ; c^{(1)}, c^{(3)}\right)= & \frac{1}{128 \pi^{3}} \frac{m_{t}^{3}}{\Lambda^{4}}\left\{\left|c^{(1)}\right|^{2} \gamma_{S S, 0}(x, \cos \theta)\left[1+\alpha_{s} \delta_{S}(x)\right]\right. \\
& \left.+\operatorname{Re}\left(c^{(1)} c^{(3) *}\right) \gamma_{S T, 0}(x, \cos \theta)\left[1+\alpha_{s} \delta_{S T}(x)\right]+\left|c^{(3)}\right|^{2} \sum_{i=1,2} \gamma_{T T, 0}^{(i)}(x, \cos \theta)\left[1+\alpha_{s} \delta_{T T}^{(i)}(x)\right]\right\}
\end{aligned}
$$

where the tree level contributions are given by

$$
\begin{aligned}
\gamma_{S S, 0}(x, \cos \theta) & =\frac{1}{8} x^{2}\left(1-x^{2}\right)^{2}\left[f^{+}(\cos \theta)+f^{0}(\cos \theta)+f^{-}(\cos \theta)\right] \\
\gamma_{S T, 0}(x, \cos \theta) & =-x^{2}\left(1-x^{2}\right)^{2}\left[f^{+}(\cos \theta)-f^{-}(\cos \theta)\right] \\
\gamma_{T T, 0}^{(1)}(x, \cos \theta) & =2 x^{2}\left(1-x^{2}\right)^{2}\left[f^{+}(\cos \theta)-f^{0}(\cos \theta)+f^{-}(\cos \theta)\right] \\
\gamma_{T T, 0}^{(2)}(x, \cos \theta) & =4\left(1-x^{2}\right)^{2}\left[f^{0}(\cos \theta)\right],
\end{aligned}
$$

and the NLO corrections, $\delta_{S, S T}(x)$ and $\delta_{T T}^{(i)}(x)$ are given in Appendix A.

\section{NUMERICAL RESULTS}

In this section we present some numerical results. In particular we focus on the effects of the top-quark color-dipole operator $O_{t G}$ and the four-fermion operators, which are often ignored in previous studies. Nevertheless, we will not go into too much detail. The main purpose of this paper is to provide analytical results, rather than to discuss their phenomenological aspects. A more complete study of their phenomenological implications, including strategies for searching and bounding the operators, will presented elsewhere.

Throughout this section we use the following values as input parameters:

$$
\begin{aligned}
& m_{W}=80.385 \mathrm{GeV} \\
& m_{Z}=91.1876 \mathrm{GeV} \\
& m_{t}=173 \mathrm{GeV} \\
& m_{h}=125 \mathrm{GeV} \\
& G_{F}=1.1663787 \times 10^{-5} \mathrm{GeV}^{-2} \\
& \alpha_{s}\left(m_{t}\right)=0.1081 .
\end{aligned}
$$

The strong coupling $\alpha_{s}\left(m_{t}\right)$ is obtained with RunDec [77] from the value $\alpha_{s}\left(m_{Z}\right)=0.1185$ [78].
A. $t \rightarrow b W$

The main channel decay has been computed in Ref. [56] at NLO and the width and $W$-helicity fractions are given in terms of the anomalous $t b W$ couplings. These anomalous couplings are in one-to-one correspondence with the coefficients of the four operators, $O_{\varphi Q}^{(3)}, O_{\varphi \varphi}, O_{t W}$ and $O_{b W}$. Our results include the contribution from the topquark dipole operator $O_{t G}$, which is a pure NLO effect. To illustrate its numerical impact, here we will focus only on $O_{t W}$ and $O_{t G}$. The other operators are either tightly constrained from $B$ meson decay [79] or do not change the $W$-helicity fractions. For the numerical results we will also include the $m_{b} \neq 0$ effects for the dimension-six operators [50] at LO. We also include the NNLO QCD correction to the SM contribution [53]. The off-shellness and finite-width effect of the $W$ is taken into consider. We assume $\Lambda=1 \mathrm{TeV}$ and both $C_{t W}$ and $C_{t G}$ are real.

Up to order $\alpha_{s} / \Lambda^{2}$, we find the total width:

$$
\begin{aligned}
\Gamma^{t o t} & =\left[1.311+0.158 C_{t W}+\alpha_{s}\left(-0.11 C_{t W}-0.04 C_{t G}\right)\right] \mathrm{GeV} \\
& =\left(1.311+0.146 C_{t W}-0.004 C_{t G}\right) \mathrm{GeV},
\end{aligned}
$$


and the helicity fractions:

$$
\begin{aligned}
F_{0} & =0.689-0.040 C_{t W}+\alpha_{s}\left(0.006 C_{t W}+0.007 C_{t G}\right) \\
& =0.689-0.039 C_{t W}+0.0007 C_{t G} \\
F_{+} & =\left[1.69-0.04 C_{t W}+\alpha_{s}\left(-0.57 C_{t W}+0.31 C_{t G}\right)\right] \times 10^{-3} \\
& =\left[1.69-0.10 C_{t W}+0.03 C_{t G}\right] \times 10^{-3},
\end{aligned}
$$

where $F_{0,+} \equiv \Gamma^{(0,+)} / \Gamma^{(t o t)}$ are the fractions of longitudinal and transverse-positive $W$. The contributions from the non-zero bottom-quark mass are about $-0.4 \%$ for $\Gamma^{t o t},-0.1 \%$ for $F_{0}$ and $25.7 \%$ for $F_{+}$. We have fixed the renormalization scale at $\mu=m_{t}$.

For $\Gamma$ and $F_{0}$ the influence from $C_{t G}$ is very small, as expected. It has the same order of magnitude as the NLO QCD correction to the operator $O_{t W}$. In some sense this effect can be considered as a $\mathcal{O}\left(\Lambda^{-2}\right)$ modification to the standard QCD correction, and will shift the NLO correction to the operator $O_{t W}$.

On the other hand, $F_{+}$is more interesting because, at order $\mathcal{O}\left(\Lambda^{-2}\right)$ and in the $m_{b}=0$ limit, $F_{+}$vanishes at tree level even if anomalous $t b W$ couplings are present. The contributions to $F_{+}$can only come from either a non-zero $m_{b}$ effect or a $\mathcal{O}\left(\alpha_{s}\right)$ real gluon emission. For this reason the contribution from $O_{t G}$ is relatively more important. In addition, the LHC sensitivity on $F_{+}$is better than the other two helicities, at 2 per-mille level for $L=10 \mathrm{fb}^{-1}$ [80]. Our numerical result shows that the contribution from $O_{t G}$ is about $1 / 3$ of that from $O_{t W}$, and is not a negligible effect. Note the $-0.04 C_{t W}$ in the first line of Eq. (189) is a finite $m_{b}$ effect.

In the above results we have chosen $\mu=m_{t}$ as our renormalization scale, and thus the operator coefficients in these results should be interpreted as being defined at a low energy scale $m_{t}$. These coefficients are probed by low energy experiments, but they are not directly related to the new physics, which resides at a higher scale $\Lambda$. In general, by matching the new physics to an effective field theory at scale $\Lambda$, one obtains coefficients of operators, $C_{i}(\Lambda)$, defined at scale $\Lambda$, and they need to be evolved down to the scale $m_{t}$, to be compared with experimental results. The operator evolution of $O_{t W}$ and $O_{t G}$ is given in Section III. In some cases one might be more interested in $C_{i}(\Lambda)$ rather than $C_{i}\left(m_{t}\right)$ because they are directly related to the new physics. For this reason it is also useful to present results in terms of $C_{t W, t G}(\Lambda)$, taking into account the running and mixing of the coefficients. The results then depend on the scale $\Lambda$. Assuming $\Lambda=2$
$\mathrm{TeV}$, the helicity fractions are

$$
\begin{aligned}
F_{0}= & 0.689-0.038 C_{t W}(\Lambda)\left(\frac{1 \mathrm{TeV}}{\Lambda}\right)^{2} \\
& +0.0026 C_{t G}(\Lambda)\left(\frac{1 \mathrm{TeV}}{\Lambda}\right)^{2} \\
F_{+}= & {\left[1.69-0.100 C_{t W}(\Lambda)\left(\frac{1 \mathrm{TeV}}{\Lambda}\right)^{2}\right.} \\
& \left.+0.038 C_{t G}(\Lambda)\left(\frac{1 \mathrm{TeV}}{\Lambda}\right)^{2}\right] \times 10^{-3} .
\end{aligned}
$$

We can see the contribution of $O_{t G}$ in $F_{0}$ is enhanced by a factor of $\sim 4$ due to its mixing into $O_{t W}$. To compare the results at scale $\mu=m_{t}$ and scale $\mu=\Lambda$, we show the contour plots for $F_{0}$ and $F_{+}$at two different scales in Figure 7 .

\section{B. $t \rightarrow b l \nu$}

In this section we discuss the effects of four-fermion operators on $W$-helicity fractions. The $W$-helicity fractions have been measured by both ATLAS and CMS. The results are normally used to set limits on the anomalous $t b W$ couplings, $V_{L, R}$ and $g_{L, R}$, or alternatively on the coefficient of the operator $O_{t W}$ (or $O_{u W}^{33}$ ). However, one can imagine that a four-fermion contact operator involving $t b l \nu$ may also lead to decay with the same $b l \nu$ final state, giving rise to a shift of the measured " $W$-helicity fractions", even though a real $W$ is not involved in the process. One example is that some new heavy particle, $W^{\prime}$, will mediate the decay through $t \rightarrow b W^{* *} \rightarrow b l \nu$. If the mass of $W^{\prime}$ is much larger than $m_{t}$, this process is well described by the four-fermion operator $O_{l Q}^{(3)}$.

The contribution from four-fermion operators are typically small, because their interference with the SM amplitude is suppressed by a small numerical factor due to a cancellation in total rate between two phase space regions, $m_{l \nu}<m_{W}$ and $m_{l \nu}>m_{W}$ (where $m_{l \nu}$ is the invariant mass of the lepton and neutrino) in which the $W$ propagator changes sign [9]. Their squared amplitude do not suffer from this cancellation but are suppressed by an additional factor of $1 / \Lambda^{2}$. However, so far some of these operators can be probed only in the top decay process, and one might hope to bound these operators using the differential decay rate of $t \rightarrow b l \nu$. Furthermore, the invariant mass distribution of the lepton pairs are also sensitive to the contact interaction, and probing the off-shell region of $m_{l \nu}$ may provide some information about the four-fermion operators. Unfortunately, so far experimental measurements on $W$-helicity fractions have required that $m_{l \nu}$ is equal to $m_{W}$, in order to determine the momentum of the neutrino. This condition certainly does not apply to the case where the decay is mediated by a heavy $W^{\prime}$, and so the current limits on the helicity 

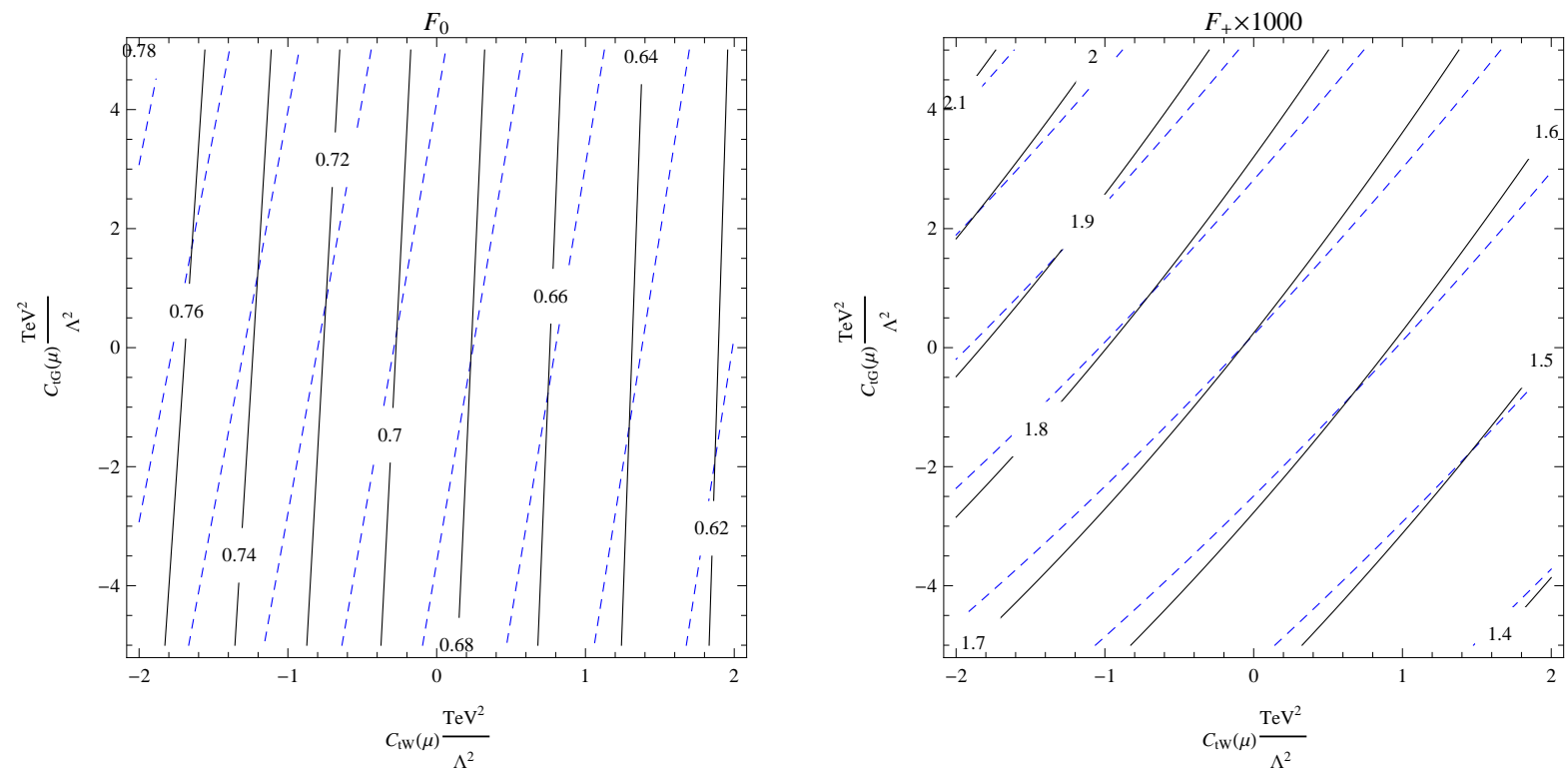

FIG. 7: Contour plots for $W$ helicity fractions in the $C_{t W}(\mu)-C_{t G}(\mu)$ plane. Left: $F_{0}$. Right: $1000 \times F_{+}$. The solid (black) curves represent $\mu=m_{t}$, while the dashed (blue) ones are for $\mu=2 \mathrm{TeV}$.

fractions cannot be used to put limits on four-fermion operators.

The " $W$-helicity fraction" is extracted from the $\cos \theta$ distribution of the decay rate, and in principle this can be done even if a real $W$ boson is not involved, i.e. when a four-fermion operator is present. It is well known that the SM and its interference with two-fermion operator $O_{\varphi Q}^{(3)}$ produces $W$-helicities with the following ratio (at leading order):

$$
F_{+}: F_{0}: F_{-}=0: 1: 2 x^{2},
$$

where $x=m_{W} / m_{t}$, while its interference with twofermion operator $O_{t W}$ gives:

$$
F_{+}: F_{0}: F_{-}=0: 1: 2,
$$

note $F_{+}$is always zero. If the contribution comes from four-fermion operators $O_{l e Q t}^{(1)}$ and $O_{l e b Q}$, which take the form of a scalar-current interaction, then the resulting differential decay rate has no dependence on $\cos \theta$. One would measure

$$
F^{+}: F^{0}: F^{-}=1: 1: 1
$$

from the angular distribution. On the other hand, the operator $O_{\text {leQt }}^{(3)}$ corresponds to a tensor-current interaction, and will give rise to different helicity fractions with the following ratio:

$$
F^{+}: F^{0}: F^{-}=x^{2}: 2-x^{2}: x^{2},
$$

where here $x=m_{l \nu} / m_{t}$ is not fixed at $m_{W} / m_{t}$. The vector-current operator $O_{l Q}^{(3)}$ on the other hand gives rise to a $V-A$ interaction, and so its contribution to the helicity fractions has the same ratio as in the SM, i.e.

$$
F^{+}: F^{0}: F^{-}=0: 1: 2 x^{2} .
$$

Nevertheless the interference between this operator and the SM will cause a shift in the distribution of $m_{l \nu}$, which in turn will modify the helicity fractions.

In Table I we present the numerical results for the helicity fractions, from the SM contribution squared, the interference between the SM and the two-fermion operators $O_{\varphi Q}^{(3)}$ and $O_{t W}$ as well as the four-fermion operator $O_{l Q}^{(3)}$, and the squared contribution from four-fermion operators. We have assumed $\Lambda=1 \mathrm{TeV}$ and all coefficients are equal to one. To present the results in a more useful way, we divide the phase space region into three sub-regions: $15 \mathrm{GeV}<m_{l \nu}<70.4 \mathrm{GeV}, 70.4$ $\mathrm{GeV}<m_{l \nu}<90.4 \mathrm{GeV}$, and $m_{l \nu}>90.4 \mathrm{GeV}$. They are chosen such that the second region corresponds to essentially two-body decay $t \rightarrow b W$ and incorporates most contributions from two-fermion operators. The results given in the table are obtained by integrating over each phase space sub-region. The NLO QCD corrections, the finite $m_{b}$ effect at $\mathrm{LO}$, and the finite $W$-width corrections are all taken into account. The magnitudes of NLO corrections are also given in the table. One can see that in the second sub-region, i.e. the "on-shell" region, the contribution is dominated by the SM and its interference with two-fermion operators, and therefore helicity fractions measured in this region provide information on $t b W$ couplings. On the other hand, in the other two "offshell" regions the SM and two-fermion operators are sup- 
pressed, and four-fermion contributions at order $\Lambda^{-4}$ are comparable with the two-fermion contributions at order $\Lambda^{-2}$. In particular the four-fermion operators will modify the positive helicity fraction which is almost zero in the SM, and this may help to determine or to constrain the coefficient of four-fermion operators.

\section{C. $\quad t \rightarrow u l^{+} l^{-}$}

For flavor-changing decays $t \rightarrow u l^{+} l^{-}$, the situation is very similar. Searches for $t \rightarrow u Z$ has been performed by assuming the lepton pair comes from an on-shell $Z$, and cuts on invariant mass of the leptons near the $Z$-mass shell are applied. In principle, one should consider also the possibility that FCNC is mediated by new heavy particles like $Z^{\prime}$. In this case the decay process is described by four-fermion contact interactions, and so one should take into account their squared contributions as well as their interferences with $t \rightarrow u \gamma^{*}, u Z^{*} \rightarrow u l^{+} l^{-}$. Unlike the main decay channel where the interference between SM and four-fermion operators is suppressed, in the flavor-changing decay mode all contributions start at order $\Lambda^{-4}$, so the four-fermion operators are relatively more important.

To present the decay rate from each operator, we write the decay width of $t \rightarrow u e^{+} e^{-}$as

$$
\Gamma_{t \rightarrow u e^{+} e^{-}}=\sum_{i, j} \Gamma_{i j} C_{i}\left(m_{t}\right) C_{j}\left(m_{t}\right) \frac{(1 \mathrm{TeV})^{2}}{\Lambda^{4}},
$$

where $C_{i}\left(m_{t}\right)$ are either the real or the imaginary part of the operator coefficients, defined at scale $m_{t}$. In Table II we show the numerical values of $\Gamma_{i j}$ for some typical operators, including $O_{\varphi q}^{(1,1+3)}$ which gives rise to the vector coupling of $Z, O_{u W, u G}^{(13)}$ which gives rise to the tensor couplings of $Z, \gamma$ and $g$, and the S-S, V-V and T-T types four-fermion operators, assuming their coefficients are real. These numerical results are obtained with a cut on the invariant mass of the lepton pair, $m_{l l}>15 \mathrm{GeV}$. This is required not only to remove not only the QCD background but also the divergence from the photon peak, which comes from the weak-dipole operators $O_{u B, u W}^{(13),(31)}$. In the table we also show the amount of NLO corrections. The complete results for all operators are given in Appendix B. Note that the interference between S-S and $\mathrm{T}$-T operators is proportional to $\cos \theta$, and vanishes only after integrating over the $\theta$ angle. The QCD correction to this part is about $-2 \%$.

If the flavor-changing decays are observed at the LHC, the next step will be to determine the specific form of the flavor-changing interactions. To this end one needs to make use of the kinematic information of the final state leptons. We have provided results for the differential decay rates, including the invariant-mass distribution and the angular distribution of the final state leptons, and we expect these results will be useful in future analyses for flavor-changing top-quark interactions.

\section{SUMMARY}

Measurements on top-quark related processes can provide valuable information on new physics. In general, non-standard interactions of the top quark should be studied in a global manner, in particular due to their mixing effects at NLO in QCD. For this reason a modelindependent analysis based on an effective field theory approach should be performed, and a global fit needs to be done, including all available measurements and all dimension-six operators.

In this paper we have presented the complete calculation for top-quark semi-leptonic decays in the presence of new physics, at the NLO accuracy in QCD in an EFT approach. We have employed the operator basis in Ref. [63], and consider all dimension-six operators that give rise to a non-standard interaction of the top quark and contribute to top-quark decay processes, including both flavor-conserving and flavor-changing decay modes. Apart from confirming results that were previously available in the literature, we have taken into account the QCD corrections from the color-dipole operators, the differential decay rate of semi-leptonic final states, and the contributions from four-fermion operators.

Our results are presented in terms of analytical expressions for total and differential decay rates as well as their numerical evaluation. The QCD corrections can reach the ten-percent level depending on the processes and operators. In addition, in many cases new contributions enter at the NLO, e.g. from color-dipole operators. For completeness we have also presented the $\mathcal{O}\left(\alpha_{s}\right)$ mixing of all relevant operators.

Our results complete the set of calculations needed for a model-independent study of top-quark decay at NLO accuracy and therefore provide all information needed to perform global analyses for new physics searches.

\section{ACKNOWLEDGEMENTS}

I am grateful for valuable discussions with Gauthier Durieux and Fabio Maltoni. The work is supported by the IISN "Fundamental interactions" convention 4.4517.08. 


\begin{tabular}{|c|c|c|c|c|c|c|c|c|c|c|c|c|c|c|c|}
\hline & $\begin{array}{r}\text { Region } \\
\text { Total }(\mathrm{GeV}) \\
\end{array}$ & $\begin{array}{l}1:[10, \\
\text { NLO }\end{array}$ & $F_{+}$ & $\begin{array}{c}\mathrm{GeV} \\
F_{0} \\
\end{array}$ & $F_{-}$ & $\begin{array}{l}\text { Region } \\
\text { Total }(\mathrm{GeV}) \\
\end{array}$ & $\begin{array}{l}2:[70 . \\
\mathrm{NLO}\end{array}$ & $F_{+}$ & $\begin{array}{l}\mathrm{Ge} \\
F_{0} \\
\end{array}$ & $F_{-}$ & $\begin{array}{r}\text { Region } \\
\text { Total }(\mathrm{GeV}) \\
\end{array}$ & NLO & $F_{+}$ & $F_{0}$ & $F_{-}$ \\
\hline $\mathrm{SM}^{2}$ & $4.2 \times 10^{-3}$ & $-9 \%$ & .00 & 0.80 & 0 & $1.4 \times 10^{-1}$ & $9 \%$ & 0.00 & 0.69 & 0.31 & $3.1 \times 10^{-3}$ & $-9 \%$ & 0.00 & 0.58 & 0.42 \\
\hline $\mathrm{SM} \times C_{\varphi Q}^{(3)}$ & $5.1 \times 10^{-4}$ & $-9 \%$ & 0.00 & 0.80 & 0.20 & $1.7 \times 10^{-2}$ & $-9 \%$ & 0.00 & 0.69 & 0.31 & $3.8 \times 10^{-4}$ & $-9 \%$ & 0.00 & 0.58 & 0.42 \\
\hline $\mathrm{SM} \times C_{t W}$ & 3. $\times 10^{-4}$ & $-8 \%$ & 0.00 & 0.34 & 0.66 & $1.5 \times 10^{-2}$ & $-8 \%$ & 0.00 & 0.34 & 0.66 & & $-8 \%$ & 0.00 & 0.34 & 0.66 \\
\hline $\mathrm{SM} \times C_{l Q}^{(3)}$ & $-4.6 \times 10^{-4}$ & $-9 \%$ & 0.00 & 0.83 & 0.17 & $2.8 \times 10^{-6}$ & $1 \%$ & 0.05 & 8.75 & 9.70 & $4.2 \times 10^{-4}$ & $-9 \%$ & 0.00 & 0.54 & 0.46 \\
\hline$C_{l Q}^{(3)}$ & $1.4 \times 10^{-5}$ & $-9 \%$ & 0.00 & 0.85 & 0.14 & $8.7 \times 10^{-6}$ & $-9 \%$ & 0.00 & 0.69 & 0.31 & $1.9 \times 10^{-5}$ & $-9 \%$ & 0.01 & 0.46 & 0.53 \\
\hline & $1.1 \times 10^{-7}$ & $-0 \%$ & 0.33 & 0.33 & 0.33 & $1.4 \times 10^{-7}$ & $0 \%$ & 0.33 & 0.33 & 0.33 & $5.8 \times 10^{-7}$ & $2 \%$ & 0.33 & 0.33 & 0.33 \\
\hline$C_{l e b Q}^{2}$ & $1.1 \times 10^{-7}$ & $-0 \%$ & 0.33 & 0.33 & 0.33 & $1.4 \times 10^{-7}$ & $0 \%$ & 0.33 & 0.33 & 0.33 & $5.8 \times 10^{-7}$ & $2 \%$ & 0.33 & 0.33 & 0.33 \\
\hline$C_{\text {leQt }}^{(3) 2}$ & $1.3 \times 10^{-5}$ & $-9 \%$ & 0.04 & 0.92 & 0.04 & $6.9 \times 10^{-6}$ & $-9 \%$ & 0.10 & 0.79 & 0.10 & $1.5 \times 10^{-5}$ & $-8 \%$ & 0.19 & 0.62 & 0.19 \\
\hline
\end{tabular}

TABLE I: Numerical values of the helicity fractions at NLO, from the SM contribution, its interference with $O_{\varphi Q}^{(3)}, O_{t W}$ and $O_{l Q}^{(3)}$, and the squared contributions of four-fermion operators. The phase space is divided in to three regions according to $m_{l \nu}$, in such a way that the second sub-region is near the $W$ shell and incorporates almost all "on shell" decays. For each region we present the total width, the NLO correction $\left(\Gamma_{\mathrm{NLO}} / \Gamma_{\mathrm{LO}}-1\right)$, and the three helicity fractions.

\begin{tabular}{|c|c|c|c|c|c|c|c|}
\hline$\Gamma_{i j}(\mathrm{GeV})$ & $\operatorname{Re}\left(C_{\varphi q}^{(1,1+3)}\right)$ & $\operatorname{Re}\left(C_{u W}^{(13)}\right)$ & $\operatorname{Re}\left(C_{u G}^{(13)}\right)$ & $\operatorname{Re}\left(C_{l q}^{(1,1+3)}\right)$ & $\Gamma_{i j}(\mathrm{GeV})$ & $\operatorname{Re}\left(C_{\text {lequ }}^{(1,13)}\right)$ & $\operatorname{Re}\left(C_{\text {lequ }}^{(3,13)}\right)$ \\
\hline $\operatorname{Re}\left(C_{\varphi q}^{(1,1+3)}\right)$ & $1.9 \times 10^{-5}$ & $\begin{array}{c}-6.2 \times 10^{-5} \\
-8 \%\end{array}$ & $2.9 \times 10^{-6}$ & $\begin{array}{c}-3.5 \times 10^{-7} \\
-12 \%\end{array}$ & $\operatorname{Re}\left(C_{\text {lequ }}^{(1,13)}\right)$ & $8.2 \times 10^{-7}$ & 0. \\
\hline $\operatorname{Re}\left(C_{u W}^{(13)}\right)$ & & $\begin{array}{c}7.6 \times 10^{-5} \\
-9 \%\end{array}$ & $-6.1 \times 10^{-6}$ & $\begin{array}{c}-3.3 \times 10^{-6} \\
-7 \%\end{array}$ & $\operatorname{Re}\left(C_{\text {lequ }}^{(3,13)}\right)$ & & $3.5 \times \underset{-8 \%}{\times 10^{-5}}$ \\
\hline $\operatorname{Re}\left(C_{u G}^{(13)}\right)$ & & & $6.8 \times 10^{-8}$ & $2.6 \times 10^{-7}$ & & & \\
\hline $\operatorname{Re}\left(C_{l q}^{(1,1+3)}\right)$ & & & & $2.9 \times \underset{-8 \%}{\times 10^{-6}}$ & & & \\
\hline
\end{tabular}

TABLE II: Numerical values for $\Gamma_{i j}$ from some typical operators. Left: Two-fermion operators and V-V type four-fermion operator, and their interferences. Right: S-S and T-T type four-fermion operators and their interferences. The percentage number under each entry represents the amount of NLO correction (a dash implies the presented value vanishes at LO).

\section{Appendix A: NLO corrections}

In the following we collect all $\mathcal{O}\left(\alpha_{s}\right)$ corrections that appeared in the main text.

The $\delta_{V, V T, T, V G}^{i}(x)$ functions, appeared in Eq. (122-124) and Eq. (140), are:

$$
\begin{aligned}
\delta_{V}^{\text {tot }}(x) & =-\frac{1}{9 \pi\left(x^{2}-1\right)^{2}\left(2 x^{2}+1\right)}\left\{+\left(x^{2}-1\right)\left(3\left(-6 x^{4}+9 x^{2}+5\right)+\pi^{2}\left(8 x^{4}-4 x^{2}-4\right)\right)\right. \\
& +6\left(x^{2}-1\right)^{2}\left(4 x^{2}+5\right) \log (1-x)-24 x^{2}\left(2 x^{4}+x^{2}-1\right) \log (x)+24\left(2 x^{6}-3 x^{4}+1\right) \log (1-x) \log (x) \\
& +6\left(x^{2}-1\right)^{2}\left(4 x^{2}+5\right) \log (x+1)+24\left(2 x^{6}-3 x^{4}+1\right) \log (x) \log (x+1)+48\left(2 x^{6}-3 x^{4}+1\right) \operatorname{Li}_{2}(-x) \\
& \left.+48\left(2 x^{6}-3 x^{4}+1\right) \operatorname{Li}_{2}(x)\right\} \\
\delta_{V T}^{\operatorname{tot}}(x) & =-\frac{1}{9 \pi x^{2}\left(x^{2}-1\right)^{2}}\left\{-3 x^{2}\left(x^{2}-1\right)^{2} \log \left(\frac{m_{t}^{2}}{\mu^{2}}\right)+x^{2}\left(x^{2}-1\right)\left(-21 x^{2}+4 \pi^{2}\left(x^{2}-1\right)+17\right)\right. \\
& +2\left(x^{2}-1\right)^{2}\left(7 x^{2}+2\right) \log (1-x)+8 x^{4}\left(3-2 x^{2}\right) \log (x)+24 x^{2}\left(x^{2}-1\right)^{2} \log (1-x) \log (x) \\
& +2\left(x^{2}-1\right)^{2}\left(7 x^{2}+2\right) \log (x+1)+24 x^{2}\left(x^{2}-1\right)^{2} \log (x) \log (x+1)+48 x^{2}\left(x^{2}-1\right)^{2} \operatorname{Li}_{2}(-x) \\
& \left.+48 x^{2}\left(x^{2}-1\right)^{2} \operatorname{Li}_{2}(x)\right\}
\end{aligned}
$$




$$
\begin{aligned}
\delta_{T}^{\text {tot }}(x) & =-\frac{1}{9 \pi\left(x^{2}-1\right)^{2}\left(x^{2}+2\right)}\left\{-6\left(x^{6}-3 x^{2}+2\right) \log \left(\frac{m_{t}^{2}}{\mu^{2}}\right)\right. \\
& +\left(x^{2}-1\right)\left(-7 x^{4}-13 x^{2}+4 \pi^{2}\left(x^{4}+x^{2}-2\right)+32\right)+6\left(x^{2}-1\right)^{2}\left(x^{2}+8\right) \log (1-x) \\
& -24 x^{2}\left(x^{4}+2 x^{2}-2\right) \log (x)+24\left(x^{6}-3 x^{2}+2\right) \log (1-x) \log (x)+6\left(x^{2}-1\right)^{2}\left(x^{2}+8\right) \log (x+1) \\
& \left.+24\left(x^{6}-3 x^{2}+2\right) \log (x) \log (x+1)+48\left(x^{6}-3 x^{2}+2\right) \operatorname{Li}_{2}(-x)+48\left(x^{6}-3 x^{2}+2\right) \operatorname{Li}_{2}(x)\right\} \\
\delta_{V G}^{\text {tot }}(x) & =\frac{1}{18 \pi x^{2}\left(x^{2}-1\right)^{2}}\left\{+6 x^{2}\left(x^{2}-1\right)^{2} \log \left(\frac{m_{t}^{2}}{\mu^{2}}\right)-1-10 x^{2}+31 x^{4}-20 x^{6}+4\left(x^{2}-1\right)^{3} \log (1-x)\right. \\
& \left.+4 x^{4}\left(x^{2}+3\right) \log (x)+4\left(x^{2}-1\right)^{3} \log (x+1)\right\} \\
\delta_{V}^{L}(x) & =-\frac{1}{9 \pi\left(x^{2}-1\right)^{2}}\left\{+4 \pi^{2}\left(2 x^{4}+5 x^{2}+1\right)-3\left(4 x^{6}-51 x^{4}+42 x^{2}+5\right)+18\left(x^{2}-1\right)^{2} \log (1-x)\right. \\
& -96\left(2 x^{4}+x^{2}\right) \log (x)+12(x-1)^{2}\left(x^{3}+6 x^{2}-x+2\right) \log (1-x) \log (x)+18\left(x^{2}-1\right)^{2} \log (x+1) \\
& -12(x+1)^{2}\left(x^{3}-6 x^{2}-x-2\right) \log (x) \log (x+1)-12(x+1)^{2}\left(x^{3}-8 x^{2}+3 x-4\right) \operatorname{Li}_{2}(-x) \\
& \left.+12(x-1)^{2}\left(x^{3}+8 x^{2}+3 x+4\right) \operatorname{Li} i_{2}(x)\right\}
\end{aligned}
$$

$$
\begin{aligned}
\delta_{V T}^{L}(x) & =-\frac{1}{9 \pi x^{2}\left(x^{2}-1\right)^{2}}\left\{-3 x^{2}\left(x^{2}-1\right)^{2} \log \left(\frac{m_{t}^{2}}{\mu^{2}}\right)+x^{2}\left(-33 x^{4}+30 x^{2}+2 \pi^{2}\left(2 x^{4}-7 x^{2}+1\right)+3\right)\right. \\
& +6\left(2 x^{6}-3 x^{4}+1\right) \log (1-x)-12 x^{4}\left(x^{2}-7\right) \log (x)+12(x-1)^{2} x^{2}\left(2 x^{2}+5 x+1\right) \log (1-x) \log (x) \\
& +6\left(2 x^{6}-3 x^{4}+1\right) \log (x+1)+12 x^{2}(x+1)^{2}\left(2 x^{2}-5 x+1\right) \log (x) \log (x+1) \\
& \left.+12 x^{2}(x+1)^{2}\left(4 x^{2}-9 x+3\right) \operatorname{Li}_{2}(-x)+12(x-1)^{2} x^{2}\left(4 x^{2}+9 x+3\right) \operatorname{Li}_{2}(x)\right\}
\end{aligned}
$$

$$
\begin{aligned}
\delta_{T}^{L}(x) & =-\frac{1}{9 \pi x^{2}\left(x^{2}-1\right)^{2}}\left\{-6 x^{2}\left(x^{2}-1\right)^{2} \log \left(\frac{m_{t}^{2}}{\mu^{2}}\right)+x^{2}\left(3\left(x^{4}-22 x^{2}+21\right)+4 \pi^{2}\left(x^{4}-2 x^{2}-3\right)\right)\right. \\
& +6\left(x^{6}-3 x^{2}+2\right) \log (1-x)-24 x^{2}\left(x^{4}-3 x^{2}-3\right) \log (x)+12(x-1)^{2} x\left(2 x^{3}+3 x^{2}+3\right) \log (1-x) \log (x) \\
& +6\left(x^{6}-3 x^{2}+2\right) \log (x+1)+12 x(x+1)^{2}\left(2 x^{3}-3 x^{2}-3\right) \log (x) \log (x+1) \\
& \left.+12 x(x+1)^{2}\left(4 x^{3}-7 x^{2}+2 x-3\right) \operatorname{Li}_{2}(-x)+12(x-1)^{2} x\left(4 x^{3}+7 x^{2}+2 x+3\right) \operatorname{Li}_{2}(x)\right\} \\
& \delta_{V G}^{L}(x)=\frac{1}{18 \pi x^{2}\left(x^{2}-1\right)^{2}}\left\{+6 x^{2}\left(x^{2}-1\right)^{2} \log \left(\frac{m_{t}^{2}}{\mu^{2}}\right)-3+2\left(\pi^{2}-12\right) x^{2}+\left(33+6 \pi^{2}\right) x^{4}-6 x^{6}\right. \\
& +6\left(x^{2}-1\right)^{3} \log (1-x)-48 x^{4} \log (x)-12(x-1)^{3} x^{2} \log (1-x) \log (x)+6\left(x^{2}-1\right)^{3} \log (x+1) \\
& \left.+12 x^{2}(x+1)^{3} \log (x) \log (x+1)+12 x^{2}(x+1)^{3} \operatorname{Li}_{2}(-x)-12(x-1)^{3} x^{2} \operatorname{Li}_{2}(x)\right\}
\end{aligned}
$$




$$
\begin{aligned}
& \delta_{V}^{T}(x)=-\frac{1}{9 \pi x^{2}\left(x^{2}-1\right)^{2}}\left\{+x^{2}\left(2 \pi^{2}\left(2 x^{4}-5 x^{2}-5\right)-3\left(x^{4}+18 x^{2}-19\right)\right)+6\left(2 x^{6}-3 x^{4}+1\right) \log (1-x)\right. \\
& +12 x^{2}\left(-2 x^{4}+7 x^{2}+5\right) \log (x)+6(x-1)^{2} x\left(4 x^{3}+7 x^{2}+5\right) \log (1-x) \log (x)+6\left(2 x^{6}-3 x^{4}+1\right) \log (x+1) \\
& +6 x(x+1)^{2}\left(4 x^{3}-7 x^{2}-5\right) \log (x) \log (x+1)+6 x(x+1)^{2}\left(8 x^{3}-15 x^{2}+4 x-5\right) \operatorname{Li}_{2}(-x) \\
& \left.+6(x-1)^{2} x\left(8 x^{3}+15 x^{2}+4 x+5\right) \operatorname{Li}_{2}(x)\right\} \\
& \delta_{V T}^{T}(x)=-\frac{1}{9 \pi x^{2}\left(x^{2}-1\right)^{2}}\left\{-3 x^{2}\left(x^{2}-1\right)^{2} \log \left(\frac{m_{t}^{2}}{\mu^{2}}\right)+x^{2}\left(\pi^{2}\left(4 x^{4}-5 x^{2}+5\right)-3\left(5 x^{4}-14 x^{2}+9\right)\right)\right. \\
& +3\left(x^{2}-1\right)^{2}\left(5 x^{2}+1\right) \log (1-x)-6\left(3 x^{6}+x^{4}\right) \log (x)+6(x-1)^{2} x^{2}\left(4 x^{2}+7 x+5\right) \log (1-x) \log (x) \\
& +3\left(x^{2}-1\right)^{2}\left(5 x^{2}+1\right) \log (x+1)+6 x^{2}(x+1)^{2}\left(4 x^{2}-7 x+5\right) \log (x) \log (x+1) \\
& \left.+6 x^{2}(x+1)^{2}\left(8 x^{2}-15 x+9\right) \operatorname{Li}_{2}(-x)+6(x-1)^{2} x^{2}\left(8 x^{2}+15 x+9\right) \operatorname{Li}_{2}(x)\right\} \\
& \delta_{T}^{T}(x)=-\frac{1}{9 \pi\left(x^{2}-1\right)^{2}}\left\{-6\left(x^{2}-1\right)^{2} \log \left(\frac{m_{t}^{2}}{\mu^{2}}\right)-16-9 x^{2}+30 x^{4}-5 x^{6}+4 \pi^{2}\left(x^{4}+1\right)\right. \\
& +18\left(x^{2}-1\right)^{2} \log (1-x)-12\left(5 x^{4}+x^{2}\right) \log (x)+6(x-1)^{2}\left(x^{3}+6 x^{2}+5 x+4\right) \log (1-x) \log (x) \\
& +18\left(x^{2}-1\right)^{2} \log (x+1)+6\left(-x^{5}+4 x^{4}+6 x^{3}+3 x+4\right) \log (x) \log (x+1) \\
& \left.-6(x+1)^{2}\left(x^{3}-10 x^{2}+13 x-8\right) \operatorname{Li}_{2}(-x)+6(x-1)^{2}\left(x^{3}+10 x^{2}+13 x+8\right) \operatorname{Li}_{2}(x)\right\} \\
& \delta_{V G}^{T}(x)=-\frac{1}{18 \pi x^{2}\left(x^{2}-1\right)^{2}}\left\{-6 x^{2}\left(x^{2}-1\right)^{2} \log \left(\frac{m_{t}^{2}}{\mu^{2}}\right)+x^{2}\left(27 x^{4}-30 x^{2}+\pi^{2}\left(3 x^{2}+1\right)+3\right)\right. \\
& -3\left(x^{2}-1\right)^{3} \log (1-x)-6 x^{4}\left(x^{2}+7\right) \log (x)-6(x-1)^{3} x^{2} \log (1-x) \log (x)-3\left(x^{2}-1\right)^{3} \log (x+1) \\
& \left.+6 x^{2}(x+1)^{3} \log (x) \log (x+1)+6 x^{2}(x+1)^{3} \operatorname{Li}_{2}(-x)-6(x-1)^{3} x^{2} \operatorname{Li}_{2}(x)\right\} \\
& \delta_{V}^{F}(x)=-\frac{2}{9 \pi x^{2}\left(x^{2}-1\right)^{2}}\left\{+x^{2}\left(\pi^{2}\left(x^{2}+2\right)+3(4 x-3)(x-1)^{2}\right)+\left(6 x^{6}-9 x^{4}+3\right) \log (1-x)\right. \\
& \left.+\left(-6 x^{6}+33 x^{4}-30 x^{2}+3\right) \log (x+1)-12 x^{2}\left(x^{4}-3 x^{2}-1\right) \operatorname{Li}_{2}(-x)+12 x^{2}\left(x^{2}-1\right)^{2} \operatorname{Li}_{2}(x)\right\} \\
& \delta_{V T}^{F}(x)=-\frac{1}{9 \pi x^{2}\left(x^{2}-1\right)^{2}}\left\{-3 x^{2}\left(x^{2}-1\right)^{2} \log \left(\frac{m_{t}^{2}}{\mu^{2}}\right)+x^{2}\left(5 \pi^{2}\left(x^{2}+1\right)-3(x-1)^{2}\left(5 x^{2}-12 x+9\right)\right)\right. \\
& +3\left(x^{2}-1\right)^{2}\left(5 x^{2}+1\right) \log (1-x)+\left(-3 x^{6}+69 x^{4}-69 x^{2}+3\right) \log (x+1) \\
& \left.+12 x^{2}\left(-2 x^{4}+9 x^{2}+3\right) \operatorname{Li}_{2}(-x)+24 x^{2}\left(x^{2}-1\right)^{2} \operatorname{Li}_{2}(x)\right\} \\
& \delta_{T}^{F}(x)=\frac{1}{9 \pi\left(x^{2}-1\right)^{2}}\left\{+6\left(x^{2}-1\right)^{2} \log \left(\frac{m_{t}^{2}}{\mu^{2}}\right)+2 \pi^{2}\left(x^{4}-2\right)+(x-1)^{2}\left(x^{4}+2 x^{3}-9 x^{2}-28 x+16\right)\right. \\
& \left.-18\left(x^{2}-1\right)^{2} \log (1-x)+6\left(5 x^{4}-12 x^{2}+7\right) \log (x+1)+24\left(2 x^{4}-2 x^{2}-1\right) \operatorname{Li}_{2}(-x)-24\left(x^{2}-1\right)^{2} \operatorname{Li}_{2}(x)\right\}
\end{aligned}
$$




$$
\begin{aligned}
\delta_{V G}^{F}(x) & =\frac{1}{18 \pi x^{2}\left(x^{2}-1\right)^{2}}\left\{+6 x^{2}\left(x^{2}-1\right)^{2} \log \left(\frac{m_{t}^{2}}{\mu^{2}}\right)+x^{2}\left(\pi^{2}\left(3 x^{2}+1\right)-6(x-1)^{2}\left(5 x^{2}+3 x+4\right)\right)\right. \\
& \left.+3\left(x^{2}-1\right)^{3} \log (1-x)+3\left(3 x^{6}+x^{4}-3 x^{2}-1\right) \log (x+1)+12\left(3 x^{4}+x^{2}\right) \operatorname{Li}_{2}(-x)\right\} .
\end{aligned}
$$

In the following we define

$$
y \equiv \frac{x+i \sqrt{4-x^{2}}}{2} .
$$

The $\delta_{V G, T G, G 2}^{i}(x)$ functions that appeared in Eq. (140) are:

$$
\begin{aligned}
& \delta_{V G, r}^{t o t}(x, v, a)=\frac{1}{54 \pi x^{2}\left(x^{2}-1\right)^{2}}\left\{+36 v x^{2}\left(x^{2}-1\right)^{2} \log \left(\frac{m_{t}^{2}}{\mu^{2}}\right)+v x^{2}\left(-81 x^{4}+156 x^{2}-6 \pi \sqrt{4-x^{2}} x^{3}+4 \pi^{2}-75\right)\right. \\
& +a\left(-39 x^{6}+66 x^{4}+\left(4 \pi^{2}-21\right) x^{2}-6 \pi \sqrt{4-x^{2}} x^{5}+18 \pi \sqrt{4-x^{2}} x^{3}-6\right) \\
& +12 x \sin ^{-1}\left(\frac{x}{2}\right)\left(a\left(\sqrt{4-x^{2}}\left(x^{4}-3 x^{2}-4\right)-2 \pi x\right)+v\left(\sqrt{4-x^{2}}\left(7 x^{4}-6 x^{2}+2\right)-2 \pi x\right)\right) \\
& -24 x^{2}(a+v) \sin ^{-1}\left(\frac{x}{2}\right)^{2}-12 x^{2} \log (x)\left(a\left(x^{4}-5 x^{2}-3\right)+v\left(x^{4}+4 x^{2}-3\right)\right) \\
& \left.+24 x^{2}(a+v) \operatorname{ReLi}_{2}(x y)\right\} \\
& \delta_{V G, i}^{t o t}(x, v, a)=-\frac{1}{3}(v+a) \\
& \delta_{T G, r}^{t o t}(x, v, a)=\frac{1}{9 \pi x\left(x^{2}-1\right)^{2}\left(x^{2}+2\right)}\left\{+6 v x\left(x^{6}-3 x^{2}+2\right) \log \left(\frac{m_{t}^{2}}{\mu^{2}}\right)\right. \\
& +x\left[a\left(-3 \pi x \sqrt{4-x^{2}}\left(x^{2}-2\right)+3\left(x^{4}+14 x^{2}-15\right)+4 \pi^{2}\right)\right. \\
& \left.+v\left(-11 x^{6}-12 x^{4}+81 x^{2}-6 \pi \sqrt{4-x^{2}}\left(x^{2}-1\right) x+4 \pi^{2}-58\right)\right] \\
& -6 \sin ^{-1}\left(\frac{x}{2}\right)\left(a\left(\sqrt{4-x^{2}}\left(x^{6}-5 x^{4}+3 x^{2}+4\right)+4 \pi x\right)+v x\left(4 \pi-x \sqrt{4-x^{2}}\left(x^{4}+8 x^{2}-9\right)\right)\right) \\
& \left.-24 x(a+v) \sin ^{-1}\left(\frac{x}{2}\right)^{2}+6 x^{3} \log (x)\left(a\left(x^{4}-7 x^{2}-7\right)+v\left(x^{4}-6 x^{2}-3\right)\right)+24 x(a+v) \operatorname{ReLi}_{2}(x y)\right\} \\
& \delta_{T G, i}^{t o t}(x, v, a)=-\frac{1}{3}(v+a) \\
& \delta_{G 2}^{t o t}(x, v, a)=-\frac{1}{36 \pi x^{2}\left(x^{2}-4\right)\left(x^{2}-1\right)^{2}\left(x^{2}+2\right)}\left\{-a^{2}\left(x^{2}-4\right)\left(5 x^{8}-43 x^{6}+108 x^{4}-\left(71+2 \pi^{2}\right) x^{2}\right.\right. \\
& \left.-9 \pi \sqrt{4-x^{2}} x^{5}+60 \pi \sqrt{4-x^{2}} x^{3}+1\right)-2 a v x^{2}\left(x^{2}-4\right)\left(45 x^{4}-66 x^{2}+3 \pi \sqrt{4-x^{2}}\left(x^{2}+2\right) x-2 \pi^{2}+21\right) \\
& +v^{2} x^{2}\left(-4 x^{8}+19 x^{6}+66 x^{4}-389 x^{2}+2 \pi^{2}\left(x^{2}-4\right)+3 \pi \sqrt{4-x^{2}}\left(3 x^{4}-10 x^{2}-20\right) x+308\right) \\
& -6 x^{2} \sin ^{-1}\left(\frac{x}{2}\right)\left(a^{2}\left(x^{2}-4\right)\left(3 x \sqrt{4-x^{2}}\left(3 x^{2}-20\right)+4 \pi\right)+2 a v\left(x^{2}-4\right)\left(4 \pi-3 x \sqrt{4-x^{2}}\left(x^{2}+2\right)\right)\right. \\
& \left.+v^{2}\left(4 \pi\left(x^{2}-4\right)+3 x \sqrt{4-x^{2}}\left(3 x^{4}-10 x^{2}-20\right)\right)\right) \\
& +72 x^{2}\left(x^{2}-4\right)(a+v)^{2} \sin ^{-1}\left(\frac{x}{2}\right)^{2}+6 x^{2}\left(x^{2}-4\right) \log (x)\left(a^{2}\left(9 x^{4}-46 x^{2}-2\right)+2 a v\left(5 x^{4}-6\right)\right. \\
& \left.\left.+v^{2}\left(9 x^{4}+4 x^{2}-10\right)\right)-24 x^{2}\left(x^{2}-4\right)(a+v)^{2} \log ^{2}(x)\right\}
\end{aligned}
$$




$$
\begin{aligned}
\delta_{V G, r}^{L}(x, v, a) & =-\frac{1}{9 \pi x^{2}\left(x^{2}-1\right)^{3}}\left\{-6 v x^{2}\left(x^{2}-1\right)^{3} \log \left(\frac{m_{t}^{2}}{\mu^{2}}\right)+60 x^{2}(a+v) \sin ^{-1}\left(\frac{x}{2}\right)^{2}\right. \\
& +a\left(3 x^{8}+3 \pi^{2} x^{7}-3\left(3+2 \pi^{2}\right) x^{6}+2\left(3+\pi^{2}\right) x^{4}-3 \pi^{2} x^{3}+3\left(1+\pi^{2}\right) x^{2}-3\right) \\
& +v x^{2}\left(3\left(4 x^{2}-5\right)\left(x^{2}-1\right)^{2}+3 \pi x \sqrt{4-x^{2}}\left(x^{2}-1\right)+\pi^{2}\left(3 x^{5}-6 x^{4}+2 x^{2}-3 x+3\right)\right) \\
& -6 x \sqrt{4-x^{2}}\left(x^{2}-1\right) \sin ^{-1}\left(\frac{x}{2}\right)\left(v\left(x^{4}+2 x^{2}+2\right)-a x^{2}\left(x^{2}-3\right)\right) \\
& -6 x^{2}\left(x^{2}-1\right) \log (x)\left(a\left(x^{4}-4 x^{2}+1\right)+v\left(x^{4}-7 x^{2}+1\right)\right) \\
& -6(x-1)^{3} x\left(x^{3}+3 x^{2}+x-1\right)(a+v) \log (1-x) \log (x)-12 x^{4}\left(x^{2}-1\right)(a+v) \log ^{2}(x) \\
& +6 x(x+1)^{3}\left(x^{3}-3 x^{2}+x+1\right)(a+v) \log (x) \log (x+1)-24 x^{2}(a+v) \operatorname{ReLi}_{2}(x y) \\
& \left.+6 x(x+1)^{3}\left(x^{3}-3 x^{2}+x+1\right)(a+v) \operatorname{Li}(-x)-6(x-1)^{3} x\left(x^{3}+3 x^{2}+x-1\right)(a+v) \operatorname{Li}_{2}(x)\right\}
\end{aligned}
$$

$$
\delta_{V G, i}^{L}(x, v, a)=-\frac{1}{3}(v+a)
$$

$$
\begin{aligned}
\delta_{T G, r}^{L}(x, v, a)= & -\frac{1}{9 \pi x^{3}\left(x^{2}-4\right)\left(x^{2}-1\right)^{3}}\left\{-6 v x^{3}\left(x^{2}-4\right)\left(x^{2}-1\right)^{3} \log \left(\frac{m_{t}^{2}}{\mu^{2}}\right)+24 x\left(3 v-a x^{2}\left(x^{2}-4\right)\right) \operatorname{ReLi}_{2}(x y)\right. \\
+ & x\left(a\left(x^{2}-4\right)\left(\left(2 x^{4}-40 x^{2}-31\right)\left(x^{2}-1\right)^{2}+\pi^{2}\left(2 x^{6}-12 x^{5}-4 x^{4}+12 x^{3}-3 x^{2}+4\right)\right)\right. \\
& \quad+v x\left(12 x^{9}+\left(4 \pi^{2}-69\right) x^{7}+\left(90-8 \pi^{2}\right) x^{5}-3\left(7+15 \pi^{2}\right) x^{3}+3 \pi\left(11 \sqrt{4-x^{2}}-16 \pi\right) x^{2}\right. \\
& \left.\left.\quad-18 \pi \sqrt{4-x^{2}}+3 \pi\left(\sqrt{4-x^{2}}-4 \pi\right) x^{6}+6 \pi\left(10 \pi-3 \sqrt{4-x^{2}}\right) x^{4}+4\left(13 \pi^{2}-3\right) x\right)\right) \\
+ & 6 x\left(x^{2}-1\right) \sin ^{-1}\left(\frac{x}{2}\right)\left(x \sqrt{4-x^{2}}\left(a x^{2}\left(x^{4}-7 x^{2}+12\right)-v\left(x^{6}-2 x^{4}-9 x^{2}+10\right)\right)+4 \pi v\left(x^{2}-3\right)\right) \\
+ & 12 \sin ^{-1}\left(\frac{x}{2}\right)^{2}\left(5 a x^{3}\left(x^{2}-4\right)-3 v x\left(x^{4}-4 x^{2}+8\right)\right)+12 x^{3}\left(x^{6}-4 x^{4}-x^{2}+4\right)(a+v) \log ^{2}(x) \\
- & 6 x\left(x^{4}-5 x^{2}+4\right) \log (x)\left(a\left(x^{6}-8 x^{4}-15 x^{2}-2\right)+v\left(x^{4}-x^{2}+6\right) x^{2}\right) \\
- & 6(x-1)^{3}\left(x^{2}+3 x+2\right) \log (1-x) \log (x)\left(a\left(4 x^{4}-9 x^{3}+4 x^{2}-5 x+2\right)+v x\left(2 x^{3}-x^{2}-8 x+3\right)\right) \\
- & 6(x+1)^{3}\left(x^{2}-3 x+2\right) \log (x) \log (x+1)\left(a\left(4 x^{4}+9 x^{3}+4 x^{2}+5 x+2\right)+v x\left(2 x^{3}+x^{2}-8 x-3\right)\right) \\
- & 6(x+1)^{3}\left(x^{2}-3 x+2\right) \operatorname{Li}(-x)\left(a\left(4 x^{4}+9 x^{3}+4 x^{2}+5 x+2\right)+v x\left(2 x^{3}+x^{2}-8 x-3\right)\right) \\
- & \left.6(x-1)^{3}\left(x^{2}+3 x+2\right) \operatorname{Li}(x)\left(a\left(4 x^{4}-9 x^{3}+4 x^{2}-5 x+2\right)+v x\left(2 x^{3}-x^{2}-8 x+3\right)\right)\right\}
\end{aligned}
$$

$$
\delta_{T G, i}^{L}(x, v, a)=-\frac{1}{3}(v+a)
$$




$$
\begin{aligned}
\delta_{G 2}^{L}(x, v, a)= & -\frac{1}{36 \pi(x-2) x^{4}(x+2)\left(x^{2}-1\right)^{2}}\left\{-a^{2}\left(x^{2}-4\right)\left(x^{8}-28 x^{6}-15 \pi^{2} x^{5}+6\left(\pi^{2}-3\right) x^{4}-15 \pi^{2} x^{3}+44 x^{2}+1\right)\right. \\
& -v^{2} x^{2}\left(6 \pi\left(10-3 x^{2}\right) \sqrt{4-x^{2}} x+12\left(x^{4}-5 x^{2}+4\right) x^{2}+\pi^{2}\left(5 x^{5}-2 x^{4}+5 x^{3}+8 x^{2}-80 x+16\right)\right) \\
& +2 a v x^{2}\left(-6 \pi x \sqrt{4-x^{2}}\left(x^{2}-6\right)+12\left(x^{6}-7 x^{4}+14 x^{2}-8\right)+\pi^{2}\left(5 x^{5}-2 x^{4}-25 x^{3}+8 x^{2}+10 x-8\right)\right) \\
& +12 v x^{2} \sin ^{-1}\left(\frac{x}{2}\right)\left(a\left(6 x \sqrt{4-x^{2}}\left(x^{2}-6\right)+8 \pi\right)+v\left(3 x \sqrt{4-x^{2}}\left(10-3 x^{2}\right)+8 \pi\right)\right) \\
& +96 v x^{2}(a+v) \sin ^{-1}\left(\frac{x}{2}\right)^{2}-12 x^{2}\left(x^{2}-4\right) \log (x)\left(2 a^{2}\left(5 x^{2}+1\right)+2 a v\left(5 x^{2}-2\right)-v^{2}\left(7 x^{2}+2\right)\right) \\
& -24(x-1)^{2} x^{2}(x+2) \log (1-x) \log (x)\left(3 a^{2}(x-2) x+2 a v\left(x^{2}-2 x-2\right)-v^{2}\left(x^{2}-2 x+4\right)\right) \\
& +6 x^{2}(x+2) \log ^{2}(x)\left(3 a^{2} x\left(x^{3}-8 x^{2}+13 x-2\right)\right. \\
& \left.+2 a v\left(x^{4}-8 x^{3}+11 x^{2}+2 x-2\right)-v^{2}\left(x^{4}-8 x^{3}+17 x^{2}-10 x+4\right)\right) \\
& +24(x-2) x^{2}(x+1)^{2} \log (x) \log (x+1)\left(3 a^{2} x(x+2)+2 a v\left(x^{2}+2 x-2\right)-v^{2}\left(x^{2}+2 x+4\right)\right) \\
& +24(x-2) x^{2}(x+1)^{2} \operatorname{Li}_{2}(-x)\left(3 a^{2} x(x+2)+2 a v\left(x^{2}+2 x-2\right)-v^{2}\left(x^{2}+2 x+4\right)\right) \\
& -12(x-1)^{2} x^{2}(x+2) \operatorname{Li}_{2}(x)\left(3 a^{2}(x-2) x+2 a v\left(x^{2}-2 x-2\right)-v^{2}\left(x^{2}-2 x+4\right)\right) \\
& \left.-384 v x^{2}(a+v) \operatorname{ReLi}_{2}(x y)\right\}
\end{aligned}
$$

$$
\begin{aligned}
\delta_{V G, r}^{T}(x, v, a)= & \frac{1}{36 \pi x\left(x^{2}-1\right)^{3}}\left\{+24 v x\left(x^{2}-1\right)^{3} \log \left(\frac{m_{t}^{2}}{\mu^{2}}\right)+24 x\left(x^{2}-3\right)(a+v) \operatorname{ReLi}_{2}(x y)\right. \\
+ & x\left(a\left(-3\left(11 x^{2}-7\right)\left(x^{2}-1\right)^{2}-6 \pi x \sqrt{4-x^{2}}\left(x^{4}-4 x^{2}+3\right)+2 \pi^{2}\left(3 x^{5}-6 x^{4}+4 x^{2}-3 x+1\right)\right)\right. \\
& \left.\quad+v\left(-3\left(19 x^{2}-15\right)\left(x^{2}-1\right)^{2}-6 \pi x \sqrt{4-x^{2}}\left(x^{2}-1\right)^{2}+2 \pi^{2}\left(3 x^{5}-6 x^{4}+4 x^{2}-3 x+1\right)\right)\right) \\
+ & 24\left(x^{2}-1\right) \sin ^{-1}\left(\frac{x}{2}\right)\left(a\left(\sqrt{4-x^{2}}\left(x^{4}-3 x^{2}-2\right)-\pi x\right)+v x\left(x \sqrt{4-x^{2}}\left(3 x^{2}-4\right)-\pi\right)\right) \\
- & 24 x\left(x^{2}-6\right)(a+v) \sin ^{-1}\left(\frac{x}{2}\right)^{2}-24 x^{3}\left(x^{2}-1\right)(a+v) \log ^{2}(x) \\
- & 12 x\left(x^{2}-1\right) \log (x)\left(a\left(2 x^{4}-9 x^{2}-2\right)+v\left(2 x^{4}-3 x^{2}-2\right)\right) \\
- & 12(x-1)^{3}\left(x^{3}+3 x^{2}+x-1\right)(a+v) \log (1-x) \log (x) \\
+ & 12(x+1)^{3}\left(x^{3}-3 x^{2}+x+1\right)(a+v) \log (x) \log (x+1) \\
+ & \left.12(x+1)^{3}\left(x^{3}-3 x^{2}+x+1\right)(a+v) \operatorname{Li} 2(-x)-12(x-1)^{3}\left(x^{3}+3 x^{2}+x-1\right)(a+v) \operatorname{Li}_{2}(x)\right\}\left(\mathrm{A}_{2}\right)
\end{aligned}
$$

$$
\delta_{V G, i}^{T}(x, v, a)=-\frac{1}{3}(v+a)
$$




$$
\begin{aligned}
& \delta_{T G, r}^{T}(x, v, a)=\frac{1}{18 \pi x\left(x^{2}-4\right)\left(x^{2}-1\right)^{3}}\left\{+12 v x\left(x^{2}-4\right)\left(x^{2}-1\right)^{3} \log \left(\frac{m_{t}^{2}}{\mu^{2}}\right)\right. \\
& +x\left(a ( x ^ { 2 } - 4 ) \left(2 x^{8}+\left(2 \pi^{2}-41\right) x^{6}+\left(90-4 \pi^{2}\right) x^{4}+\left(\pi^{2}-65\right) x^{2}-6 \pi \sqrt{4-x^{2}} x\right.\right. \\
& \left.-3 \pi\left(\sqrt{4-x^{2}}+4 \pi\right) x^{5}+3 \pi\left(3 \sqrt{4-x^{2}}+4 \pi\right) x^{3}+14\right) \\
& +v\left(\left(x^{6}-24 x^{4}+138 x^{2}-232\right)\left(x^{2}-1\right)^{2}-3 \pi x \sqrt{4-x^{2}}\left(x^{6}-6 x^{4}+7 x^{2}-2\right)\right. \\
& \left.\left.+\pi^{2}\left(4 x^{8}-12 x^{7}-8 x^{6}+60 x^{5}-41 x^{4}-48 x^{3}+32 x^{2}+16\right)\right)\right) \\
& +6\left(x^{2}-1\right) \sin ^{-1}\left(\frac{x}{2}\right)\left(a\left(x^{2}-4\right)\left(\sqrt{4-x^{2}}\left(2 x^{4}-3 x^{2}-4\right)-4 \pi x\right)\right. \\
& \left.+2 v x\left(x \sqrt{4-x^{2}}\left(3 x^{4}-16 x^{2}+13\right)+2 \pi\right)\right) \\
& +12 x \sin ^{-1}\left(\frac{x}{2}\right)^{2}\left(a\left(3 x^{4}-10 x^{2}-8\right)+v\left(-5 x^{4}+22 x^{2}-32\right)\right) \\
& +6 x\left(x^{4}-5 x^{2}+4\right) \log (x)\left(a\left(x^{4}+8 x^{2}+2\right)-v x^{2}\left(5 x^{2}+9\right)\right) \\
& -6(x-1)^{3}\left(x^{2}+3 x+2\right) \log (1-x) \log (x)\left(a\left(4 x^{4}-9 x^{3}+4 x^{2}-5 x+2\right)+v x\left(2 x^{3}-x^{2}-8 x+3\right)\right) \\
& -6(x+1)^{3}\left(x^{2}-3 x+2\right) \log (x) \log (x+1)\left(a\left(4 x^{4}+9 x^{3}+4 x^{2}+5 x+2\right)+v x\left(2 x^{3}+x^{2}-8 x-3\right)\right) \\
& -6(x+1)^{3}\left(x^{2}-3 x+2\right) \operatorname{Li}_{2}(-x)\left(a\left(4 x^{4}+9 x^{3}+4 x^{2}+5 x+2\right)+v x\left(2 x^{3}+x^{2}-8 x-3\right)\right) \\
& -6(x-1)^{3}\left(x^{2}+3 x+2\right) \operatorname{Li}_{2}(x)\left(a\left(4 x^{4}-9 x^{3}+4 x^{2}-5 x+2\right)+v x\left(2 x^{3}-x^{2}-8 x+3\right)\right) \\
& \left.-24 x\left(a\left(x^{2}-4\right)-v\left(x^{4}-5 x^{2}+7\right)\right) \operatorname{ReLi}_{2}(x y)\right\} \\
& \delta_{T G, i}^{T}(x, v, a)=-\frac{1}{3}(v+a) \\
& \delta_{G 2}^{T}(x, v, a)=\frac{1}{72 \pi\left(x^{2}-4\right)\left(x^{2}-1\right)^{2}}\left\{+a^{2}\left(x^{2}-4\right)\left(4 x^{6}-15 x^{4}+126 x^{2}+3 \pi\left(20-3 x^{2}\right) \sqrt{4-x^{2}} x\right.\right. \\
& \left.+\pi^{2}\left(15 x^{3}-6 x^{2}+15 x-2\right)-115\right) \\
& +2 a v\left(57 x^{6}-330 x^{4}+453 x^{2}+3 \pi \sqrt{4-x^{2}}\left(x^{2}-2\right)^{2} x+\pi^{2}\left(5 x^{4}-2 x^{3}-25 x^{2}+6 x+10\right) x-180\right) \\
& -v^{2}\left(-4 x^{8}+31 x^{6}+6 x^{4}-341 x^{2}+3 \pi \sqrt{4-x^{2}}\left(3 x^{2}-16\right) x^{3}+\pi^{2}\left(5 x^{5}-2 x^{4}+5 x^{3}+10 x^{2}-80 x+8\right)+308\right) \\
& +6 \sin ^{-1}\left(\frac{x}{2}\right)\left(a^{2}\left(x^{2}-4\right)\left(3 x \sqrt{4-x^{2}}\left(3 x^{2}-20\right)+4 \pi\right)+2 a v\left(x^{2}-2\right)\left(4 \pi-3 x \sqrt{4-x^{2}}\left(x^{2}-2\right)\right)\right. \\
& \left.+v^{2} x^{2}\left(3 x \sqrt{4-x^{2}}\left(3 x^{2}-16\right)+4 \pi\right)\right)-24 \sin ^{-1}\left(\frac{x}{2}\right)^{2}\left(3 a^{2}\left(x^{2}-4\right)+2 a v\left(3 x^{2}-14\right)+v^{2}\left(3 x^{2}-16\right)\right) \\
& -6\left(x^{2}-4\right) \log (x)\left(a^{2}\left(9 x^{4}-26 x^{2}+2\right)+10 a v\left(x^{4}+2 x^{2}-2\right)+v^{2}\left(9 x^{4}-10 x^{2}-14\right)\right) \\
& -24(x-1)^{2}(x+2) \log (1-x) \log (x)\left(3 a^{2}(x-2) x+2 a v\left(x^{2}-2 x-2\right)-v^{2}\left(x^{2}-2 x+4\right)\right) \\
& +6(x+2) \log ^{2}(x)\left(a^{2}\left(3 x^{4}-24 x^{3}+39 x^{2}-2 x-8\right)+2 a v\left(x^{4}-8 x^{3}+11 x^{2}+6 x-10\right)\right. \\
& \left.-v^{2}\left(x^{4}-8 x^{3}+17 x^{2}-14 x+12\right)\right) \\
& +24(x-2)(x+1)^{2} \log (x) \log (x+1)\left(3 a^{2} x(x+2)+2 a v\left(x^{2}+2 x-2\right)-v^{2}\left(x^{2}+2 x+4\right)\right) \\
& +24(x-2)(x+1)^{2} \operatorname{Li}_{2}(-x)\left(3 a^{2} x(x+2)+2 a v\left(x^{2}+2 x-2\right)-v^{2}\left(x^{2}+2 x+4\right)\right) \\
& -12(x-1)^{2}(x+2) \operatorname{Li}_{2}(x)\left(3 a^{2}(x-2) x+2 a v\left(x^{2}-2 x-2\right)-v^{2}\left(x^{2}-2 x+4\right)\right) \\
& \left.-384 v(a+v) \operatorname{ReLi}_{2}(x y)\right\}
\end{aligned}
$$




$$
\begin{aligned}
\delta_{V G, r}^{F}(x, v, a)= & \frac{1}{36 \pi x \sqrt{4-x^{2}}\left(x^{2}-1\right)^{3}}\left\{+24 v x \sqrt{4-x^{2}}\left(x^{2}-1\right)^{3} \log \left(\frac{m_{t}^{2}}{\mu^{2}}\right)\right. \\
+ & x \sqrt{4-x^{2}}\left(a\left(2 \pi^{2}\left(2 x^{4}-6 x^{2}+3\right)-3(x-1)^{2}\left(11 x^{4}+18 x^{3}-18 x^{2}-22 x+3\right)\right)\right. \\
& \left.+v\left(2 \pi^{2}\left(6 x^{4}-2 x^{2}-5\right)-3(x-1)^{2}\left(19 x^{4}+34 x^{3}+22 x^{2}-38 x-45\right)\right)\right) \\
- & 120 x \sqrt{4-x^{2}}\left(x^{2}-2\right)(a+v) \sin ^{-1}\left(\frac{x}{2}\right)^{2}+48\left(x^{2}-1\right) \operatorname{ImLi}_{2}(y)\left(a\left(x^{2}-4\right)+v x^{2}\right) \\
+ & 48\left(x^{2}-1\right)\left(a\left(x^{2}-4\right)+v x^{2}\right) \operatorname{ImLi}_{2}((x-1) y)-48\left(x^{2}-1\right)\left(a\left(x^{2}-4\right)+v x^{2}\right) \operatorname{ImLi}_{2}(x y) \\
- & 24\left(x^{2}-1\right)\left(a\left(x^{2}-4\right)+v x^{2}\right) \operatorname{ImLi}_{2}\left(\frac{\left(x^{2}-1\right) y}{x}\right) \\
+ & 12 x \sqrt{4-x^{2}}\left(x^{2}-1\right) \log (2-x)\left(a\left(x^{4}-5 x^{2}+4\right)+v\left(x^{2}-3\right) x^{2}\right) \\
- & 12\left(x^{2}-1\right) \log (x)\left(a\left(x \sqrt{4-x^{2}}\left(x^{4}+3 x^{2}-4\right)-\pi\left(x^{2}-4\right)\right)+v\left(x \sqrt{4-x^{2}}\left(x^{2}+1\right)-\pi\right) x^{2}\right) \\
+ & 24 x^{3} \sqrt{4-x^{2}}\left(x^{2}-1\right)(a+v) \log ^{2}(x)+48 x \sqrt{4-x^{2}}\left(x^{2}-2\right)(a+v) \operatorname{ReLi}_{2}(x y) \\
+ & 12\left(x^{2}-1\right) \log (x+1)\left(a\left(x \sqrt{4-x^{2}}\left(x^{2}-1\right)^{2}-\pi\left(x^{2}-4\right)\right)+v x\left(\sqrt{4-x^{2}}\left(x^{4}+6 x^{2}-7\right)-\pi x\right)\right) \\
+ & \sin ^{-1}\left(\frac{x}{2}\right)\left(24\left(x^{2}-1\right) \log (x)\left(a\left(x^{2}-4\right)+v x^{2}\right)+72\left(x^{2}-1\right) \log (x+1)\left(a\left(x^{2}-4\right)+v x^{2}\right)\right. \\
& \left.+12\left(x^{4}-5 x^{2}+4\right)\left(a\left(x^{4}-3 x^{2}+4\right)+v\left(5-3 x^{2}\right) x^{2}\right)\right) \\
+ & \left.24 x \sqrt{4-x^{2}}\left(x^{2}-1\right) \operatorname{Li} 2(-x)\left(2 a x^{2}-5 a+6 v x^{2}+3 v\right)\right\} \\
& \quad \delta_{V G, i}^{F}(x, v, a)=-\frac{1}{3}(v+a)
\end{aligned}
$$

$$
\begin{aligned}
\delta_{T G, r}^{F}(x, v, a)= & -\frac{1}{18 \pi x\left(x^{2}-4\right)\left(x^{2}-1\right)^{3}}\left\{-12 v x\left(x^{2}-4\right)\left(x^{2}-1\right)^{3} \log \left(\frac{m_{t}^{2}}{\mu^{2}}\right)\right. \\
- & x\left(x^{2}-4\right)\left(a\left(x^{8}-2\left(5+\pi^{2}\right) x^{6}-20 x^{5}+63 x^{4}+8 x^{3}+\left(\pi^{2}-68\right) x^{2}+12 x+14\right)\right. \\
& \left.+v\left(\pi^{2}\left(4 x^{4}-x^{2}-4\right)+\left(2 x^{6}+4 x^{5}-29 x^{4}-66 x^{3}-25 x^{2}+68 x+58\right)(x-1)^{2}\right)\right) \\
+ & 60 x\left(x^{4}-6 x^{2}+8\right)(a+v) \sin ^{-1}\left(\frac{x}{2}\right)^{2}+48 \sqrt{4-x^{2}}\left(x^{2}-1\right) \operatorname{ImLi}_{2}(y)\left(v x^{2}-a\right) \\
+ & 48 \sqrt{4-x^{2}}\left(x^{2}-1\right)\left(v x^{2}-a\right) \operatorname{ImLi}_{2}((x-1) y)-48 \sqrt{4-x^{2}}\left(x^{2}-1\right)\left(v x^{2}-a\right) \operatorname{ImLi}_{2}(x y) \\
- & 24 \sqrt{4-x^{2}}\left(x^{2}-1\right)\left(v x^{2}-a\right) \operatorname{ImLi}_{2}\left(\frac{\left(x^{2}-1\right) y}{x}\right)+12 x^{3}\left(x^{2}-4\right)\left(x^{2}-1\right)^{2}(a+v) \log ^{2}(x) \\
- & 6 x\left(x^{4}-5 x^{2}+4\right) \log (2-x)\left(a\left(x^{4}-5 x^{2}+2\right)+v\left(x^{2}-2\right) x^{2}\right) \\
- & 6\left(x^{2}-1\right) \log (x)\left(a\left(x^{7}-9 x^{5}+22 x^{3}+2 \pi \sqrt{4-x^{2}}-8 x\right)+v\left(x^{5}-6 x^{3}-2 \pi \sqrt{4-x^{2}}+8 x\right) x^{2}\right) \\
- & 12\left(x^{2}-1\right) \log (x+1)\left(v x\left(2 x^{4}-10 x^{2}+\pi \sqrt{4-x^{2}} x+8\right)-a\left(2 x^{7}-10 x^{5}+8 x^{3}+\pi \sqrt{4-x^{2}}\right)\right) \\
+ & \sin ^{-1}\left(\frac{x}{2}\right)\left(24 \sqrt{4-x^{2}}\left(x^{2}-1\right) \log (x)\left(v x^{2}-a\right)+72 \sqrt{4-x^{2}}\left(x^{2}-1\right) \log (x+1)\left(v x^{2}-a\right)\right. \\
& \left.+6 \sqrt{4-x^{2}}\left(x^{4}-5 x^{2}+4\right)\left(a\left(x^{4}-3 x^{2}+4\right)+v\left(5-3 x^{2}\right) x^{2}\right)\right) \\
+ & 24 x\left(x^{4}-5 x^{2}+4\right) \operatorname{Li}_{2}(-x)\left(a\left(x^{4}+x^{2}+1\right)-v\left(2 x^{2}+1\right)\right) \\
- & \left.24 x\left(x^{4}-6 x^{2}+8\right)(a+v) \operatorname{ReLi}_{2}(x y)\right\}
\end{aligned}
$$

$$
\delta_{T G, i}^{F}(x, v, a)=-\frac{1}{3}(v+a)
$$




$$
\begin{aligned}
\delta_{G 2}^{F}(x, v, a)= & \frac{1}{72 \pi \sqrt{4-x^{2}}\left(x^{2}-1\right)^{2}}\left\{-96 x(a+v)^{2} \operatorname{ImLi}_{2}(y)+96 x(a+v)^{2} \operatorname{ImLi}_{2}(x y)\right. \\
+ & (x-1) \sqrt{4-x^{2}}\left(3 a^{2}\left(21 x^{3}-15 x^{2}+4\left(8 x^{2}-9 x-21\right) \log (2)-31 x+25\right)\right. \\
& +2 a v\left(4 x^{5}+4 x^{4}-17 x^{3}-13 x^{2}-49 x+12(3 x+11) \log (2)+71\right) \\
& \left.+3 v^{2}\left(21 x^{3}-15 x^{2}+4\left(8 x^{2}-x+3\right) \log (2)-31 x+25\right)\right) \\
- & 6 x^{2} \sqrt{4-x^{2}} \log (2-x)\left(a^{2}\left(x^{2}-2\right)-6 a v\left(x^{2}-6\right)+v^{2}\left(x^{2}-2\right)\right)-192 x(a+v)^{2} \log (x) \sin ^{-1}\left(\frac{x}{2}\right) \\
- & 6 \sqrt{4-x^{2}} \log (x)\left(a^{2}\left(7 x^{4}-16 x^{3}+x^{2}(20-8 \log (2))+24 x-36\right)+2 a v\left(3 x^{4}-8 x^{2} \log (2)-16 x+28\right)\right. \\
& \left.\left.+v^{2}\left(7 x^{4}-16 x^{3}+x^{2}(4-8 \log (2))-8 x+12\right)\right)+72 x^{2} \sqrt{4-x^{2}}(a+v)^{2} \log ^{2}(x)\right\} .
\end{aligned}
$$

The $\delta_{T, T G, G}^{\gamma}(\hat{x}, \hat{y})$ functions that appeared in Eq. (146) are:

$$
\begin{aligned}
& \delta_{T}^{\gamma}(\hat{x}, \hat{y})=\frac{1}{3 \pi}\left\{+2 \log \left(\frac{m_{t}^{2}}{\mu^{2}}\right)-6+\hat{y}-\frac{4 \pi^{2}}{3}+\frac{16(\hat{y}-1)}{\hat{x}^{2}}+\frac{4(\hat{y}-2)(\hat{y}-1)}{\hat{x}}-\frac{2(\hat{y}-1) \hat{y}(2 \hat{y}-1)}{\hat{x} \hat{y}-2}\right. \\
& +12 \sqrt{\frac{2}{\hat{x}}-1} \tan ^{-1}\left(\frac{1-\hat{y}}{\sqrt{\frac{2}{\hat{x}}-1}}\right)+\left(\hat{y}^{2}+2 \hat{y}-10\right) \log (1-\hat{y})-2 \log ^{2}(1-\hat{y}) \\
& -\frac{2}{\hat{x}^{3}}\left(\hat{x}^{2}-12 \hat{x}+16\right) \log \left(\frac{\hat{x}-2}{\hat{x} \hat{y}-2}\right)-6 \log \left(\frac{2-\hat{x}}{\hat{x}(\hat{y}-2) \hat{y}+2}\right)-\frac{\hat{x} \hat{y}(\hat{y}+2)+2}{\hat{x}} \log \left(\frac{\hat{x}(\hat{y}-2) \hat{y}+2}{2-\hat{x} \hat{y}}\right) \\
& \left.+4 \operatorname{Li}_{2}\left(-\frac{\hat{x}(\hat{y}-1)}{\hat{x}-2}\right)-2 \operatorname{Li}_{2}\left(\frac{\hat{x}(\hat{y}-1)^{2}}{\hat{x}-2}\right)\right\} \\
& \delta_{T G, r}^{\gamma}(\hat{x}, \hat{y})=\frac{2}{9 \pi}\left\{+4 \log \left(\frac{m_{t}^{2}}{\mu^{2}}\right)-11+\frac{5 \pi^{2}}{3}-\frac{(2-\hat{x})(1-\hat{y})\left(\hat{x}^{2} \hat{y}-2 \hat{x} \hat{y}-2 \hat{x}+8\right)}{\hat{x}^{2}(2-\hat{x} \hat{y})}\right. \\
& +4 \tan ^{-1}\left(\sqrt{\frac{2}{\hat{x}}-1}\right)^{2}-8\left(\sqrt{\frac{2}{\hat{x}}-1}-\tan ^{-1}\left(\sqrt{\frac{2}{\hat{x}}-1}\right)\right) \tan ^{-1}\left(\frac{1-\hat{y}}{\sqrt{\frac{2}{\hat{x}}-1}}\right) \\
& -\frac{4}{\hat{x}^{3}}\left(\hat{x}^{2}-4 \hat{x}+4\right) \log \left(\frac{2-\hat{x}}{2-\hat{x} \hat{y}}\right)-2 \log \left(\frac{\hat{x} \hat{y}^{2}}{2}\right) \log \left(\frac{1}{2}(2-\hat{x}(2-\hat{y}) \hat{y})\right)-4(1-\hat{y}) \log \left(\frac{2-\hat{x}(2-\hat{y}) \hat{y}}{(1-\hat{y})(2-\hat{x} \hat{y})}\right) \\
& \left.+2\left(-2 \operatorname{Li}_{2}\left(\frac{\hat{x} \hat{y}}{2}\right)+\operatorname{Li}_{2}\left(\frac{\hat{x}}{2}\right)-2 \operatorname{Li}_{2}(\hat{y})\right)-8 \operatorname{ReLi}_{2}\left(1-\frac{1}{2}(\hat{x}+i \sqrt{(2-\hat{x}) \hat{x}}) \hat{y}\right)\right\} \\
& \delta_{T G, i}^{\gamma}(\hat{x}, \hat{y})=-\frac{4}{9} \\
& \delta_{G}^{\gamma}(\hat{x}, \hat{y})=\frac{4}{27 \pi}\left\{+\frac{2 \pi^{2}}{3}-\frac{(2-\hat{x})(1-\hat{y})\left(3 \hat{x}^{2} \hat{y}-4 \hat{x} \hat{y}-8 \hat{x}+16\right)}{\hat{x}^{2}(2-\hat{x} \hat{y})}+\left(-2 \hat{x}+4 \log \left(\frac{\hat{x}}{2}\right)+4\right) \log (\hat{y})\right. \\
& +\frac{2}{\hat{x}^{3}}(2-\hat{x})\left(\hat{x}^{3}-\hat{x}^{2}+6 \hat{x}-8\right) \log \left(\frac{2-\hat{x}}{2-\hat{x} \hat{y}}\right)+(1-\hat{y})(3-\hat{y}) \log \left(\frac{\hat{x}(1-\hat{y})}{2-\hat{x} \hat{y}}\right) \\
& \left.+4\left(\operatorname{Li}_{2}\left(\frac{\hat{x} \hat{y}}{2}\right)-\mathrm{Li}_{2}\left(\frac{\hat{x}}{2}\right)-\mathrm{Li}_{2}(\hat{y})\right)\right\} \text {. }
\end{aligned}
$$

The $\delta_{S, S G, G 3}(x)$ functions that appeared in Eq. (148) are [ $\delta_{S}(x)$ also appeared in Eq. (176)]: 


$$
\begin{aligned}
\delta_{S}(x) & =\frac{1}{9 \pi x^{2}\left(1-x^{2}\right)}\left\{+36 x^{2}\left(x^{2}-1\right) \log \left(\frac{m_{t}}{\mu}\right)+\left(4 \pi^{2}-51\right) x^{2}\left(x^{2}-1\right)+6\left(5 x^{4}-7 x^{2}+2\right) \log (1-x)\right. \\
& -24 x^{4} \log (x)+24 x^{2}\left(x^{2}-1\right) \log (1-x) \log (x)+6\left(5 x^{4}-7 x^{2}+2\right) \log (x+1) \\
& \left.+24 x^{2}\left(x^{2}-1\right) \log (x) \log (x+1)+48 x^{2}\left(x^{2}-1\right) \operatorname{Li}_{2}(-x)+48 x^{2}\left(x^{2}-1\right) \operatorname{Li}_{2}(x)\right\} \\
\delta_{S G}(x) & =\frac{1}{9 \pi\left(x^{2}-1\right)^{2}}\left\{-36\left(x^{2}-1\right)^{2} \log \left(\frac{m_{t}}{\mu}\right)-6\left(\frac{\sqrt{4-x^{2}}}{x}\left(x^{4}-6 x^{2}+8\right)+2 \pi\right) \sin ^{-1}\left(\frac{x}{2}\right)\right. \\
& -36 \sin ^{-1}\left(\frac{x}{2}\right)^{2}-6 x^{2}\left(5 x^{2}+2\right) \log (x)-24 \log (1-x) \log (x)+12 \log ^{2}(x)-24 \log (x) \log (x+1) \\
& \left.-24 \operatorname{Li}_{2}(-x)-24 \operatorname{Li}_{2}(x)+24 \operatorname{ReLi}_{2}\left(\frac{\left(x^{2}-1\right) y}{x}\right)-3 \pi \sqrt{4-x^{2}}\left(x^{2}-2\right) x+3\left(x^{4}+8 x^{2}-9\right) x^{2}+5 \pi^{2}\right\} \\
& \\
& \delta_{G 3}(x)=-\frac{1}{36 \pi\left(1-x^{2}\right)^{2}}\left\{+271-620 x^{2}+342 x^{4}+8 x^{6}-x^{8}-156 \pi x \sqrt{4-x^{2}}+30 \pi x^{3} \sqrt{4-x^{2}}\right. \\
& \left.+36 x\left(26-5 x^{2}\right) \sqrt{4-x^{2}} \sin ^{-1}\left(\frac{x}{2}\right)-12\left(9 x^{4}+76 x^{2}-8\right) \log (x)\right\} .
\end{aligned}
$$

The $\delta_{S T}(x)$ and $\delta_{T T}^{(i)}$ functions that appeared in Eq. (176) are:

$$
\begin{aligned}
& \delta_{S T}(x)=-\frac{1}{9 \pi}\left[6 \log \left(\frac{m_{t}^{2}}{\mu^{2}}\right)+\frac{24\left(2-x^{2}\right) x^{2} \operatorname{Li}_{2}(-x)}{\left(1-x^{2}\right)^{2}}+24 \operatorname{Li}_{2}(x)-\frac{3\left(7 x^{2}+6 x+5\right)}{(x+1)^{2}}+\frac{2 \pi^{2}}{\left(1-x^{2}\right)^{2}}\right. \\
& \left.-\frac{6\left(3-x^{2}\right) \log (x+1)}{1-x^{2}}+18 \log (1-x)\right] \\
\delta_{T T}^{(1)}(x)= & -\frac{1}{9 \pi x^{2}\left(x^{2}-1\right)^{2}}\left[-6 x^{2}\left(x^{2}-1\right)^{2} \log \left(\frac{m_{t}^{2}}{\mu^{2}}\right)+12 x\left(4 x^{3}+7 x^{2}+2 x+3\right)(x-1)^{2} \operatorname{Li}_{2}(x)\right. \\
& +12 x(x+1)^{2}\left(4 x^{3}-7 x^{2}+2 x-3\right) \operatorname{Li}_{2}(-x)+6\left(x^{6}-3 x^{2}+2\right) \log (1-x)+6\left(x^{6}-3 x^{2}+2\right) \log (x+1)+x^{2} \\
& \left(3\left(x^{4}-22 x^{2}+21\right)+4 \pi^{2}\left(x^{4}-2 x^{2}-3\right)\right)-24 x^{2}\left(x^{4}-3 x^{2}-3\right) \log (x) \\
& \left.+12 x\left(2 x^{3}+3 x^{2}+3\right)(x-1)^{2} \log (1-x) \log (x)+12 x(x+1)^{2}\left(2 x^{3}-3 x^{2}-3\right) \log (x) \log (x+1)\right] \\
\delta_{T T}^{(2)}(x)= & -\frac{1}{9 \pi\left(x^{2}-1\right)^{2}}\left[-6\left(x^{2}-1\right)^{2} \log \left(\frac{m_{t}^{2}}{\mu^{2}}\right)-6(x+1)^{2}\left(x^{3}-10 x^{2}+13 x-8\right) \operatorname{Li}_{2}(-x)\right. \\
& +6(x-1)^{2}\left(x^{3}+10 x^{2}+13 x+8\right) \operatorname{Li}_{2}(x)-5 x^{6}+30 x^{4}+4 \pi^{2}\left(x^{4}+1\right)-9 x^{2}+18\left(x^{2}-1\right)^{2} \log (1-x) \\
& +18\left(x^{2}-1\right)^{2} \log (x+1)-12\left(5 x^{4}+x^{2}\right) \log (x)+6(x-1)^{2}\left(x^{3}+6 x^{2}+5 x+4\right) \log (1-x) \log (x) \\
& \left.+6\left(-x^{5}+4 x^{4}+6 x^{3}+3 x+4\right) \log (x) \log (x+1)-16\right]
\end{aligned}
$$

\section{Appendix B: Numerical results for flavor-changing decays}

The numerical values of $\Gamma_{i j}$ defined in Eq. (196) are given in Tables III-VI. In these tables we show the con- tributions from two-fermion operators, as well as their interferences with the $\mathrm{V}-\mathrm{V}$ four-fermion operators. To save space we do not include the operator $O_{\varphi q}^{(3,1+3)}$, whose contribution is the same as $O_{\varphi q}^{(1,1+3)}$ but with a differ- 
ent sign, and the operator $O_{l q}^{(3,1+3)}$, whose contribution is the same as $O_{l q}^{(1,1+3)}$. The percentage number under each entry represents the NLO correction (a dash implies the presented value vanishes at $\mathrm{LO}$ ).

We do not display $\Gamma_{i j}$ when both $i$ and $j$ correspond to $\mathrm{V}-\mathrm{V}, \mathrm{S}-\mathrm{S}$ and T-T operators. These contributions are simply

$$
\begin{aligned}
\Gamma_{t \rightarrow u e^{+} e^{-}}= & .9 \times 10^{-6} \mathrm{GeV}\left(\left|C_{l q}^{(1,1+3)}+C_{l q}^{(3,1+3)}\right|^{2}\right. \\
& \left.+\left|C_{q e}^{(1+3)}\right|^{2}+\left|C_{l u}^{(1+3)}\right|^{2}+\left|C_{e u}^{(1+3)}\right|^{2}\right) \\
& +8.2 \times 10^{-7} \mathrm{GeV}\left(\left|C_{\text {lequ }}^{(1,13)}\right|^{2}+\left|C_{\text {lequ }}^{(1,31)}\right|^{2}\right) \\
& +3.5 \times 10^{-5} \mathrm{GeV}\left(\left|C_{\text {lequ }}^{(3,13)}\right|^{2}+\left|C_{\text {lequ }}^{(3,31)}\right|^{2}\right) .
\end{aligned}
$$

The NLO corrections in these three terms are about $-8 \%$, $1 \%$ and $-8 \%$ respectively. The interference between $\mathrm{S}-\mathrm{S}$ and T-T operators is proportional to $\cos \theta$ and vanishes upon phase space integration. There is no other interference between any two four-fermion operators.
[1] Georges Aad et al. Observation of a new particle in the search for the Standard Model Higgs boson with the ATLAS detector at the LHC. Phys.Lett., B716:1-29, 2012.

[2] Serguei Chatrchyan et al. Observation of a new boson at a mass of $125 \mathrm{GeV}$ with the CMS experiment at the LHC. Phys.Lett., B716:30-61, 2012.

[3] Steven Weinberg. Phenomenological Lagrangians. Physica, A96:327, 1979.

[4] Steven Weinberg. Effective Gauge Theories. Phys.Lett., B91:51, 1980.

[5] H. Georgi. Effective field theory. Ann.Rev.Nucl.Part.Sci., 43:209-252, 1993.

[6] J.A. Aguilar-Saavedra. Single top quark production at LHC with anomalous Wtb couplings. Nucl.Phys., B804:160-192, 2008.

[7] J.A. Aguilar-Saavedra. Zt, gamma t and t production at hadron colliders via strong flavour-changing neutral couplings. Nucl.Phys., B837:122-136, 2010.

[8] Juan Antonio Aguilar-Saavedra. Effective operators in top physics. PoS, ICHEP2010:378, 2010.

[9] J.A. Aguilar-Saavedra. Effective four-fermion operators in top physics: A Roadmap. Nucl.Phys., B843:638-672, 2011.

[10] J.A. Aguilar-Saavedra, M.C.N. Fiolhais, and A. Onofre. Top Effective Operators at the ILC. JHEP, 1207:180, 2012.

[11] Ben Lillie, Jing Shu, and Timothy M.P. Tait. Top Compositeness at the Tevatron and LHC. JHEP, 0804:087, 2008.

[12] Kunal Kumar, Tim M.P. Tait, and Roberto VegaMorales. Manifestations of Top Compositeness at Colliders. JHEP, 0905:022, 2009.

[13] Bohdan Grzadkowski, Zenro Hioki, Kazumasa Ohkuma, and Jose Wudka. Probing anomalous top quark couplings induced by dimension-six operators at photon colliders. Nucl.Phys., B689:108-126, 2004.

[14] Bohdan Grzadkowski, Zenro Hioki, Kazumasa Ohkuma, and Jose Wfdka. Optimal beam polarizations for newphysics search through gamma gamma $\rightarrow \mathrm{t}$ anti- $\rightarrow$
lX/bX. JHEP, 0511:029, 2005.

[15] Bohdan Grzadkowski. Four Fermi effective operators at $e^{+} e^{-} \rightarrow \bar{t} t$. Acta Phys.Polon., B27:921-932, 1996.

[16] Bohdan Grzadkowski, Zenro Hioki, and Michal Szafranski. Four Fermi effective operators in top quark production and decay. Phys.Rev., D58:035002, 1998.

[17] Qing-Hong Cao and Jose Wudka. Search for new physics via single top production at $\mathrm{TeV}$ energy e gamma colliders. Phys.Rev., D74:094015, 2006.

[18] Qing-Hong Cao, Jose Wudka, and C.-P. Yuan. Search for new physics via single top production at the LHC. Phys.Lett., B658:50-56, 2007.

[19] K. Whisnant, Jin-Min Yang, Bing-Lin Young, and $\mathrm{X}$. Zhang. Dimension-six CP conserving operators of the third family quarks and their effects on collider observables. Phys.Rev., D56:467-478, 1997.

[20] Jin Min Yang and Bing-Lin Young. Dimension-six CP violating operators of the third family quarks and their effects at colliders. Phys.Rev., D56:5907-5918, 1997.

[21] Cen Zhang and Scott Willenbrock. Effective Field Theory for Top Quark Physics. Nuovo Cim., C033N4:285-291, 2010.

[22] Cen Zhang and Scott Willenbrock. Effective-FieldTheory Approach to Top-Quark Production and Decay. Phys.Rev., D83:034006, 2011.

[23] Nicolas Greiner, Scott Willenbrock, and Cen Zhang. Effective Field Theory for Nonstandard Top Quark Couplings. Phys.Lett., B704:218-222, 2011.

[24] Cen Zhang, Nicolas Greiner, and Scott Willenbrock. Constraints on Non-standard Top Quark Couplings. Phys.Rev., D86:014024, 2012.

[25] Scott Willenbrock. Effective Field Theory for Top and Weak Boson Physics. EPJ Web Conf., 28:05005, 2012.

[26] Celine Degrande, Jean-Marc Gerard, Christophe Grojean, Fabio Maltoni, and Geraldine Servant. Nonresonant New Physics in Top Pair Production at Hadron Colliders. JHEP, 1103:125, 2011.

[27] Celine Degrande, Jean-Marc Gerard, Christophe Grojean, Fabio Maltoni, and Geraldine Servant. An Effective 
approach to same sign top pair production at the LHC and the forward-backward asymmetry at the Tevatron. Phys.Lett., B703:306-309, 2011.

[28] C. Degrande, J.M. Gerard, C. Grojean, F. Maltoni, and G. Servant. Probing Top-Higgs Non-Standard Interactions at the LHC. JHEP, 1207:036, 2012.

[29] Jernej F. Kamenik, Michele Papucci, and Andreas Weiler. Constraining the dipole moments of the top quark. Phys.Rev., D85:071501, 2012.

[30] Ding Yu Shao, Chong Sheng Li, Jian Wang, Jun Gao, Hao Zhang, et al. Model independent analysis of top quark forward-backward asymmetry at the Tevatron up to $\mathcal{O}\left(\alpha_{s}^{2} / \Lambda^{2}\right)$. Phys.Rev., D84:054016, 2011.

[31] Jernej F. Kamenik, Jing Shu, and Jure Zupan. Review of New Physics Effects in t-tbar Production. Eur.Phys.J., C72:2102, 2012.

[32] P. Ko. Effective field theory for top physics. Nuovo Cim., C035N3:49-54, 2012.

[33] Celine Degrande. New physics signal in top physics. 2012.

[34] David Atwood, Sudhir Kumar Gupta, and Amarjit Soni. Same-sign Tops: A Powerful Diagnostic Test for Models of New Physics. JHEP, 1304:035, 2013.

[35] S.F. Taghavi and M. Mohammadi Najafabadi. Angular Correlations in Associated Production of Single Top and Higgs with and without anomalous $W t b$ Couplings. 2013.

[36] Zenro Hioki and Kazumasa Ohkuma. Probing nonstandard top-quark couplings via optimal-observable analyses at LHC. EPJ Web Conf., 49:17010, 2013.

[37] Miguel C.N. Fiolhais. Study of Top Effective Operators at the ILC. 2013.

[38] Sven Faller, Stefan Gadatsch, and Thomas Mannel. Minimal Flavour Violation and Anomalous Top Decays. Phys.Rev., D88:035006, 2013.

[39] Alper Hayreter and German Valencia. Constraints on anomalous color dipole operators from Higgs boson production at the LHC. Phys.Rev., D88:034033, 2013.

[40] Jean-Laurent Agram, Jeremy Andrea, Eric Conte, Benjamin Fuks, Denis Gel, et al. Probing top anomalous couplings at the LHC with trilepton signatures in the single top mode. Phys.Lett., B725:123-126, 2013.

[41] Werner Bernreuther and Zong-Guo Si. Top quark spin correlations and polarization at the LHC: standard model predictions and effects of anomalous top chromo moments. Phys.Lett., B725(1-3):115-122, 2013.

[42] Cen Zhang and Fabio Maltoni. Top-quark decay into Higgs boson and a light quark at next-to-leading order in QCD. Phys.Rev., D88:054005, 2013.

[43] M. Fabbrichesi, M. Pinamonti, and A. Tonero. Stringent limits on top-quark compositeness from top anti-top production at the Tevatron and the LHC. 2013.

[44] Antonio O. Bouzas and F. Larios. Probing $t t \gamma$ and $t t Z$ couplings at the LHeC. Phys.Rev., D88:094007, 2013.

[45] Jahred Adelman, Matthew Baumgart, Aran GarciaBellido, and Andrey Loginov. Determining Top Quark Couplings at the LHC: Snowmass White Paper. 2013.

[46] Tao Han and Richard Ruiz. Higgs from the Top. 2013.

[47] Harrison Mebane, Nicolas Greiner, Cen Zhang, and Scott Willenbrock. Effective Field Theory of Precision Electroweak Physics at One Loop. Phys.Lett., B724:259-263, 2013.

[48] Harrison Mebane, Nicolas Greiner, Cen Zhang, and Scott Willenbrock. Constraints on Electroweak Effective Operators at One Loop. Phys.Rev., D88(1):015028, 2013.

[49] Chien-Yi Chen, S. Dawson, and Cen Zhang. Electroweak
Effective Operators and Higgs Physics. Phys.Rev. D89:015016, 2014.

[50] J.A. Aguilar-Saavedra, J. Carvalho, Nuno Filipe Castro, Filipe Veloso, and A. Onofre. Probing anomalous Wtb couplings in top pair decays. Eur.Phys.J., C50:519-533, 2007.

[51] J.A. Aguilar-Saavedra. Top flavor-changing neutral interactions: Theoretical expectations and experimental detection. Acta Phys.Polon., B35:2695-2710, 2004.

[52] M. Fischer, S. Groote, J.G. Korner, and M.C. Mauser. Longitudinal, transverse plus and transverse minus $W$ bosons in unpolarized top quark decays at $\mathrm{O}(\operatorname{alpha}(s))$. Phys.Rev., D63:031501, 2001.

[53] Andrzej Czarnecki, Jurgen G. Korner, and Jan H. Piclum. Helicity fractions of $\mathrm{W}$ bosons from top quark decays at NNLO in QCD. Phys.Rev., D81:111503, 2010.

[54] M. Fischer, S. Groote, J.G. Korner, M.C. Mauser, and B. Lampe. Polarized top decay into polarized $\mathrm{W}: \mathrm{t}($ polarized $) \rightarrow \mathrm{W}$ (polarized $)+\mathrm{b}$ at $\mathrm{O}($ alpha-s $)$. Phys.Lett., B451:406-413, 1999.

[55] M. Fischer, S. Groote, J.G. Korner, and M.C. Mauser. Complete angular analysis of polarized top decay at O(alpha(s) ). Phys.Rev., D65:054036, 2002.

[56] Jure Drobnak, Svjetlana Fajfer, and Jernej F. Kamenik. New physics in $t \rightarrow b W$ decay at next-to-leading order in QCD. Phys.Rev., D82:114008, 2010.

[57] Jure Drobnak, Svjetlana Fajfer, and Jernej F. Kamenik. Flavor Changing Neutral Coupling Mediated Radiative Top Quark Decays at Next-to-Leading Order in QCD. Phys.Rev.Lett., 104:252001, 2010.

[58] Jure Drobnak, Svjetlana Fajfer, and Jernej F. Kamenik. QCD Corrections to Flavor Changing Neutral Coupling Mediated Rare Top Quark Decays. Phys.Rev., D82:073016, 2010.

[59] Jia Jun Zhang, Chong Sheng Li, Jun Gao, Hua Xing Zhu, C.-P. Yuan, et al. Next-to-leading order QCD corrections to the top quark decay via the FlavorChanging Neutral-Current operators with mixing effects. Phys.Rev., D82:073005, 2010.

[60] Chong Sheng Li and Tzu Chiang Yuan. QCD Correction to Charged Higgs Decay of the Top Quark. Phys.Rev., D42:3088, 1990.

[61] Zhe Dong, Gauthier Durieux, Jean-Marc Gerard, Tao Han, and Fabio Maltoni. Baryon number violation at the LHC: the top option. Phys.Rev., D85:016006, 2012.

[62] W. Buchmuller and D. Wyler. Effective Lagrangian Analysis of New Interactions and Flavor Conservation. Nucl.Phys., B268:621, 1986.

[63] B. Grzadkowski, M. Iskrzynski, M. Misiak, and J. Rosiek. Dimension-Six Terms in the Standard Model Lagrangian. JHEP, 1010:085, 2010.

[64] Aneesh Manohar and Howard Georgi. Chiral Quarks and the Nonrelativistic Quark Model. Nucl.Phys., B234:189, 1984.

[65] Elizabeth E. Jenkins, Aneesh V. Manohar, and Michael Trott. Renormalization Group Evolution of the Standard Model Dimension Six Operators I: Formalism and lambda Dependence. JHEP, 1310:087, 2013.

[66] J.A. Aguilar-Saavedra. A Minimal set of top-Higgs anomalous couplings. Nucl.Phys., B821:215-227, 2009.

[67] Gerard 't Hooft and M.J.G. Veltman. Regularization and Renormalization of Gauge Fields. Nucl.Phys., B44:189213, 1972.

[68] S.A. Larin. The Renormalization of the axial anomaly 
in dimensional regularization. Phys.Lett., B303:113-118, 1993.

[69] Patricia Ball and Roman Zwicky. $B_{D, S} \rightarrow \rho, \omega, K *, \phi$ decay form-factors from light-cone sum rules revisited. Phys.Rev., D71:014029, 2005.

[70] W. Beenakker, S. Dittmaier, M. Kramer, B. Plumper, M. Spira, et al. NLO QCD corrections to t anti-t $\mathrm{H}$ production in hadron collisions. Nucl.Phys., B653:151203, 2003.

[71] Elizabeth E. Jenkins, Aneesh V. Manohar, and Michael Trott. Renormalization Group Evolution of the Standard Model Dimension Six Operators II: Yukawa Dependence. JHEP, 1401:035, 2014.

[72] Rodrigo Alonso, Elizabeth E. Jenkins, Aneesh V. Manohar, and Michael Trott. Renormalization Group Evolution of the Standard Model Dimension Six Operators III: Gauge Coupling Dependence and Phenomenology. 2013.

[73] Peter L. Cho and Elizabeth H. Simmons. Searching for G3 in $t \bar{t}$ production. Phys.Rev., D51:2360-2370, 1995.

[74] G. Eilam, J.L. Hewett, and A. Soni. Rare decays of the top quark in the standard and two Higgs doublet models.
Phys.Rev., D44:1473-1484, 1991.

[75] B. Mele, S. Petrarca, and A. Soddu. A New evaluation of the $\mathrm{t} \rightarrow \mathrm{cH}$ decay width in the standard model. Phys.Lett., B435:401-406, 1998.

[76] J.A. Aguilar-Saavedra and B.M. Nobre. Rare top decays $\mathrm{t} \rightarrow \mathrm{c}$ gamma, $\mathrm{t} \rightarrow \mathrm{cg}$ and CKM unitarity. Phys.Lett., B553:251-260, 2003.

[77] K.G. Chetyrkin, Johann H. Kuhn, and M. Steinhauser. RunDec: A Mathematica package for running and decoupling of the strong coupling and quark masses. Comput.Phys.Commun., 133:43-65, 2000.

[78] J. Beringer et al. Review of Particle Physics (RPP). Phys.Rev., D86:010001, 2012.

[79] Bohdan Grzadkowski and Mikolaj Misiak. Anomalous Wtb coupling effects in the weak radiative B-meson decay. Phys.Rev., D78:077501, 2008.

[80] J.A. Aguilar-Saavedra, J. Carvalho, Nuno Filipe Castro, A. Onofre, and F. Veloso. ATLAS sensitivity to Wtb anomalous couplings in top quark decays. Eur.Phys.J., C53:689-699, 2008. 


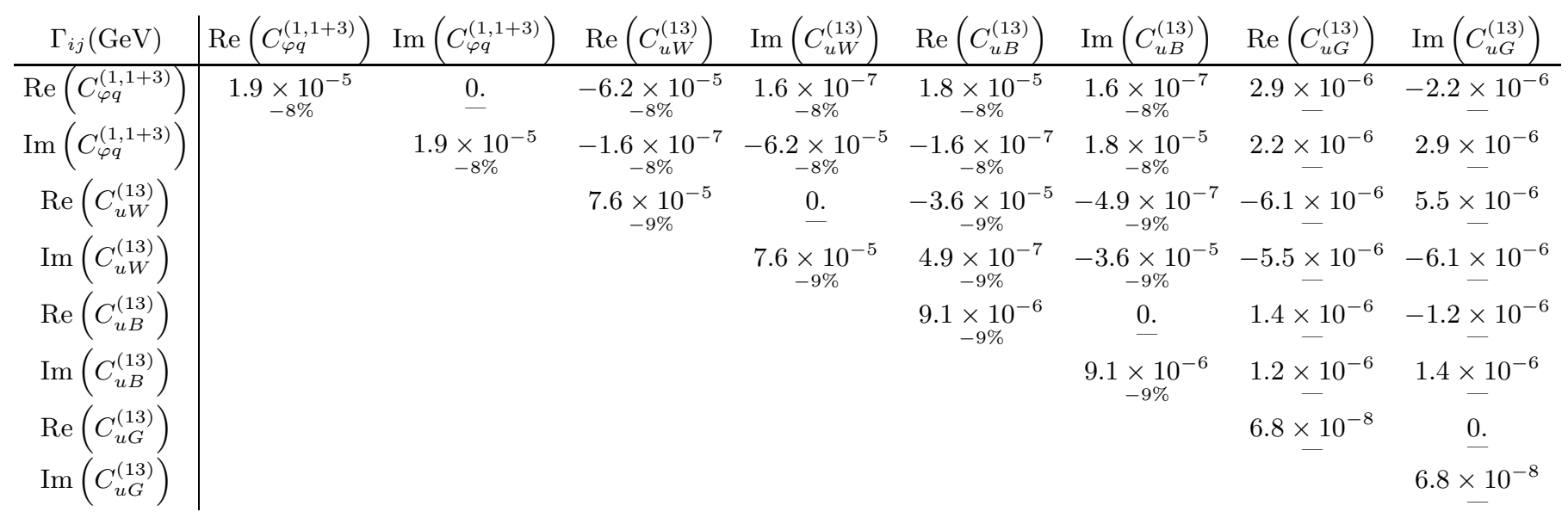

TABLE III: Contributions from two-fermion operators that involve a left-handed light quark.

\begin{tabular}{c|cccc}
$\Gamma_{i j}(\mathrm{GeV})$ & $\operatorname{Re}\left(C_{l q}^{(1,1+3)}\right)$ & $\operatorname{Im}\left(C_{l q}^{(1,1+3)}\right)$ & $\operatorname{Re}\left(C_{q e}^{(1+3)}\right)$ & $\operatorname{Im}\left(C_{q e}^{(1+3)}\right)$ \\
\hline $\operatorname{Re}\left(C_{\varphi q}^{(1,1+3)}\right)$ & $-3.5 \times 10^{-7}$ & $-2.3 \times 10^{-6}$ & $2.8 \times 10^{-7}$ & $1.8 \times 10^{-6}$ \\
$\operatorname{Im}\left(C_{\varphi q}^{(1,1+3)}\right)$ & $2.3 \times 10^{-6}$ & $-3.5 \times 10^{-7}$ & $-1.8 \times 10^{-6}$ & $2.8 \times 10^{-7}$ \\
$\operatorname{-in}$ & $-12 \%$ & $-8 \%$ & $-12 \%$ \\
$\operatorname{Re}\left(C_{u W}^{(13)}\right)$ & $-3.3 \times 10^{-6}$ & $3.8 \times 10^{-6}$ & $-1.4 \times 10^{-6}$ & $-3 . \times 10^{-6}$ \\
$\operatorname{Im}\left(C_{u W}^{(13)}\right)$ & $-3.8 \times 10^{-6}$ & $-3.3 \times 10^{-6}$ & $-7 . \times 10^{-6}$ & $-1.4 \times 10^{-6}$ \\
$\operatorname{Re}\left(C_{u B}^{(13)}\right)$ & $-1.9 \times 10^{-6}$ & $-1.1 \times 10^{-6}$ & $-2.5 \times 10^{-6}$ & $8.7 \times 10^{-7}$ \\
$\operatorname{Re}$ & $-7 \%$ & $-8 \%$ & $-7 \%$ & $-8 \%$ \\
$\operatorname{Im}\left(C_{u B}^{(13)}\right)$ & $1.1 \times 10^{-6}$ & $-1.9 \times 10^{-6}$ & $-8.7 \times 10^{-7}$ & $-2.5 \times 10^{-6}$ \\
$\operatorname{Re}\left(C_{u G}^{(13)}\right)$ & $2.6 \times 10^{-7}$ & $-2.5 \times 10^{-8}$ & $6.4 \times 10^{-9}$ & $2.3 \times 10^{-7}$ \\
$\operatorname{Im}\left(C_{u G}^{(13)}\right)$ & $2.5 \times 10^{-8}$ & $2.6 \times 10^{-7}$ & $-2.3 \times 10^{-7}$ & $6.4 \times 10^{-9}$ \\
& - & - & - & $-7 \%$
\end{tabular}

TABLE IV: Interference between two-fermion operators and V-V four-fermion operators that involve a left-handed light quark.

\begin{tabular}{|c|c|c|c|c|c|c|c|c|}
\hline$\Gamma_{i j}(\mathrm{GeV})$ & $\operatorname{Re}\left(C_{\varphi u}^{(1+3)}\right)$ & $\operatorname{Im}\left(C_{\varphi u}^{(1+3)}\right)$ & $\operatorname{Re}\left(C_{u W}^{(31)}\right)$ & $\operatorname{Im}\left(C_{u W}^{(31)}\right)$ & $\operatorname{Re}\left(C_{u B}^{(31)}\right)$ & $\operatorname{Im}\left(C_{u B}^{(31)}\right)$ & $\operatorname{Re}\left(C_{u G}^{(31)}\right)$ & $\operatorname{Im}\left(C_{u G}^{(31)}\right)$ \\
\hline $\operatorname{Re}\left(C_{\varphi u}^{(1+3)}\right)$ & $1.9 \times \underset{-8 \%}{10^{-5}}$ & 0. & $\begin{array}{c}-6.2 \times 10^{-5} \\
-8 \%\end{array}$ & $\begin{array}{c}-1.6 \times 10^{-7} \\
-8 \%\end{array}$ & $\begin{array}{c}1.8 \times 10^{-5} \\
-8 \%\end{array}$ & $\begin{array}{c}-1.6 \times 10^{-7} \\
-8 \%\end{array}$ & $-1.8 \times 10^{-6}$ & $-9.2 \times 10^{-7}$ \\
\hline $\operatorname{Im}\left(C_{\varphi u}^{(1+3)}\right)$ & & $\begin{array}{c}1.9 \times 10^{-5} \\
-8 \%\end{array}$ & $\begin{array}{c}-1.6 \times 10^{-7} \\
-8 \%\end{array}$ & 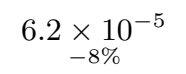 & $\begin{array}{c}-1.6 \times 10^{-7} \\
-8 \%\end{array}$ & $\begin{array}{c}-1.8 \times 10^{-5} \\
-8 \%\end{array}$ & $-9.2 \times 10^{-7}$ & $1.8 \times 10^{-6}$ \\
\hline $\operatorname{Re}\left(C_{u W}^{(31)}\right)$ & & & $\begin{array}{c}7.6 \times 10^{-5} \\
-9 \%\end{array}$ & 0 . & $\begin{array}{c}-3.6 \times 10^{-5} \\
-9 \%\end{array}$ & $\begin{array}{c}4.9 \times 10^{-7} \\
-9 \%\end{array}$ & $3.1 \times 10^{-6}$ & $1.9 \times 10^{-6}$ \\
\hline $\operatorname{Im}\left(C_{u W}^{(31)}\right)$ & & & & $\begin{array}{c}7.6 \times 10^{-5} \\
-9 \%\end{array}$ & $\begin{array}{c}-4.9 \times 10^{-7} \\
-9 \%\end{array}$ & $\begin{array}{c}-3.6 \times 10^{-5} \\
-9 \%\end{array}$ & $-1.9 \times 10^{-6}$ & $3.1 \times 10^{-6}$ \\
\hline $\operatorname{Re}\left(C_{u B}^{(31)}\right)$ & & & & & $\begin{array}{c}9.1 \times 10^{-6} \\
-9 \%\end{array}$ & 0. & $-1.3 \times 10^{-6}$ & $-9.6 \times 10^{-7}$ \\
\hline $\operatorname{Im}\left(C_{u B}^{(31)}\right)$ & & & & & & $\begin{array}{c}9.1 \times 10^{-6} \\
-9 \%\end{array}$ & $9.6 \times 10^{-7}$ & $-1.3 \times 10^{-6}$ \\
\hline $\operatorname{Re}\left(C_{u G}^{(31)}\right)$ & & & & & & & $5.3 \times 10^{-8}$ & 0 . \\
\hline $\operatorname{Im}\left(C_{u G}^{(31)}\right)$ & & & & & & & & $5.3 \times 10^{-8}$ \\
\hline
\end{tabular}

TABLE V: Contributions from two-fermion operators that involve a right-handed light quark. 


\begin{tabular}{c|cccc}
$\Gamma_{i j}(\mathrm{GeV})$ & $\operatorname{Re}\left(C_{l u}^{(1+3)}\right)$ & $\operatorname{Im}\left(C_{l u}^{(1+3)}\right)$ & $\operatorname{Re}\left(C_{e u}^{(1+3)}\right)$ & $\operatorname{Im}\left(C_{e u}^{(1+3)}\right)$ \\
\hline $\operatorname{Re}\left(C_{\varphi u}^{(1+3)}\right)$ & $-3.5 \times 10^{-7}$ & $-2.3 \times 10^{-6}$ & $2.8 \times 10^{-7}$ & $1.8 \times 10^{-6}$ \\
$-12 \%$ & $-8 \%$ & $-12 \%$ & $-8 \%$ \\
$\operatorname{Im}\left(C_{\varphi u}^{(1+3)}\right)$ & $2.3 \times 10^{-6}$ & $-3.5 \times 10^{-7}$ & $-1.8 \times 10^{-6}$ & $2.8 \times 10^{-7}$ \\
$-8 \%$ & $-12 \%$ & $-8 \%$ & $-12 \%$ \\
$\operatorname{Re}\left(C_{u W}^{(31)}\right)$ & $-3.3 \times 10^{-6}$ & $3.8 \times 10^{-6}$ & $-1.4 \times 10^{-6}$ & $-3 . \times 10^{-6}$ \\
$-7 \%$ & $-8 \%$ & $-7 \%$ & $-8 \%$ \\
$\operatorname{Im}\left(C_{u W}^{(31)}\right)$ & $3.8 \times 10^{-6}$ & $3.3 \times 10^{-6}$ & $-3 . \times 10^{-6}$ & $1.4 \times 10^{-6}$ \\
$-8 \%$ & $-7 \%$ & $-8 \%$ & $-7 \%$ \\
$\operatorname{Re}\left(C_{u B}^{(31)}\right)$ & $-1.9 \times 10^{-6}$ & $-1.1 \times 10^{-6}$ & $-2.5 \times 10^{-6}$ & $8.7 \times 10^{-7}$ \\
$-7 \%$ & $-8 \%$ & $-7 \%$ & $-8 \%$ \\
$\operatorname{Im}\left(C_{u B}^{(31)}\right)$ & $-1.1 \times 10^{-6}$ & $1.9 \times 10^{-6}$ & $8.7 \times 10^{-7}$ & $2.5 \times 10^{-6}$ \\
$\operatorname{Re}\left(C_{u G}^{(31)}\right)$ & $-8 \%$ & $-7 \%$ & $-8 \%$ & $-7 \%$ \\
$\operatorname{Re}$ & $7 . \times 10^{-8}$ & $2.1 \times 10^{-7}$ & $1.6 \times 10^{-7}$ & $4.3 \times 10^{-8}$ \\
$\operatorname{Im}\left(C_{u G}^{(31)}\right)$ & $2.1 \times 10^{-7}$ & $-6.8 \times 10^{-8}$ & $4.3 \times 10^{-8}$ & $-1.6 \times 10^{-7}$ \\
& - & - & - & -
\end{tabular}

TABLE VI: Interference between two-fermion operators and V-V four-fermion operators that involve a right-handed light quark. 